\title{
THE STRUCTURE OF A NONLINEAR ELLIPTIC OPERATOR
}

\author{
P. T. CHURCH, E. N. DANCER, AND J. G. TIMOURIAN
}

\begin{abstract}
Consider the nonlinear Dirichlet problem (1) $-\Delta u-\lambda u+u^{3}=g$, for $u: \Omega \rightarrow \mathbb{R}, u \mid \partial \Omega=0$, and $\Omega \subset \mathbb{R}^{n}$ connected and bounded, and let $\lambda_{i}$ be the $i$ th eigenvalue of $-\Delta u$ on $\Omega$ with $u \mid \partial \Omega=0,(i=1,2, \ldots)$. Define a map $A_{\lambda}: H \rightarrow H^{\prime}$ by $A_{\lambda}(u)=-\Delta u-\lambda u+u^{3}$, for either the Sobolev space $W_{0}^{1,2}(\Omega)=H=H^{\prime}$ (if $n \leq 4$ ) or the Hölder spaces $C_{0}^{2, \alpha}(\bar{\Omega})=H$ and $C^{0, \alpha}(\bar{\Omega})=H^{\prime}$ (if $\partial \Omega$ is $C^{2, \alpha}$ ), and define $A: H \times \mathbb{R} \rightarrow H^{\prime} \times \mathbb{R}$ by $A(u, \lambda)=\left(A_{\lambda}(u), \lambda\right)$. Let $G: \mathbb{R}^{2} \times E \rightarrow \mathbb{R}^{2} \times E$ be the global cusp map given by $G(s, t, v)=\left(s^{3}-t s, t, v\right)$, and let $F: \mathbb{R} \times E \rightarrow \mathbb{R} \times E$ be the global fold map given by $F(t, v)=\left(t^{2}, v\right)$, where $E$ is any Fréchet space.
\end{abstract}

Theorem 1. If $H=H^{\prime}=W_{0}^{1,2}(\Omega)$, assume in addition that $n \leq 3$. There exist $\varepsilon>0$ and homeomorphisms $\alpha$ and $\beta$ such that the following diagram commutes:

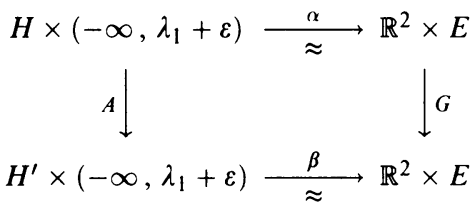

The analog for $A_{\lambda}$ with $\lambda_{1}<\lambda<\lambda_{1}+\varepsilon$ is also given. In a very strong sense this theorem is a perturbation result for the problem (1): As $g$ (and $\lambda$ ) are perturbed, it shows how the number of solutions $u$ of (1) varies; in particular, that number is always 1,2 or 3 for $\lambda<\lambda_{1}+\varepsilon$.

A point $u \in H$ is a fold point of $A$ if the germ of $A$ at $u$ is $C^{0}$ equivalent to the germ of $F$ at $(0,0)$ (i.e. under homeomorphic coordinate changes in domain near $u$ and in range near $A(u), A$ becomes $F$ ), and the singular set $S A$ is the set of points at which $A$ fails to be a local diffeomorphism. For larger values of $\lambda$ our information is limited:

Theorem 2. Consider the Sobolev case with $n \leq 4$ and $\partial \Omega C^{\infty}$. For all $\lambda \in \mathbb{R}$, (i) $\operatorname{int}(S A)=\varnothing$; (ii) there is a dense subset $\Gamma$ in $S A$ of fold points, and (iii) for $\lambda<\lambda_{2}, S A$ [resp., for $n \leq 3$ and $\lambda<\lambda_{2}, S A-\Gamma$ ] is a real analytic submanifold of codimension 1 in $H \times \mathbb{R}$ [resp., $S A]$.

Received by the editors November 8, 1989 and, in revised form, April 10, 1991.

1991 Mathematics Subject Classification. Primary 58C27, 35J65; Secondary 47H15.

Key words and phrases. Nonlinear partial differential equations, elliptic boundary value problem, nonlinear Dirichlet problem, singularity theory in infinite dimensions, fold map, cusp map.

The authors were partially supported by NSERC Contract A7357. Church is grateful to the University of Alberta for its hospitality during 1986-87 and the summers of 1988 and 1989. Dancer thanks the University of Alberta for its hospitality and support during a visit in 1986. 


\section{INTRODUCTION}

Consider the nonlinear boundary value problem

$$
-\Delta u-\lambda u+u^{3}=g \quad \text { on } \Omega, \quad u \mid \partial \Omega=0,
$$

where $\Omega \subset \mathbb{R}^{n}$ is a bounded open connected subset. For $n \leq 4$ and the Sobolev space $W_{0}^{1,2}=H=H^{\prime}$ define $A_{\lambda}: H \rightarrow H^{\prime}$ by

$$
\left\langle A_{\lambda}(u), \phi\right\rangle_{H}=\int_{\Omega}\left[\nabla u \nabla \phi-\lambda u \phi+u^{3} \phi\right]
$$

for each $\phi \in C_{0}^{\infty}(\Omega)$, i.e. $A_{\lambda}(u)=-\Delta u-\lambda u+u^{3}$ in the weak sense, and define $A: H \times \mathbb{R} \rightarrow H^{\prime} \times \mathbb{R}$ by $A(u, \lambda)=\left(A_{\lambda}(u), \lambda\right)$. For the Hölder spaces (1.8) $C_{0}^{2, \alpha}(\bar{\Omega})=H$ and $C^{0, \alpha}(\bar{\Omega})=H^{\prime}$ of $C^{k, \alpha}(\Omega)(k=0,2)$ functions 0 (if there is a subscript 0 ) on $\partial \Omega$, where $\partial \Omega$ is a $C^{2, \alpha}$ manifold and $n$ is arbitrary, define $A_{\lambda}: H \rightarrow H^{\prime}$ by

$$
A_{\lambda}(u)=-\Delta u-\lambda u+u^{3},
$$

and again $A(u, \lambda)=\left(A_{\lambda}(u), \lambda\right)$.

Our goal is to determine for each $g$ and $\lambda$ the number of solutions $u$ of the given boundary value problem (1), and how that number changes as $g$ and $\lambda$ are perturbed. The number of solutions corresponds to the number of point inverses $A^{-1}(g, \lambda)=\left(A_{\lambda}^{-1}(g), \lambda\right)$ of the map $A$.

For any Fréchet space $E$ define the global cusp map

$$
G=w \times \mathrm{id}: \mathbb{R}^{2} \times E \rightarrow \mathbb{R}^{2} \times E, \quad(s, t, v) \mapsto\left(s^{3}-t s, t, v\right) ;
$$

$G$ is an elementary catastrophe on Thom's list [WP and GG, p. 147], and for $E=\{0\}$ was defined by Hassler Whitney. Note that $s^{3}-t s=g$ has a unique solution $s$ for each fixed $t \leq 0$ and $g$, while for each $t>0$, there is an open interval $J$ of values $g$ each of which has three solutions $s$.

Let $\lambda_{i}$ be the $i$ th eigenvalue (counting multiplicity) of $-\Delta$ on $\Omega$ with null boundary conditions, $\left(\lambda_{1}<\lambda_{2} \leq \lambda_{3} \leq \ldots\right)$. The following theorem given in (8.3) and (8.5) is the main result of this paper. We assume $n \leq 3$ in the Sobolev case and $\partial \Omega$ is $C^{2, \alpha}$ in the Hölder case.

1.1. Theorem. (a) There exist $\varepsilon>0$ and homeomorphisms $\alpha$ and $\beta$ such that the following diagram commutes:

$$
\begin{array}{ccc}
H \times\left(-\infty, \lambda_{1}+\varepsilon\right) & \stackrel{\alpha}{\longrightarrow} \mathbb{R}^{2} \times E \\
A \downarrow & & \downarrow G \\
H^{\prime} \times\left(-\infty, \lambda_{1}+\varepsilon\right) & \stackrel{\beta}{\approx} \mathbb{R}^{2} \times E
\end{array}
$$

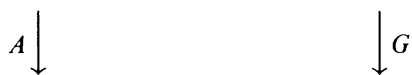

(b) Moreover, for $\lambda_{1}<\lambda<\lambda_{1}+\varepsilon$ there are homeomorphisms $\phi$ : $H \approx \mathbb{R}^{2} \times E$ and $\psi: H \approx \mathbb{R}^{2} \times E$ such that $\psi A_{\lambda}=G \phi$.

Part (b) is probably of more interest to researchers in partial differential equations. Under the given restriction on $\lambda$, it says that the nonlinear partial differential operator $A_{\lambda}$ is-up to nonlinear global changes of coordinates in the domain and target spaces-globally the same as the cusp map $G$. Thus the 
cusp map serves as a normal form for $A_{\lambda}$. This seems to be the first example of a partial differential operator proved globally equivalent to the cusp map $G$. (In [Ma] Mandhyan proves that a given integral operator is globally equivalent to $G$.)

Proving this equivalence yields rather complete information about the nonlinear elliptic boundary value problem (1). It implies that for all $g$ of suitable smoothness, problem (1) has exactly 1,2 or 3 solutions (with all possibilities occurring depending on $g$ ). In particular, for "generic" $g$ there are exactly 1 or 3 solutions. All this information is new. In addition to giving exact multiplicity results for problem (1), we expect that the general approach of this paper may be applied to other problems, and some results in this direction are given in [CT-2].

In independent work Bernhard Ruf [R-2] treats the Neumann version of the boundary value problem (1) in the Hölder case, and obtains multiplicity results and other properties of the global cusp map $G$. This version is somewhat simpler than the Dirichlet case (e.g. the first eigenfunction of $-\Delta$ is constant), and he obtains an explicit value for $\varepsilon$, viz. $\lambda_{2} / 12$. He does not prove that his map is globally equivalent to $G$, i.e. the same as $G$ after global coordinate changes. In the last section he states that his arguments may be altered to apply to the Dirichlet version for some domains. (Note that the hypotheses of [R-2, Proposition 6.1] do not apply to all smooth bounded domains $\Omega$.) In contrast, our results are for the Dirichlet problem (1) and treat both the Hölder case for all $C^{2, \alpha}$ bounded domains $\Omega$ and the Sobolev case with $n \leq 3$ for all bounded domains. We do not identify $\varepsilon$ explicitly, but we take the step of proving global equivalence of $A_{\lambda}$ to the cusp map $G$.

In [R-1, Corollary 22 and Theorem 22] Ruf proves that for certain domains, e.g., $\Omega=(0,1)$, in the Hölder case there are $\lambda<\lambda_{2}$ for which $A_{\lambda}$ has swallowtail and butterfly singularities, and thus there are $g$ for which problem (1) has at least five solutions. He states that similar arguments work for the Dirichlet case. This implies that our Theorem 1.1 is sharp in the sense that $\lambda<\lambda_{1}+\varepsilon$ for some $\varepsilon>0$ cannot be replaced by $\lambda<\lambda_{2}$.

The present paper uses a variety of analysis together with singularity theory and some topology to identify $A_{\lambda}$ and $A$ up to coordinate change, and thereby answer multiplicity and perturbation questions. This general approach began with a 1972 paper [AP] of Ambrosetti and Prodi. They consider a particular nonlinear Dirichlet problem, study the corresponding operator $\Phi: C_{0}^{2, \alpha}(\bar{\Omega}) \rightarrow$ $C^{0, \alpha}(\bar{\Omega})$ of Hölder spaces, and give interesting information about its structure and point inverses (number of solutions). Berger and Podolak [BP] in 1975 consider the corresponding operator in Sobolev spaces $W_{0}^{1,2}(\Omega)$ and obtain analogous conclusions. In 1979 Berger and Church [BC-1] (see also [BC-2]) show that the Berger-Podolak operator is-after coordinate changes in domain and target spaces-globally the map $F: \mathbb{R} \times E \rightarrow \mathbb{R} \times E$ ( $E$ is a Banach space) defined by $F(t, v)=\left(t^{2}, v\right)$. This seems to be the first instance in which a nonlinear operator arising from a boundary value problem is shown equivalent (in this sense) to a specific map not a homeomorphism.

McKean and Scovel [MS] in 1986 study two boundary value problems in ordinary differential equations and show that one of the operators is globally equivalent to the fold map $F$. In 1989 Mandhyan [Ma] shows that a certain 
integral operator is equivalent to $F$. Recently [CT-3] Church and Timourian give a global abstract characterization of $F$, and apply it to all the above examples. The resulting proofs are shorter, often with stronger conclusions, than the originals, and this characterization should be useful in studying other problems.

The paper [AP] inspired much work on the existence of solutions where the limits of the nonlinearity cross the eigenvalues of $-\Delta$ suitably (e.g. Lazer and McKenna [LMc] and their references). Berger's recent book [B-1] includes a discussion of some general principles for normal form theorems, together with consideration of various examples. Cafagna [Ca] gives a survey of the use of singularity theory in boundary value problems.

Local cusp singularities (i.e. maps locally the same as $G$ ) arise in the work of Cafagna and Donati [CD-1 and CD-2], Cafagna and Tarantello [CaT], Cafagna [Ca], Lazari and Micheletti [LM], Mandhyan [Ma], and of course Ruf [R-1 and R-2], as well as the predecessors [I, II, III, CT-1 and CT-2] of the present paper. A series of papers by McKenna and others culminating in [CJM] discusses oscillation in a suspension bridge and gives evidence of cusp like behavior. In a 1987 paper McKean [Mc] shows that a differential operator arising from a boundary value problem in [MS] has all the (local) Morin singularities in the equidimensional case displayed transversally [GG, p. 177], and, as noted above, Ruf [R-2] also find higher order singularities. Singularity theory is also extensively applied in differential equations in the work of Golubitsky, Schaeffer and Stewart [GS and GSS] and that of Damon [D].

Our perspective here, however, is global, not local: We wish to identify the differential operators $A$ and $A_{\lambda}$ up to global coordinate change. In [II] Berger, Church and Timourian proved the analog of (1.1) for $A, \lambda<\lambda_{1}+\varepsilon$ and $\|u\|_{H}<$ $\varepsilon$ in the Sobolev case; the present paper removes the restriction on $\|u\|_{H}$. In [CT-2] Church and Timourian give two abstract global characterizations of the cusp map $G$, restated here as (8.1) and (8.2), and they are applied here to prove the desired equivalence. They are also applied in [CT-2] to give another proof that the Mandhyan integral operator is globally equivalent to $G$, and are partially applied to boundary value problems in [CD-2, Ca and R-2].

Because this paper, like its predecessors, is aimed at researchers in singularity theory as well as those in partial differential equations, we have included more detailed referencing than is customary.

1.2. Definition. Let $X$ and $Y$ be $C^{k}(k=0,1,2, \ldots$ or $\infty)$ manifolds modeled on real Banach spaces (Fréchet spaces if $k=0$ ), and let $\Phi: X \rightarrow Y$ be a $C^{k}$ map. The singular set $S \Phi$ is the set of all $x \in X$ at which $\Phi$ fails to be a local $C^{k}$ diffeomorphism. (A $C^{0}$ diffeomorphism is a homeomorphism. Note that $S \Phi$ for $k \geq 1$ differs from that for $k=0$.)

1.3. Definition. Two $C^{k}$ maps $\Phi: X \rightarrow Y$ and $\Upsilon: X^{\prime} \rightarrow Y$ are called equivalent at $x \in X \cap X^{\prime}$ if there exists $U$ an open neighborhood of $x$ in $X$ and in $X^{\prime}$ such that the restriction maps $\Phi|U=\Upsilon| U$; the equivalence class at $x$ of $\Phi$ is called the germ of $\Phi$ at $x$.

1.4. Definition. Two $C^{k}(k=0,1, \ldots$ or $\propto)$ maps $\Phi: X \rightarrow Y$ and $\Psi: X^{\prime}$ $\rightarrow Y^{\prime}$ are called $C^{k}$ equivalent if there exist $C^{k}$ diffeomorphisms $\alpha: X \rightarrow X^{\prime}$ and $\beta: Y \rightarrow Y^{\prime}$ such that $\Psi \alpha=\beta \Phi$. Two $C^{k}$ map germs $\Phi$ at $x$ and $\Psi$ at 
$x^{\prime}$ are $C^{k}$ equivalent if there exist $C^{k}$ diffeomorphism germs $\alpha$ at $x$ and $\beta$ at $\Phi(x)$ such that $\alpha(x)=x^{\prime}$ and $\Psi \alpha=\beta \Phi$.

1.5. Definition. For a Fréchet space $E$ the global fold map $F: \mathbb{R} \times E \rightarrow \mathbb{R} \times E$ is defined by $F(t, v)=\left(t^{2}, v\right)$. The germ of a map $\Phi$ at $x$ is a $\left(C^{k}\right)$ fold [resp., cusp] if it is $C^{k}$ equivalent to the germ of $F$ [resp., $G$ ] at $(0,0)$ [resp., $(0,0,0)$ ], and $x$ is called a fold [resp., cusp] point of the map $\Phi$. A topological fold [resp., cusp] is a $C^{0}$ fold [resp., cusp]. Abstract (local) characterizations of fold at $x$ and cusp at $x$ are given in [I, Theorem 1.5, p. 2 and Theorem 1.7, p. 3]. Abstract characterizations of the global fold and the global cusp are given in [CT-2, (3.8), (2.2) and (2.7)], see (8.1) and (8.2). The set of cusp points is denoted by $C A$.

The following theorem is an amalgamation of the results of (4.1), (4.3), (4.6) and (4.8).

\subsection{Theorem. Consider the map A resulting from equation}

$$
-\Delta u-\lambda u+u^{3}=g \quad \text { on } \Omega, \quad u \mid \partial \Omega=0,
$$

in the Sobolev case (see equation (2)) with $\Omega$ bounded in $\mathbb{R}^{n}, n \leq 4$ and $\partial \Omega C^{\infty}$. Then:

(a) The interior of $S A$ in $H \times \mathbb{R}$ is empty, i.e. $S A$ is meager.

(b) There is an open dense subset $\Gamma \subset S A$ such that every $(u, \lambda) \in \Gamma$ is a fold point of $A$ (and $u$ is a fold point of $A_{\lambda}$ ).

(c) For every nonempty connected open subset $V \subset H \times \mathbb{R}, V-(S A-\Gamma)$ is nonempty connected open, i.e. $S A-\Gamma$ is supermeager in $H \times \mathbb{R}[\mathrm{CT}-1$, (1.5)].

(d) Here $\partial \Omega$ need not be $C^{\infty}$. The singular set $S A \cap\left(H \times\left(-\infty, \lambda_{2}\right)\right)$ is a real analytic manifold of codimension one in $H \times\left(-\infty, \lambda_{2}\right)$, and for $n \leq 3,(S A-\Gamma) \cap\left(H \times\left(-\infty, \lambda_{2}\right)\right)$ is a $C^{\infty}$ manifold of codimension one in $S A \cap\left(H \times\left(-\infty, \lambda_{2}\right)\right)$.

While Theorem 1.1 gives much more information in case $\lambda<\lambda_{1}+\varepsilon$ for $\varepsilon>0$ sufficiently small, Theorem $1.6(\mathrm{a})(\mathrm{b})(\mathrm{c})$ [resp., (d)] applies to all real $\lambda$ [resp., all $\lambda<\lambda_{2}$ ].

1.7. Outline of the paper. To a large extent the paper is a proof (see (8.3)) of (1.1) (a); it involves verifying each of the conditions of the abstract characterization of the global cusp map (8.1) (from [CT-2]). Let $\bar{A}$ refer to

$$
A: H \times\left(-\infty, \lambda_{1}+\varepsilon\right) \rightarrow H \times\left(-\infty, \lambda_{1}+\varepsilon\right)
$$

for $\varepsilon>0$ sufficiently small. Condition (i) of (8.1) is immediate, and (v) follows from [III] (see (8.3)). In $\S 2$ we prove that (ii) $A$ is proper, and in $\S 5$ we show that (iii) $S \bar{A}$ has only folds and (positive) cusps. In $\S 6$ we give (iv) the injectivity of $\bar{A}$, and in $\S 7$ we show that (vi) $(S \bar{A}, C \bar{A})$ is homeomorphic to $(H, \Lambda)$, where $\Lambda$ is a codimension one subspace of $H$. Examples are given in [CT-2] to show the necessity of each of the conditions of (8.1) and (8.2), so we cannot omit any of them.

The analog (1.1)(b) for $A_{\lambda}$ uses, in addition, $\S 3$ on $A_{\lambda}$ (especially (3.9) and (3.13) that $S A_{\lambda} \approx H$ ) and the latter part of $\S 7\left(C A_{\lambda}\right.$ is contractible). Theorem (1.6) is given in $\S 4$. 
1.8. Notation. The symbol $C^{k, \alpha}(\bar{\Omega})(k=0,2)$ with $C^{\alpha}=C^{0, \alpha}$ refers to the space of functions whose $k$ th order partials are uniformly Hölder continuous on $\Omega$, [GT, p. 52] and $C_{0}^{2, \alpha}(\bar{\Omega})=\left\{u \in C^{2, \alpha}(\bar{\Omega}): u \mid \partial \Omega=0\right\}$. See also [Z, pp. 230-233 and pp. 248-249]. The symbol $\phi_{i}(i=1,2, \ldots)$ is the $i$ th eigenfunction of $-\Delta$ with null boundary conditions, normalized with $\left\|\phi_{i}\right\|_{2}=1$ unless otherwise specified.

1.9. Conventions. Except for those sections specifically dealing with Hölder spaces ( $\S 2$ and (A.3)), proofs are given in the Sobolev case, with natural substitutions made for the Hölder case. Thus for $A_{\lambda}: H \rightarrow H$, the domain $H$ is interpreted as $C_{0}^{2, \alpha}(\bar{\Omega})$, the range $H$ is $C^{0, \alpha}(\bar{\Omega})$, and the $H$-inner product $\langle,\rangle_{H}$ becomes the $L^{2}(\Omega)$-inner product.

Unless explicitly stated otherwise, all lemmas and propositions are given for

$$
-\Delta u-\lambda u+f(u)=g, \quad u \in \Omega, \quad u \mid \partial \Omega=0,
$$

with $f$ and $\Omega$ satisfying hypotheses (1.10) below and they apply both to the Sobolev and Hölder cases. In $\S 2$ some results are stated for explicit hypotheses which are a part of (1.10); thus (1.10) is certainly adequate. A few results explicitly state Sobolev case or $f(u)=u^{3}$, to indicate a restriction. See (1.11).

Many results are cited from [II and III], and versions of these results for problem (5) are discussed in the Appendix, (A.2) and (A.3). Thus a citation of [II] or [III] may implicitly involve a reference to (A.2) and (A.3). In the Appendix we do not assume hypotheses (1.10).

The first eigenfunction of problem (5) is denoted by $e$ and is taken to be positive on $\Omega$ [III, (1.6)] with $\|e\|_{2}=1$ unless otherwise specified. (In (7.1) $e$ is chosen to have $\|e\|_{H}=1$.)

\subsection{Hypotheses. Assume $\Omega$ is a bounded connected open set in $R^{n}$.}

(1) In the Sobolev version $\left(A_{\lambda}: W_{0}^{1,2}(\Omega) \rightarrow W_{0}^{1,2}(\Omega)\right)$ assume:

(i) $n \leq 3, f: \mathbb{R} \rightarrow \mathbb{R}$ is $C^{k}(k \geq 3)$, and $f^{(3)} \in L^{\infty}(\mathbb{R})$;

(ii) $0=f(0)=f^{\prime}(0)=f^{\prime \prime}(0)$ and $f^{(3)}(s)>0$ for all $s \in \mathbb{R}$.

Hypotheses (ii') are: $f^{\prime}(s) \geq 0$ and $f^{(3)}(s) \neq 0$ for all real $s, f^{\prime \prime}(s) \neq 0$ for all real nonzero $s$ and $f^{\prime}(0)=0=f(0)$. Then (ii') implies (A.1) that $f^{\prime}(s)>0$ and $s f^{\prime \prime}(s)>0$ for $s \neq 0, f^{(3)}(s)>0$ for all $s$ and $f^{\prime \prime}(0)=0$, and one may verify that ii) and $\left.\mathrm{ii}^{\prime}\right)$ are equivalent and $f^{(j)}(s) s^{3-j}>0$ for $s \neq 0(j=0,1,2,3)$. Often we will add another hypothesis:

(iii) There exists $\eta>0$ with $\eta<f^{(3)}(s)$ for all $s \in \mathbb{R}$.

Thus

$$
(\eta /(3-j) !)|s|^{3-j}<\left|f^{(j)}(s)\right| \quad(s \in \mathbb{R} ; j=0,1,2,3) .
$$

(2) In the Hölder version $\left(A_{\lambda}: C_{0}^{2, \alpha}(\bar{\Omega}) \rightarrow C^{\alpha}(\bar{\Omega})\right)$ assume:

(i) $n$ is arbitrary, $\partial \Omega$ is $C^{2, \alpha}$, and $f: \mathbb{R} \rightarrow \mathbb{R}$ is $C^{k+1}(k \geq 2)$;

(ii) and (iii) as above.

Note that if $f(u)=u^{3}, \Omega \subset \mathbb{R}^{n}$ is bounded, and $n \leq 3$ in the Sobolev case and $\Omega$ is $C^{2, \alpha}$ in the Hölder case, then the above hypotheses are satisfied.

1.11. Remark. Theorem $(1.1)(\mathrm{a})$ and (b) is actually proved $((8.3)$ and $(8.5))$ for $f(u)=u^{3}$ replaced by $(*)$ any $f$ satisfying $(1.10)$ with $\left\|f^{(3)}\right\|_{\infty}<x$. 
In both $(1.1)(a)$ and (b) the proof is the verification of the conditions of the abstract characterization of $G$ given in (8.1) and (8.2). All these conditions are verified in the generality $(*)$.

\section{The MAP $A$ is A PROPER Fredholm MAP OF INDEX 0}

We suppose hypotheses (1.10). In the Sobolev case, $A$ is Fredholm of index 0 by [II, (2.4)]. The proof of the properness of $A$ in [II, (2.9)] used somewhat different hypotheses than (1.10), and in (2.1) we prove properness assuming (1.10). In the rest of this section we prove the analogous results in the Hölder case. Where the Hölder results do not require all of (1.10), the lesser hypotheses are indicated; (1.10) always suffices.

2.1. Lemma. Sobolev case. The map $A$ is proper.

Proof. We first prove under hypotheses (1.10) that $N$ is compact, where

$$
\langle N(u), \phi\rangle_{H}=\int_{\Omega} f(u) \phi
$$

for every $\phi \in C_{0}^{\infty}(\Omega)$. Suppose $\left\|u_{m}\right\|_{H} \leq K$, where $K>1$. There is a subsequence $u_{m_{k}} \rightarrow u$ in $L^{4}(\Omega)$ [A, p. 147], and it follows from (1.10) and Taylor's formula at 0 [Z, pp. 148-149] that

$$
\left|f^{(j)}(s)\right| \leq\left(\left\|f^{(3)}\right\|_{\infty} /(3-j) !\right)|s|^{3-j} \quad(s \in \mathbb{R} ; j=0,1,2,3) .
$$

From Taylor's formula at $u$ and Hölder's Inequality [B-2, p. 28],

$$
\int_{\Omega}\left|\left(f\left(u_{m_{k}}\right)-f(u)\right) \phi\right| \leq\left\|f^{(3)}\right\|_{\infty} C(\Omega) K^{2}\left\|u_{m_{k}}-u\right\|_{4}
$$

for $\left\|u_{m_{k}}-u\right\|_{4} \leq 1$ and $\phi \in H$ with $\|\phi\|_{H}=1$. Since

$$
\left\|N\left(u_{m_{k}}\right)-N\left(u_{m_{\ell}}\right)\right\|_{H}=\sup _{\phi}\left\langle N\left(u_{m_{k}}\right)-N\left(u_{m_{f}}\right), \phi\right\rangle_{H}
$$

with the sup over all $\phi \in H$ with $\|\phi\|_{H}=1$, the sequence $\left\{N\left(u_{m_{k}}\right)\right\}$ is Cauchy and thus $N$ is compact.

Now we modify the proof of $[\mathrm{II},(2.8)]$ to show that $A$ is proper. Since $(\eta / 3 !)|s|^{3} \leq(\operatorname{sign} s) f(s)(1.10)($ iii $)$,

$$
\int_{\Omega}\left((\eta / 3 !) u_{n}{ }^{4}-\lambda(n) u_{n}{ }^{2}\right) \leq \int_{\Omega}\left(f\left(u_{n}\right) u_{n}-\lambda(n) u_{n}{ }^{2}\right)
$$

We may rewrite $[\mathrm{II},(2.8)(3)]$ as

$$
\left\|g_{n}\right\|_{H}\left\|u_{n}\right\|_{H}^{2} \geq\left\|u_{n}\right\|_{H}^{2}+\int_{\Omega}\left((\eta / 3 !) u_{n}{ }^{2}-\lambda(n)\right) u_{n}{ }^{2},
$$

let $\Omega_{+}=\left\{x \in \Omega: \lambda(n)>(\eta / 3 !)\left(u_{n}(x)\right)^{2}\right\}$, and proceed as before.

2.2. Lemma. Let $f: \mathbb{R} \rightarrow \mathbb{R}$ be $C^{2}$ with $f(0)=0$, and let $\Omega$ be a bounded $C^{1}$ domain in $\mathbb{R}^{n}$. Then

(a) $\eta: C_{0}^{2, \alpha}(\bar{\Omega}) \rightarrow C^{\alpha}(\bar{\Omega}), u \rightarrow f(u)$, and

(b) $\zeta: C_{0}^{2, \alpha}(\bar{\Omega}) \times \mathbb{R} \rightarrow C^{\alpha}(\bar{\Omega}), u \rightarrow-\lambda u+f(u)$ are compact maps.

Proof. (a) If the sequence $\left\{u_{k}\right\}$ is bounded in $C^{2, \alpha}(\bar{\Omega})$, then $\left\{f\left(u_{k}\right)\right\}$ is bounded in $C^{1}(\bar{\Omega})$ [GT, p. 10] and thus in $C^{0, \gamma}(\bar{\Omega}),[Z$, p. 233], where $\alpha<$ 
$\gamma<1$. Since the inclusion of $C^{0, \gamma}(\bar{\Omega})$ in $C^{0, \alpha}(\bar{\Omega})$ is compact [Cr, Lemma 5.11, p. 159], the conclusion results.

(b) If $\left\{\left(u_{k}, \lambda_{k}\right)\right\}$ is bounded, then there are subsequences (still use $\left.k\right) \lambda_{k} \rightarrow \bar{\lambda}$ and $\zeta\left(u_{k}, \bar{\lambda}\right)$ converging in $C_{0}^{\alpha}(\bar{\Omega})$. Since $\zeta\left(u_{k}, \lambda_{k}\right)-\zeta\left(u_{k}, \bar{\lambda}\right) \rightarrow 0$, the conclusion results.

2.3. Notation. In (2.4) assume $\Omega \subset \mathbb{R}^{n}, n$ arbitrary, $\Omega$ connected bounded open and $C^{2, \alpha}$. Define $Y_{j}$ as the span of $\phi_{1}, \ldots, \phi_{j}$ (1.8), $H_{j}$ [resp., $H_{j}^{\prime}$ ] as the $L^{2}$-orthogonal complement of $Y_{j}$ in $C_{0}^{2, \alpha}(\bar{\Omega})$ [resp., $C^{\alpha}(\bar{\Omega})$ ] $(j=$ $0,1, \ldots)$. (Thus $H_{0}=C^{2, \alpha}(\bar{\Omega})$ [resp., $\left.H_{0}^{\prime}=C^{\alpha}(\bar{\Omega})\right]$.) Define $P_{j}: C_{0}^{2, \alpha}(\bar{\Omega}) \rightarrow$ $H_{j}, P_{j}^{\prime}: C^{\alpha}(\bar{\Omega}) \rightarrow H_{j}^{\prime}$ to be projections.

Let $f: \mathbb{R} \rightarrow \mathbb{R}$ be $C^{2}, f(0)=0$, and define

$$
A_{\lambda}: C_{0}^{2, \alpha}(\bar{\Omega}) \rightarrow C^{\alpha}(\bar{\Omega}), \quad u \rightarrow-\Delta-\lambda u+f(u),
$$

and for each fixed $\alpha_{1}, \ldots, \alpha_{j} \in \mathbb{R}$ define

$$
\psi_{j, \lambda}: H_{j} \rightarrow H_{j}^{\prime}, \quad v \rightarrow P_{j}^{\prime} A_{\lambda}\left(\alpha_{1} \phi_{1}+\cdots+\alpha_{j} \phi_{j}+v\right)=w .
$$

Define $A: C_{0}^{2, \alpha}(\bar{\Omega}) \times \mathbb{R} \rightarrow C^{\alpha}(\bar{\Omega}) \times \mathbb{R}$ by $A(u, \lambda)=\left(A_{\lambda}(u), \lambda\right)$ and $\psi_{j}$ : $H_{j} \times \mathbb{R} \rightarrow H_{j}^{\prime} \times \mathbb{R}$ by $\psi_{j}(v, \lambda)=\left(\psi_{j, \lambda}(v), \lambda\right)$.

2.4. Lemma. Assume (2.3) only.

(i) The map $\psi_{j}$ is Fredholm of index 0 for all $\lambda \in \mathbb{R}$ and $j=0,1, \ldots$.

(ii) If $f$ is $C^{k} \quad(k \geq 2)$ and $f^{\prime}(s) \geq 0$ for all real $s$, then $\psi_{j}$ is a $C^{k-1}$ local diffeomorphism for $\lambda \leq \lambda_{j+1} \quad(j=0,1, \ldots)$.

(iii) Assuming (1.10), $\psi_{j}$ is proper for $j=0$ and 1.

(iv) Assuming (1.10), $\psi_{j}$ is a $C^{k-1}$ diffeomorphism for $\lambda<\lambda_{j+1}$ and $j=0$ or 1 .

Proof. (i) From [GT, Theorem 6.14, p. 107] $-\Delta: C_{0}^{2, \alpha}(\bar{\Omega}) \rightarrow C^{\alpha}(\bar{\Omega})$ is invertible and $-\Delta\left(H_{j}\right)=H_{j}^{\prime}$, so $(-\Delta) \times$ id $: H_{j} \times \mathbb{R} \rightarrow H_{j}^{\prime} \times \mathbb{R}$ is invertible. The map $\gamma:(u, \lambda) \rightarrow\left(P_{j}^{\prime} \zeta(u, \lambda), 0\right)(2.2)$ is compact; since $\psi_{j}$ is the sum of these two maps, conclusion i) results [B, p. 100, (2.6.3)(c)].

(ii) Assume $\lambda<\lambda_{j+1}$. Use the argument of [II, (2.2)] to the end of its display, where the $H$ inner product is replaced by the $L^{2}$ inner product, to conclude that $D \psi_{j, \lambda}(v)$ is injective for each $v \in H_{j}$. Conclusion (ii) follows from (i) and the Inverse Function Theorem [B, pp. 113-114].

(iii) Write (1) $A_{\lambda}(u)=A_{\lambda}\left(\alpha_{1} \phi_{1}+\cdots+\alpha_{j} \phi_{j}+v\right)=\beta_{1} \phi_{1}+\cdots+\beta_{j} \phi_{j}+w$, and consider for fixed $\alpha_{1}, \ldots, \alpha_{j} \in \mathbb{R}, \quad \psi_{j}(v, \lambda)=\left(\psi_{j, \lambda}(v), \lambda\right)=(w, \lambda)$. Since $\psi_{j}$ is a compact perturbation of a proper mapping, it suffices [B-2, (2.7.2)(i), p. 103] to prove that $\left(w_{m}, \lambda_{m}\right)$ bounded in $C^{\alpha}(\bar{\Omega}) \times \mathbb{R}$ implies $\left(v_{m}, \lambda_{m}\right)$ is bounded in $C_{0}^{2, \alpha}(\bar{\Omega}) \times R$, i.e. (drop the $m$ ) (2) $\|w\|_{\alpha}$ and $\lambda$ bounded implies $\|u\|_{2, \alpha}$ bounded.

First suppose (3) $\beta_{1}^{2}+\cdots+\beta_{j}^{2}$ is bounded (for all $m$ ). From (1),

$$
-\Delta u=\lambda u-f(u)+\beta_{1} \phi_{1}+\cdots+\beta_{j} \phi_{j}+w .
$$

At a maximum $\bar{x}$ of $u,-\Delta u(\bar{x}) \geq 0$, so that

$$
f(u(\bar{x}))-\lambda u(\bar{x}) \leq\left|\beta_{1}\right|\left\|\phi_{1}\right\|_{0, \alpha}+\cdots+\left|\beta_{j}\right|\left\|\phi_{j}\right\|_{0, \alpha}+\|w\|_{0, \alpha} ;
$$


from hypotheses (1.10), ( $\operatorname{sign} s) f(\eta) \geq \eta|s|^{3} / 3$ !. We obtain an upper bound for $u(\bar{x})$ (in terms of the $w$, the $\beta_{j}$, and the $\lambda$ ). Use a minimum of $u$ also to obtain a bound for $u$ in $L^{\infty}(\Omega)$. By [LU, Theorem 15.1, p. 203] applied to the right side of (4), $u$ is bounded in $C^{0, \alpha}(\bar{\Omega})$ and by the Mean Value Theorem $f(u)$ is bounded in $C^{0, \alpha}(\bar{\Omega})$. From [GT, Theorem 6.6, p. 98] we obtain a bound for $u$ in $C_{0}^{2, \alpha}(\bar{\Omega})$, yielding (2) and thus conclusion (iii) in case (3).

It now suffices to prove (iii) (and thus (2)) in case $j=1$ (with $\beta=\beta_{1}$ ) supposing that $\left(3^{\prime}\right) \quad \beta \rightarrow \infty$ (the case $\beta \rightarrow-\infty$ is similar). Let $\tilde{u}=u-$ $\left(\alpha_{1}+1\right) \phi_{1}\left(\alpha_{1}\right.$ is fixed by hypothesis). Then

$$
-\Delta \tilde{u}+f\left(\tilde{u}+\left(\alpha_{1}+1\right) \phi_{1}\right)-\lambda \tilde{u}=\tilde{\beta} \phi_{1}+w,
$$

where $\tilde{\beta}=\beta+\left(\alpha_{1}+1\right)\left(\lambda_{1}-\lambda\right)$ and $\tilde{\beta} \rightarrow \infty$. By considering where $\tilde{u}$ has its minimum we obtain a lower bound for $\tilde{u}$. Let $T=\{x \in \Omega: \tilde{u}(x)<0\} \quad(T$ may be $\varnothing)$. From (5),

$$
\int_{T}\left[|\nabla \tilde{u}|^{2}+f\left(\tilde{u}+\left(\alpha_{1}+1\right) \phi_{1}\right) \tilde{u}-\lambda \tilde{u}^{2}-w \tilde{u}\right]=\tilde{\beta} \int_{T} \tilde{u} \phi_{1} .
$$

Since $u$ is bounded on $T$, the left side of $(6)$ is bounded below by a bound depending on $\alpha_{1}$ and $\|w\|_{2}$, and $\int_{T} \tilde{u} \phi_{1} \geq-K / \tilde{\beta}$, where $K>0$. Since $\phi_{1}>0$ on $\Omega$ [GT, p. $\left.214,(8.38)\right], \int_{\Omega-T} \tilde{u} \phi_{1} \geq 0$, so that $\int_{\Omega} \tilde{u} \phi_{1} \geq-K / \tilde{\beta}$. Thus $\alpha_{1}=\int_{\Omega} u \phi_{1} \geq\left(\alpha_{1}+1\right)-K / \tilde{\beta}$. Since $\tilde{\beta} \rightarrow \infty$, a contradiction results. Thus $\beta$ is bounded, so the rest of conclusion (ii) results. (In case $\beta \rightarrow-\infty$, use $\tilde{u}=u-\left(\alpha_{1}-1\right) \phi_{1}$, maximum in lieu of minimum, $T=\{x: \tilde{u}(x)>0\}$, but still a lower bound for the left side of (6) so that $\int_{T} \tilde{u} \phi_{1} \leq-K / \tilde{\beta}$.)

(iv) From (ii) and (iii) $\psi_{j}$ for $\lambda<\lambda_{j+1}$ is a finite-to-one covering map [P, p. 128], and since $H_{j}^{\prime}$ is simply connected, conclusion (iv) follows immediately from [M, Theorem 6.6, p. 159 or Exercise 6.1, p. 160].

2.5. Corollary. Assume $f$ is $C^{2}, f(0)=0$, and $\Omega$ is $C^{2, \alpha}$ (Hölder case).

(i) The maps $A$ and $A_{\lambda}$ are Fredholm of index 0 for all $\lambda \in \mathbb{R}$, and $\operatorname{dim} \operatorname{ker} D A_{\lambda}(u)=\operatorname{dim} \operatorname{ker} D A(u, \lambda) \leq j$ for $\lambda<\lambda_{j+1}$.

(ii) Assuming (1.10), $A$ and $A_{\lambda}$ are proper (for all $\lambda \in \mathbb{R}$ ).

(iii) Assuming (1.10), $A$ and $A_{\lambda}$ are $C^{k-1}$ diffeomorphisms for $\lambda<\lambda_{1}$.

\section{ThE MAP $A_{\lambda}$}

We assume hypotheses (1.10), except that in (3.4) the lesser hypotheses given there will suffice. Information relating the maps $A$ and $A_{\lambda}$ is given in (3.1) and (3.2), and (3.9) and (3.13) show that $A_{\lambda}$ satisfies condition (v) of the abstract characterization of the global cusp map $((8.1)$ or $(8.2))$. This information is used to derive $(1.1)$ (b) in (8.5).

3.1. Lemma. Assume that $0 \neq e$ generates $\operatorname{ker} D A_{\lambda}(u)$ and $J(u)=\int_{\Omega} f^{\prime \prime}(u) e^{3}$. Then

(a) $A_{\lambda}$ at $u$ is a fold ((1.5) or [II, (3.1)]) if and only if $J(u) \neq 0$;

(b) $A_{\lambda}$ at $u$ is a cusp ((1.5) or [II, (3.1)]) if and only if $u \neq 0, J(u)=0$ and $D J(u) \cdot e \neq 0$. 
Proof. See Appendix (A.2), (A.3). From [II, (3.4)] (1) the following statements are equivalent: (i) $A_{\lambda}$ at $u$ is a fold, (ii) $A$ at $(u, \lambda)$ is a fold, (iii) some $e \neq 0$ generates $\operatorname{ker} D A_{\lambda}(u)$ and $J(u) \neq 0$. Conclusion (a) results.

From [II, (3.5)] and the first two paragraphs of the proof of [CT-1, (1.8)], (2) the following statements are equivalent: (i) $A_{\lambda}$ at $u \neq 0$ is a precusp [II, (3.1)], (ii) $A$ at $(u, \lambda)$ with $u \neq 0$ is a precusp, (iii) some $e(u)=e \neq 0$ generates $\operatorname{ker} D A_{\lambda}(u), u \neq 0$ and $J(u)=0$. If $y$ is the element defined in the proof of [II, (3.6)], then $D e(u) \cdot e=-y$ by [III, (1.10)(b)]. It follows that

$$
D J(u) \cdot e=\int_{\Omega} f^{(3)}(u) e^{4}-3 \int_{\Omega} f^{\prime \prime}(u) e^{2} y,
$$

which is the displayed expression in the proof of [II, (3.6)]; condition ( $\tilde{4})$ for (intrinsic) cusp [II, (3.1)] is that it is nonzero. Thus (4) the following statements are equivalent:

(i) $A_{\lambda}$ at $u \neq 0$ is a cusp, (ii) $A$ at $(u, \lambda)$ with $u \neq 0$ is a cusp; (iii) $u \neq 0, e \neq 0$ generates $\operatorname{ker} D A_{\lambda}(u), J(u)=0$, and $D J(u) \cdot e \neq 0$. Conclusion (b) results.

3.2. Remark. . If $\lambda_{j}$ is a simple eigenvalue for $-\Delta$ (e.g. $\left.\lambda_{1}\right)$, then

(a) $A_{\lambda_{j}}$ is a local homeomorphism at 0 .

(b) Thus (i) $A_{\lambda_{j}}$ at 0 is not a cusp, while (ii) $A$ at $\left(0, \lambda_{j}\right)$ is a cusp.

As a result the restriction $u \neq 0$ is needed in $(3.1)(b),(2)$ and (4).

Proof. Conclusion (b)(ii) follows from $f^{\prime \prime}(0)=0$ and [II, (3.6)]. Since $S A=$ $\bigcup_{i}$ graph $\lambda_{i}$ [III, (1.5)(a)] and (1) $\lambda_{i}=\lambda_{i}(0)<\lambda_{i}(u)$ for all $u \neq 0(i=$ $1,2, \ldots)$ [III, (2.3)], it follows from the simplicity hypothesis that for some neighborhood $U$ of $\left(0, \lambda_{j}\right)$ in $H \times \mathbb{R}, U \cap$ graph $\lambda_{j}=U \cap S A$. From [II, (3.1)] we may suppose that 2) $U \stackrel{A}{\rightarrow} A(U)$ is $C^{\infty}$ equivalent to the standard cusp map $w \times$ id and so (3) is a proper map. Let $W$ be an open ball in $H$ with center 0 such that $\bar{W} \times \lambda_{j} \subset A(U)$, and let $K$ be the component of $A_{\lambda_{j}}{ }^{-1}(W)$ containing 0 .

By (2) $K \times \lambda_{j} \subset U$, so $K \cap S A_{\lambda_{1}}=\{0\}$ by (1). Since $W-\{0\}$ is simply connected and (by (3)) $A_{\lambda_{j}} \mid K: K \rightarrow W$ is a proper map, the first two paragraphs of the proof of [II, (2.7)(ii)] yield conclusion (a), and thus conclusion (b)(i).

3.3. Remark. The eigenvector $\phi_{i}$ of $-\Delta$ is in $\operatorname{ker} D A_{\lambda}(u)$ if and only if $\lambda=\lambda_{i}$ and $u=0$.

Proof. For every $\psi \in H$,

$$
\begin{aligned}
0 & =\left\langle D A_{\lambda}(u) \cdot \phi_{i}, \psi\right\rangle_{H} \\
& =\int_{\Omega}\left[\nabla \phi_{i} \nabla \psi-\lambda \phi_{i} \psi+f^{\prime}(u) \phi_{i} \psi\right] \\
& =\int_{\Omega}\left[\lambda_{i}-\lambda+f^{\prime}(u)\right] \phi_{i} \psi .
\end{aligned}
$$

Since $\phi_{i} \in L^{\infty}(\Omega)$ [GT, Theorem 8.15, p. 189] and $f^{\prime}(u) \in L^{2}(\Omega)$ (by (1.10) and [A, pp. 97-98]), $\left[\lambda_{i}-\lambda+f^{\prime}(u)\right] \phi_{i} \in L^{2}(\Omega)$; since the above integral is 0 for all $\psi \in C_{0}^{\infty}(\Omega)$ and the latter space is dense in $L^{2}(\Omega)$ [A, Theorem 2.19, p. 31], 
$\left[\lambda_{i}-\lambda+f^{\prime}(u)\right] \phi_{i}=0$ a.e. Since $\phi_{i}$ is real analytic [BJS, p. 136 and pp. 207210], $\phi_{i} \neq 0$ a.e., $\lambda_{i}-\lambda+f^{\prime}(u)=0$ a.e., and so $f^{\prime}(u)$ is constant. Now $f^{\prime}(u)=0$ only where $u=0$ and $f^{\prime}$ is a diffeomorphism on each of $(-\infty, 0)$ and $(0, \infty)$ by $(1.10)$. If the constant $f^{\prime}(u) \neq 0$, then $u$ a.e. takes only two values, one positive and one negative. Since $u \in W_{0}^{1,2}$, this is impossible (cf. the subsequent Lemma (4.4)). Thus $f^{\prime}(u)=0$, so that $u=0$ and $\lambda=\lambda_{i}$.

3.4. Lemma. For $f(u)$ satisfying (1.10)(i) with $f^{\prime}(s) \geq 0$ for all $s \in \mathbb{R}$, and $(u, \lambda)$ having $\operatorname{dim} \operatorname{ker} D A_{\lambda}(u)=1$ with generator $e$ and $\|e\|_{2}=1$,

$$
\int_{\Omega} f^{\prime \prime}(u) e^{2} \phi=D \lambda(u) \cdot \phi
$$

where $\phi \in H$ and $\lambda(u)$ is as given in [III, (1.8)].

Proof. From the proof of [III, (1.8)]

$$
D_{(\lambda, w)} F(\bar{u}, \bar{\lambda}, 0) \cdot(0, \mu, \psi)=-\mu L \bar{v}+D A_{\bar{\lambda}}(\bar{u}) \cdot \psi
$$

(where $\langle L(\phi), \zeta\rangle_{H}=\int_{\Omega} \phi \zeta$ and $\bar{v}=e(\bar{u}, \bar{\lambda})$ ), and from the first two sentences of the proof of [III, (1.10)], for every $\phi \in H$,

$$
\begin{aligned}
-D^{2} A_{\lambda(\bar{u})}(\bar{u})(\bar{v}, \phi) & =D_{(\lambda, w)} F(\bar{u}, \lambda(\bar{u}), 0)[D(\lambda, w)(\bar{u}) \cdot \phi] \\
& =-(D \lambda(\bar{u}) \cdot \phi) L \bar{v}+D A_{\lambda(\bar{u})}(\bar{u}) D w(\bar{u}) \cdot \phi .
\end{aligned}
$$

Take $H$ inner product with $\bar{v}$ to obtain the conclusion.

3.5. Corollary. For any $u, \phi \in H$ and $e=e\left(u, \lambda_{1}(u)\right)$ with $\|e\|_{2}=1$,

$$
\int_{\Omega} f^{\prime \prime}(u) e^{2} \phi=D \lambda_{1}(u) \cdot \phi .
$$

3.6. Lemma. For $0 \neq u \in H$ and $c \geq 0, \lambda_{1}(c u)$ is a strictly increasing function of $c$.

Proof. Since $s f^{\prime \prime}(s)>0$ for $s \neq 0$ (1.10), by (3.5) and the positivity of $e$ [III, (1.6)] $D \lambda_{1}(u) \cdot u>0$ for $u \neq 0$, and the conclusion results. In case $f(u)=u^{3}$, (3.6) is a special case of [III, (2.3)], and yields [III, (2.4)] and [III, (2.5)(b)].

3.7. Lemma. Assume $\left\|f^{(3)}\right\|_{\infty}<\infty$, let $T \subset H \cap L^{\infty}(\Omega)$ be the set of real analytic functions and let $0 \neq u \in T$. Then $\lambda_{1}(c u) \rightarrow \infty$ as $c \rightarrow \infty$.

Proof. Modify the proof of [III, (2.8), p. 209], replacing [III, (2.3)] by (3.6) and noting from (1.10) (iii) that

$$
\left(c^{2} \eta / 2\right) \int_{\Omega} u^{2}(e(c u))^{2} \leq \int_{\Omega} f^{\prime}(c u)(e(c u))^{2} \leq M .
$$

3.8. Lemma. Sobolev case.

(a) Let $T \subset H \cap L^{\infty}(\Omega)$ be the subset of functions real analytic on $\Omega$; then $T$ is dense in $H$.

(b) For $f(u)=u^{3}$ there is a meager (i.e. first category) uncountable subset $X \subset H$ such that for every $u \in H-X, \quad \lambda_{1}(c u) \rightarrow \infty$ as $c \rightarrow \infty$.

Proof. (a) Let $\psi \in H$ and let $\phi_{k} \in H \quad(k=1,2, \ldots)$ be the eigenvectors of $-\Delta$. Then $\phi_{k}$ is real analytic on $\Omega$ [BJS, p. 136 and pp. 207-210], in $W_{0}^{1,2}(\Omega)$ 
for $n \leq 4$ [GT, Theorem 8.3, p. 181], and in $L^{\infty}(\Omega)$ [GT, Theorem 8.15, p. 189]. From [So, Theorem 2, p. 132 and Theorem 5, p. 136], if $\zeta \in H$ and $\left\langle\phi_{k}, \zeta\right\rangle_{H}=0 \quad(k=1,2, \ldots)$, then $\zeta=0$; thus [H, Theorem 1, p. 27]

$$
\sum_{k=1}^{m}\left\langle\phi_{k}, \psi\right\rangle_{H} \phi_{k} \underset{H}{\rightarrow} \psi \text {. }
$$

(b) Define $X \subset H$ by: $u \in H-X$ if and only if $\lambda_{1}(c u) \rightarrow \infty$ as $c \rightarrow \infty$. In the proof of [III, (2.6)] there are an uncountable number of possible choices of the ball $U$, so that $X$ is uncountable. From (a) and the last paragraph of [III, (2.8)], $H-X$ is dense in $H$. Let $Y_{k} \quad(k=1,2, \ldots)$ be the set of $v \in H$ such that for some $c>0, \lambda_{1}(c v)>\lambda_{1}+k$; from [III, (2.9)] $Y_{k}$ is open, and thus is a dense open subset of $H$. Since $H-X=\cap_{k} Y_{k}$, the conclusion results.

3.9. Proposition. Sobolev case. For $\lambda_{1}<\lambda<\lambda_{2},\left(H, S A_{\lambda}\right)$ is homeomorphic ( $C^{\infty}$ diffeomorphic if $f$ is $C^{\infty}$ ) to $\left(H, H_{1}\right)$, where $H_{1}$ is a codimension one subspace of $H$. In fact, for any $\lambda>\lambda_{1}$ this is true for $\left(H, S A_{\lambda} \cap\right.$ graph $\left.\lambda_{1}\right)$.

Proof. Let $S=S A_{\lambda} \cap$ graph $\lambda_{1}$. We will prove that (1) the $j$ th homotopy group $\pi_{j}\left(S, u_{0}\right)=0$ for any $u_{0} \in S$ and $j=0,1, \ldots$. From 1) and [E, p. 766] $S$ is contractible and for $f C^{\infty}$ it is thus [K-2, Theorem 7.3, p. 106] $C^{\infty}$ diffeomorphic to $H_{1}$, and the conclusion results from [K-1, Corollary 6 , p. 89] or [BH, Theorem 0.1, p. 1261]. For $f C^{k}$ one obtains a homeomorphism by [BH, Theorem 0.1, p. 1261].

Since $S=\left\{u \in H: \lambda_{1}(u)=\lambda\right\} \quad([I I I,(1.5)(\mathrm{a})]$ and (3.6)) and 0 is the only critical point of $\lambda_{1}: H \rightarrow \mathbb{R}$ [III, $\left.(1.10)(\mathrm{d})\right], S$ is a $C^{k-1}$ (real analytic if $f(u)=u^{3}$ ) submanifold of $H$ of codimension 1 , and $0 \notin S$ (3.6). For each $0 \neq u \in H$, the ray $L_{u}=\{c u: c>0\}$ meets $S$ in at most one point ([III, (2.5)] and (3.6)) $c(u) u$ and meets it transversely [III, (2.9)]. Let $S^{\prime} \subset H-\{0\}$ be the open set $\{c u: c>0$ and $u \in S\}$, and define $\psi: S \times(0, \infty) \rightarrow S^{\prime}$ by $\psi(u, c)=c u$. Then $\psi$ is a $C^{k-1}$ (real analytic if $f(u)=u^{3}$ ) diffeomorphism, and by (1) it suffices to prove $\pi_{j}\left(S^{\prime}, u_{0}\right)=0$ for any $u_{0} \in S^{\prime}(j=0,1, \ldots)$.

For each $u \in T-\{0\} \quad(3.8), \lambda_{1}(c u) \rightarrow \infty$ as $c \rightarrow \infty$ by (3.7), so there is a $c=c(u)$ with $\lambda_{1}(c u)=\lambda$. Thus

(2) $T-\{0\} \subset S^{\prime}$ and is dense (3.4), so we may suppose $u_{0} \in T \cap S^{\prime}$. Let $\Sigma^{j}$ be the unit sphere in $\mathbb{R}^{j+1}$, let $s_{0}=(1,0, \ldots, 0)$, and let $\zeta:\left(\Sigma^{j}, s_{0}\right) \rightarrow$ $\left(S^{\prime}, u_{0}\right)$ be any map (continuous function). It suffices to show that

(3) there is a homotopy $H_{t}:\left(\Sigma^{j}, s_{0}\right) \rightarrow\left(S^{\prime}, u_{0}\right)$ with $H_{0}=\zeta$ and $H_{1} \equiv u_{0}$.

(4) For each $u \in S^{\prime}$ there is a convex open set $W_{u} \subset S^{\prime}$ such that $u \in W_{u}$. The set of $\zeta^{-1}\left(W_{u}\right)$ for $u \in S^{\prime}$ is an open cover of $\Sigma^{j}$, and there is [W, Theorem 22.5, p. 163] a $\delta>0$ such that

(5) for every $s \in \Sigma^{j}, S(s, \delta) \subset \zeta^{-1}\left(W_{u}\right)$ for some $u \in S^{\prime}$; we may suppose that $\delta$ is small enough that $S(s, \delta)$ is homeomorphic to $\mathbb{R}^{j}$ for every $s \in \Sigma^{j}$.

Triangulate $\Sigma^{j}$ with mesh less than $\delta$, and let $s_{i}(i=0,1, \ldots, r)$ be the vertices (where $s_{0}$ is the base point of $\Sigma^{j}$ ). Thus $\Sigma^{j}$ is homeomorphic to a finite polyhedron $K$ in some Euclidean space, and each point $s \in K$ can be uniquely written as $s=\sum_{i} a_{i} s_{i}$ where $0 \leq a_{i} \leq 1$ and $\sum_{i} a_{i}=1(i=0,1, \ldots, r)$.

(6) If $\sigma$ is the open $m$-simplex whose vertices are $s_{i(0)}, \ldots, s_{i(m)}$, then $s \in \bar{\sigma}$ if and only if $a_{i}(s)=0$ for $i \neq i(0), \ldots, i(m)$ and $s \in \sigma$ if and only 
if $a_{i}(s) \neq 0$ precisely for $i=i(0), \ldots, i(m)$. We will not distinguish between $\Sigma^{j}$ and $K$, i.e. we put these coordinates on $\Sigma^{j}$. Let $W_{i}$ be the $W_{u}$ given by (5) for $s=s_{i}(i=0,1, \ldots, r)$; then

(7) for each simplex $\sigma$ of $\Sigma^{j}$ with vertices $s_{i(0)}, \ldots, s_{i(m)}, \zeta(\bar{\sigma}) \subset W=$ $W_{i(0)} \cap \cdots \cap W_{i(m)}$.

(8) There are (by (3.4)) $u_{i} \in T(i=1, \ldots, r)$ such that (a) $\zeta\left(s_{i}\right) \in W_{j}$ implies $u_{i} \in W_{j}(i, j=0,1, \ldots, r)$ and (b) $\left\{u_{0}, u_{1}, \ldots, u_{r}\right\}$ is linearly independent. Define a homotopy $F_{t}:\left(\Sigma^{j}, s_{0}\right) \rightarrow\left(S^{\prime}, s_{0}\right)$ by

$$
F_{t}\left(\sum_{i} a_{i} s_{i}\right)=(1-t) \zeta\left(\sum_{i} a_{i} s_{i}\right)+t \sum_{i} a_{i} u_{i}
$$

For each $m$-simplex $\sigma$ of $\Sigma^{j}$ with vertices $s_{i(0)}, \ldots, s_{i(m)}$, by (4), (7) and (8)(a) $\zeta(\bar{\sigma})$ and $u_{i(0)}, \ldots, u_{i(m)}$ are contained in $W$, so that $(9) F_{t}(\bar{\sigma}) \subset W$ (and $0 \notin W$ by (4)). From (4), (7) and (9) it follows that (10) $F_{t}(s) \in S^{\prime}$ for every $s \in \Sigma^{j}$.

Let $X$ be the convex hull of $\left\{u_{0}, u_{1}, \ldots, u_{r}\right\}$, so that $X \subset T-\{0\} \subset S^{\prime}$ by (8)(b) and (2) and $F_{1}(s) \in X$ for every $s \in \Sigma^{j}$; define a homotopy $G_{t}$ : $\left(X, u_{0}\right) \rightarrow\left(X, u_{0}\right) \subset\left(S^{\prime}, u_{0}\right)$ by

$$
G_{t}\left(\sum_{i} a_{i} u_{i}\right)=(1-t) \sum_{i} a_{i} u_{i}+t u_{0} .
$$

Define $H_{t}:\left(\Sigma^{j}, s_{0}\right) \rightarrow\left(S^{\prime}, u_{0}\right)$ by $H_{t}=F_{t} * G_{t}\left(F_{1}\right)$, i.e. $H_{t}(s)=F_{2 t}(s)$ for $0 \leq t \leq 1 / 2$ and $H_{t}(s)=G_{2 t-1}\left(F_{1}(s)\right)$ for $1 / 2 \leq t \leq 1$. Condition (3) results.

3.10. Definitions. (a) Let $T \subset C_{0}^{2, \alpha}(\bar{\Omega})$ be the subset of functions real analytic on $\Omega$.

(b) Let $C_{0}(\bar{\Omega})$ be the set of continuous functions $f: \bar{\Omega} \rightarrow \mathbb{R}$ which vanish on $\partial \Omega$; give $C_{0}(\bar{\Omega})$ the sup norm.

3.11. Lemma. The set $T(3.10)$ is dense in $C_{0}(\bar{\Omega})$.

Proof. Let $f \in C_{0}(\bar{\Omega})$. Write $\Omega=\bigcup_{i=1}^{\infty} \Omega_{i}$ where $\Omega_{i}$ is open and $\bar{\Omega}_{i} \subset$ $\Omega_{i+1}$, and use a partition of unity argument to define $r_{i}: \bar{\Omega} \rightarrow[0,1]$ which is continuous, is 1 on $\bar{\Omega}_{i}$ and has $\operatorname{supp}\left(r_{i}\right) \subset \Omega$. Then $r_{i} f \rightarrow f$ uniformly. Use mollifiers of $r_{i} f$ [A, Lemma 2.18(e), pp. 29-30] to find $g_{i} \in C_{0}^{\infty}(\Omega)$ with $g_{i} \rightarrow f$ uniformly.

Thus it suffices to prove: if $h \in C_{0}^{\infty}(\Omega)$ and $h_{m}=\sum_{i=1}^{m}\left(h, \phi_{i}\right)_{2} \phi_{i}$, then $h_{m} \rightarrow h$ uniformly. From integration by parts $(\Delta h)_{m}=\Delta\left(h_{m}\right)$, and $\Delta^{k}\left(h_{m}\right)=$ $\left(\Delta^{k} h\right)_{m} \rightarrow \Delta^{k} h$ in $L^{2}(\Omega)$ by [So, Theorem 5, p. 136] and [H, Theorem 1, p. 27]. Multiply by $\Delta^{-1}$ [GT, Theorem 8.3, p. 181 and Corollary 8.7, p. 183] to obtain $\Delta^{k-1}\left(h_{m}\right) \rightarrow \Delta^{k-1} h$ in $W^{2,2}(\Omega)$ [GT, Theorem 8.8, p. 183] and thus in some $L^{p_{1}}(\Omega)$ [A, p. 97]. If we iterate this procedure and $k$ is sufficiently large, we obtain $h_{m} \rightarrow h$ in $L^{\infty}(\Omega)$ [T, Theorem 4.1, p. 284].

3.12. Lemma. If $K \subset C_{0}^{2, \alpha}(\bar{\Omega})$ is compact and $\varepsilon>0$, then there is a continuous function $P_{\varepsilon}: K \rightarrow C_{0}^{2, \alpha}(\bar{\Omega})$ with $P_{\varepsilon}(K) \subset T$ (3.10) and $\left\|P_{\varepsilon}(x)-x\right\|_{\infty} \leq \varepsilon$ for every $x \in K$.

Proof. The inclusion $j: C_{0}^{2, \alpha}(\bar{\Omega}) \rightarrow C_{0}(\bar{\Omega})$ is continuous, so $K$ is compact in $C_{0}(\bar{\Omega})$. There exist $x_{i} \in C_{0}^{2, \alpha}(\bar{\Omega})(i=1,2, \ldots, k)$ such that, for each $x \in$ 
$K,\left\|x-x_{i}\right\|_{\infty}<\varepsilon$ for some $i$, and by (3.11) we may suppose that $x_{i} \in T$. Let $\bar{P}_{\varepsilon}$ be the Schauder projection $\left[\mathrm{KZ}, \S 18.4\right.$, p. 89] for $j(K) \subset C_{0}(\bar{\Omega}): \bar{P}_{\varepsilon}(x)=$ $\sum_{i=1}^{k} f_{i}(x) x_{i}$, where $f_{i}(x)=\mu_{i}(x) / \sum_{j=1}^{k} \mu_{j}(x)$ with $\mu_{i}(x)=\varepsilon-\left\|x-x_{i}\right\|_{\infty}$ if $\left\|x-x_{i}\right\|_{\infty} \leq \varepsilon$ and $=0$ otherwise. Thus $f_{i} j: K \rightarrow \mathbb{R}$ is continuous and $P_{\varepsilon}=\bar{P}_{\varepsilon} j$ is the desired function.

3.13. Proposition. For the Hölder case (3.9) is true with homeomorphism only. Proof. By the first two paragraphs of the proof of (3.9), it suffices to prove that $\pi_{j}\left(S^{\prime}, u_{0}\right)=0$ for any $u_{0} \in S^{\prime}(j=0,1, \ldots)$. Thus it suffices to prove [Sp, Lemma 1, p. 365]: (1) if $\zeta:\left(\Sigma^{j}, s_{0}\right) \rightarrow\left(S^{\prime}, u_{0}\right)$ is continuous and $B^{j+1}$ is the ball bounded by $\Sigma^{j}$ in $\mathbb{R}^{j+1}$, then there is a continuous extension $\eta: B^{j+1} \rightarrow$ $S^{\prime}$.

From the definition of $\lambda_{1}(u)$ as an infimum [III, (1.1)], for each $\bar{u} \in S^{\prime}$ there is an $\varepsilon(\bar{u})>0$ such that, if $u \in C_{0}^{2, \alpha}(\bar{\Omega})$ and $\|u-\bar{u}\|_{\infty}<\varepsilon$, then $u \in S^{\prime}$; one $\varepsilon>0$ will suffice for all the points of $\zeta\left(\Sigma^{j}\right)$. Let $P_{\varepsilon}$ be as given by $(3.12)$ for $K=\zeta\left(\Sigma^{j}\right)$; then $(1-t) \zeta(s)+t P_{\varepsilon}(\zeta(s))$ is a homotopy between $\zeta$ and $P_{\varepsilon} \zeta: \Sigma^{j} \rightarrow T-0 \subset S^{\prime}$. Since $T-0$ is homeomorphic to $T$ [Kl] the conclusion results.

\section{THE PRECUSP SET IS A MANIFOLD OF CODIMENSION 1 IN THE SINGULAR SET}

As usual, we assume hypotheses (1.10) except where lesser hypotheses are indicated. In this section we prove (1.6), which comes from (4.1), (4.3), (4.6) and (4.8). Here $C A$ stands for the set of precusp points (4.2). Every cusp point is a precusp point, and in other sections where the notation $C A$ is used $(\S \S 6$ and 8 ), there are sufficient hypotheses that all precusp points are cusp points. Thus $C A$ there is the cusp set.

4.1. Remark. The first eigenvalue function $\lambda_{1}: H \rightarrow \mathbb{R}$ is $C^{k}$ [III, (1.9)], and thus its graph is a $C^{k-1}$ submanifold of codimension 1 of $H \times \mathbb{R} \quad(H=$ $W_{0}^{1,2}(\Omega)$, resp. $\left.C_{0}^{2, \alpha}(\bar{\Omega})\right)$. The graph of $\lambda_{1}$ is a component of $S A$ [III, (1.5)(a) and (1.6)] and, since $\lambda_{j}=\lambda_{j}(0) \leq \lambda_{j}(u)$ for all $u \in H$ by the definition [III, (1.1)] of $\lambda_{j}(u) \quad(j=1,2, \ldots),\left\{(u, \lambda) \in S A: \lambda<\lambda_{2}\right\} \subset$ graph $\lambda_{1}$. The set $\left\{u \in H: \lambda=\lambda_{1}(u)\right\}$ is a $C^{k-1}$ manifold of codimension 1 in $H$ [III, (1.10)(c)]; in particular, for $\lambda_{1}<\lambda<\lambda_{2}, S A_{\lambda}$ is a submanifold of codimension 1 in $H$.

4.2. Definition. The set $C A$ [resp., $C A_{\lambda}$ ] of precusp points of $A$ [resp., $A_{\lambda}$ ] consists of those $(u, \lambda) \in H \times R$ [resp., $u \in H$ ] at which $A$ [resp., $A_{\lambda}$ ] is a precusp, i.e. satisfies [II, $(3.1)(1)-(3)]$. By $(3.1)(2)$, if hypotheses (1.10) are satisfied, then $C A \cap$ graph $\lambda_{1}$ is the set of $\left(u, \lambda_{1}(u)\right) \in H \times \mathbb{R}$ with $0=J(u)=$ $\int_{\Omega} f^{\prime \prime}(u) e^{3}$.

4.3. Theorem. (a) For $\lambda<\lambda_{2}$ [resp., $\left.\lambda_{1}<\lambda<\lambda_{2}\right] C A$ [resp., C $\left.A_{\lambda}\right]$ is a $C^{k-2}$ codimension 1 submanifold of $S A$ [resp., $S A_{\lambda}$ ].

(b) More generally, $(C A) \cap\left(\right.$ graph $\left.\lambda_{1}\right)$ is a $C^{k-2}$ codimension 1 submanifold of graph $\lambda_{1}$, and for $\lambda_{1}<\lambda, \quad\left\{u \in C A_{\lambda}: \lambda_{1}(u)=\lambda\right\}$ is a $C^{k-2}$ codimension 1 submanifold of $\left\{u \in H: \lambda_{1}(u)=\lambda\right\}$.

Hypotheses (1.10)(i) and (ii) (without (iii)) suffice. 
Proof. The function $\lambda_{1}: H \rightarrow \mathbb{R}$ is $C^{k-1}$ [III, (1.9)]; we will write $\lambda_{1}(u)$ as $\lambda(u)$ and define $\bar{\lambda}: H \rightarrow H \times \mathbb{R}$ by $\bar{\lambda}(u)=(u, \lambda(u))$. Now $\bar{\lambda}$ maps $H$ diffeomorphically onto graph $\lambda$, which is a component of $S A$ [III, (1.5)(a) and (1.6)], so for every $u \in H$

$$
D \bar{\lambda}(u): H \approx T_{(u, \lambda(u))} S A, \quad z \rightarrow(z, D \lambda(u) \cdot z) .
$$

Using $\bar{\lambda}$ we can view $C A$ as a subset of $H$. Let $u \in(C A) \cap(\operatorname{graph} \lambda)$, and let $e=e(u)>0$ (see [III, (1.8)]) generate $\operatorname{ker} D A_{\lambda(u)}(u)$.

Define $J: H \rightarrow \mathbb{R}$ by $J(u)=\int_{\Omega} f^{\prime \prime}(u) e^{3}$, so $C A$ is the set of zeros of $J$ and $C A_{\lambda}$ (for $\lambda>\lambda_{1}$ ) is the set of zeros of $J \mid\{w: \lambda(w)=\lambda\}$. From [III, (1.8)] $e=e(u)$ is $C^{k-1}$. Let $h: H \rightarrow \mathbb{R}$ be the bounded linear functional given by

$$
h(z)=D J(u) \cdot z=\int_{\Omega} f^{(3)}(u) e^{3} z+3 \int_{\Omega} f^{\prime \prime}(u) e^{2} D e(u) \cdot z .
$$

From the implicit function theorem $[Z$, pp. 150-151] it suffices to prove that

(3) $h(z) \neq 0$ for some $z \in H$ [resp., $z \in T_{u}\{w: \lambda(w)=\lambda(u)\}=T_{u} S A_{\lambda(u)}$ ].

If $u=0$, then $\lambda=\lambda(0)=\lambda_{1}$, and from hypotheses $(1.10), h(e)>0$. Thus we may suppose that $u \neq 0$, so that $\lambda=\lambda(u)>\lambda_{1}$ and it suffices to prove the bracketed statement in (3).

By (1) and [III, (1.10)(d)],

(4) for $u \neq 0, z \in T_{u} S A_{\lambda(u)}$ if and only if $D \lambda(u) \cdot z=0$. From the proof of [III, (1.10)] it follows that

(5) $D \lambda(u) \cdot z=0$ if and only if $D^{2} A_{\lambda(u)}(e, z) \in H_{1}=\{e\}^{\perp_{H}}$, i.e. $\int_{\Omega} f^{\prime \prime}(u) e^{2} z$ $=0$. Define a bounded linear functional $h_{1}: H \rightarrow \mathbb{R}$ by $h_{1}(z)=\int_{\Omega} f^{\prime \prime}(u) e^{2} z$. Thus

(6) the nullspace $N\left(h_{1}\right)$ is the tangent space $T_{u} S A_{\lambda(u)}$.

Define a bounded linear functional $h_{2}: N\left(h_{1}\right) \rightarrow \mathbb{R}$ by

$h_{2}(z)=\int_{\Omega} f^{(3)}(u) e^{3} z-3\left\langle D^{2} A_{\lambda(u)}(u)(e, e),\left(D A_{\lambda(u)}(u)\right)^{-1}\left(D^{2} A_{\lambda(u)}(u)(e, z)\right)\right\rangle_{H}$ since $D A_{\lambda(u)}(u): H_{1} \approx H_{1},\left(D A_{\lambda(u)}(u)\right)^{-1}\left(D^{2} A_{\lambda(u)}(u)(e, z)\right)$ is defined by (4), (5) and (6) up to a multiple of $e, h_{2}$ is well defined on $N\left(h_{1}\right)$ (but not on $H$ ) by (5). Define a bounded linear functional $h_{3}: H \rightarrow \mathbb{R}$ by

$$
h_{3}(z)=\int_{\Omega} f^{(3)}(u) e^{3} z-3\left\langle\left(D A_{\lambda(u)}(u)\right)^{-1}\left(D^{2} A_{\lambda(u)}(u)(e, e)\right), D^{2} A_{\lambda(u)}(u)(e, z)\right\rangle_{H}
$$

(where the inverse is taken in $\left.H_{1}\right)$. By the selfadjointness of $\left(D A_{\lambda(u)}(u)\right)^{-1}$ on $H_{1}$,

(7) $h_{3}(z)=h_{2}(z)$ for every $z \in T_{\mathcal{H}} S A_{\lambda(u)}=N\left(h_{1}\right)$. In the proof of [III, (1.10)] our $e=e(u)$ is (we may suppose) $v(u)=\bar{v}+w(u)$, and thus (by (5)) for all $z \in N\left(h_{1}\right)$,

$$
\left[D A_{\lambda(u)}(u)\right]^{-1}\left(D^{2} A_{\lambda(u)}(u)(e, z)\right)=-D e(u) \cdot z,
$$

and

(9) $h_{2}(z)=h(z)$ (for all $z \in N\left(h_{1}\right)$ ).

From (3), (6), (7) and (9) it suffices to prove that $h_{3}(z) \neq 0$ for some $z \in$ $T_{u} S A_{\lambda(u)}=N\left(h_{1}\right)$. Suppose the contrary; then 
(10) for some $\alpha \in \mathbb{R}, h_{3}(z)=\alpha h_{1}(z)$ for all $z \in H$.

We will obtain a contradiction of $(10)$.

If $y=-D e(u) \cdot e=\left(D A_{\lambda(u)}(u)\right)^{-1}\left(D^{2} A_{\lambda(u)}(u)(e, e)\right)$ [II, proof of (3.6)], then (10) becomes

$$
\int_{\Omega} f^{(3)}(u) e^{3} z-3 \int_{\Omega} f^{\prime \prime}(u) y e z=\alpha \int_{\Omega} f^{\prime \prime}(u) e^{2} z
$$

for all $z \in H$. Since $C_{0}^{\infty}(\Omega)$ is dense in $L^{2}(\Omega)$ [A, p. 31], $H$ is dense in $L^{2}(\Omega)$, and since $e>0$ a.e. on $\Omega$ [III, (1.6)],

$$
f^{(3)}(u) e^{2}=f^{\prime \prime}(u)[3 y+\alpha e]
$$

for almost all $x \in \Omega$.

It follows from [T, Corollary 5.5, p. 303] that both $e$ and $y$ are continuous on $\Omega$. If $\Omega_{1} \subset \subset \Omega$, then [GT, Corollary 8.21 , p. 199 and p. 209, paragraph after Theorem 8.31] $e$ has a positive lower bound on $\Omega_{1}$. It follows from (11) that there exists $K\left(\Omega_{1}\right)>0$ such that

$$
\left|f^{(3)}(u(x))\right| \leq K\left(\Omega_{1}\right)\left|f^{\prime \prime}(u(x))\right|
$$

a.e. on $\Omega_{1} \subset \subset \Omega$.

Since $e>0$ a.e. [III, (1.6)] and by hypothesis $s f^{\prime \prime}(s)>0$ for $s \neq 0$ and $f^{\prime \prime}(0)=0, f^{\prime \prime}(u) e^{3}$ is $>0,<0,=0$ at $x \in \Omega$ if and only if $u$ is $>0$, $<0,=0$, respectively, at $x$ a.e. We are assuming $u \not \equiv 0$ a.e., so that $f^{\prime \prime}(u) e^{3} \not \equiv$ 0 a.e. Since $u \in C A_{\lambda}, \int_{\Omega} f^{\prime \prime}(u) e^{3}=0$, and thus there are subsets $A_{1}$ and $A_{2}$ of positive measure where $u(x)>0$ and $<0$, respectively. There exist compact subsets $B_{1}$ and $B_{2}$ of $\Omega$ of positive measure with the same properties as $A_{1}$ and $A_{2}$, since $m\left(A_{i}\right)=\sup \left\{m\left(B_{i}\right): B_{i} \subset A_{i}, B_{i}\right.$ compact $\}$. Choose $\Omega_{1}$ connected open such that $\bar{\Omega}_{1} \subset \Omega$ and $B_{1} \cup B_{2} \subset \Omega_{1}$. By the following lemma (4.4), for every $\delta>0, X_{\delta}=\left\{x \in \Omega_{1}:|u(x)| \leq \delta\right\}$ has positive measure. By hypothesis $f^{(3)}(s)>0$ for all real $s$, so there exists $\eta>0$ such that $f^{(3)}(u(x)) \geq \eta$ for all $x \in X_{1}$. Choose $0<\varepsilon<\eta / K\left(\Omega_{1}\right)$ (12), and choose $\delta, 0<\delta \leq 1$, such that $|s|<\delta$ implies $\left|f^{\prime \prime}(s)\right|<\varepsilon$. Then (12) fails on $X_{\delta}$, yielding the desired contradiction.

4.4. Lemma. Let $u \in W^{1,1}(\Omega)$, where $\Omega$ is connected and open in $\mathbb{R}^{n}$. If $\{x \in \Omega: u(x)<0\}$ and $\{x \in \Omega: u(x)>0\}$ both have positive measure, then for every $\delta>0,\{x \in \Omega:|u(x)| \leq \delta\}$ has positive measure.

Proof. Suppose the contrary, i.e. there exists $\alpha>0$ such that $\{x \in \Omega:|u(x)| \leq$ $\alpha\}$ has measure zero. Choose $r: \mathbb{R} \rightarrow \mathbb{R} C^{1}$ such that $r$ is odd and $r(t)=1$ if $t \geq \alpha$. From [GT, Theorem 7.8, p. 153] $r(u) \in W^{1,1}(\Omega)$. Since $|u(x)| \geq \alpha$ a.e., $\nabla r(u)=0$ a.e., so that [GT, Lemma 7.16, p. 162] $r(u)$ is locally constant; since $\Omega$ is connected, $r(u)$ is constant, contradicting the properties of $u$ and $r$.

4.5. Remark. For a $C^{k} \quad(k \geq 3)$ map $A: U \rightarrow F$, where $E, F$ are Banach spaces and $U$ is open in $E$, define $C=C A$ to be the set of points satisfying [II, (3.1)(1)-(3)]. In [LM, Theorem 3] the authors prove that if $u \in C$ and a certain linear functional $\psi$ defined on the tangent space

$$
T_{u} S A=\left\{z \in E: D^{2} A(u)(z, e) \in \text { Range } D A(u)\right\}
$$


[LM, Theorem 1] is nonzero, then $C$ is a $C^{k-2}$ submanifold of codimension 2 in a neighborhood of $u$ in $E$.

Consider $A_{\lambda}$ for $f(u)=u^{3}$, let $u \in C=C A_{\lambda}$ and let $z \in T_{u} S A_{\lambda}$. Define $\Gamma_{z} \in H_{1}$ (the $H$-orthogonal complement of (e) by

$$
D A_{\lambda}(u) \cdot \Gamma_{z}=-D^{2} A_{\lambda}(u)(z, e) ;
$$

then from the proof of [III, (1.10)],

(1) $D e(u) \cdot z=\Gamma_{z}($ and $D \lambda(u) \cdot z=0)$. For $z, w \in T_{u} S A_{\lambda}$,

$$
\begin{aligned}
\left\langle D^{2} A_{\lambda}(u)\left(\Gamma_{z}, w\right), e\right\rangle_{H} & =6 \int_{\Omega} u e w \Gamma_{z}=\left\langle D^{2} A_{\lambda}(u)(e, w), \Gamma_{z}\right\rangle_{H} \\
& =-\left\langle D A_{\lambda}(u) \Gamma_{w}, \Gamma_{z}\right\rangle_{H},
\end{aligned}
$$

and the selfadjointness of $D A_{\lambda}(u)$ implies

$$
\left\langle D^{2} A_{\lambda}(u)\left(\Gamma_{z}, w\right), e\right\rangle_{H}=\left\langle D^{2} A_{\lambda}(u)\left(\Gamma_{w}, z\right), e\right\rangle_{H} .
$$

Let the linear functional $\gamma_{0}$ in [LM] be defined by $\left\langle x, \gamma_{0}\right\rangle=\langle x, e\rangle_{H}$ for all $x \in H$; from (2) the functional of [LM, Theorem 3]

$$
\psi(z)=6\left[\int_{\Omega} z e^{3}+3 \int_{\Omega} u e^{2} \Gamma_{z}\right] .
$$

If $J(u)=6 \int_{\Omega} u e^{3}$, it follows from (1) that $\psi(z)=D J(u) \cdot z$, the functional we considered in the proof of (4.3).

Thus, there is a connection between [LM, Theorem 3] and (4.3) but this does not seem to be a feasible way to approach our theorem: we would still have to prove that the functional $\psi$ is nonzero, and that is the essence of our proof.

4.6. Proposition. (Sobolev case). Assume that $f$ satisfies (1.10)(i), (ii) with $k>n+2, n \leq 4$ and $\partial \Omega C^{\infty}$. Then there is an open dense subset $\Gamma \subset S A$ such that every $(u, \lambda) \in \Gamma$ is a fold point of $A$ (and $u$ is a fold point of $A_{\lambda}$ ).

Proof. Let $\Gamma \subset S A$ be the set of fold points; then $\Gamma$ is open in $S A$ and it suffices to prove that it is dense. By [III, (1.12)] there is a dense open subset $\Gamma^{\prime} \subset S A$ such that $\operatorname{dim} \operatorname{ker} D A_{\lambda}(u)=1$ for every $(u, \lambda) \in \Gamma^{\prime}$. At each point $\Gamma^{\prime}$ is locally the graph of a $C^{k-1}$ (real analytic if $f(u)=u^{3}$ ) eigenvalue map $\lambda_{j}$ [III, (1.8)]. Let $\Lambda$ be the set of $u \in C_{0}^{\infty}(\Omega)$ such that $\left(u, \lambda_{j}(u)\right) \in \Gamma^{\prime}$ and (1) $e\left(u, \lambda_{j}(u)\right)$ is a Morse function; by [III, (1.11)-see the first sentence of the proof] the set of $\left(u, \lambda_{j}(u)\right)$ for $u \in \Lambda$ is dense in $\Gamma^{\prime}$.

By [II, (3.5)] each point $(u, \lambda)$ of $\Gamma^{\prime}$ is either a fold point or a precusp point [II, (3.1)] of $A$; in fact, each $u$ with $(u, \lambda) \in \Gamma$ is either a fold point or a precusp point of $A_{\lambda}$, unless $u=0$ and $\lambda=\lambda_{i} \quad(i=1,2, \ldots)$ by (3.1). Let $u \in \Lambda$ be a precusp point. It suffices to prove that (2) the germ (1.3) of the precusp set at $u$ is a codimension one submanifold of $H$. We suppose the contrary and follow the proof of (4.3) up to (11), multiplied by $e$. From (1.10) $f^{\prime \prime}(u(x))$ has compact support in $\Omega$, so from $(4.3)(11) e$ has compact support in $\Omega$, contradicting (1).

4.7. Lemma. Let $V$ be a connected open subset of a topological space $X$ and let $\Lambda \subset X$ be closed with int $\Lambda=\varnothing$. Suppose that for every $x \in V$, there is a 
connected open neighborhood $U(x) \subset V$ of $x$ such that $U(x)-\Lambda$ is connected. Then $V-\Lambda$ is connected.

Proof. Let $W$ be a component of $V-\Lambda$. If $W \neq V-\Lambda$, then $V \cap \bar{W} \neq V$ and there exists $x \in V \cap$ bdy $\bar{W}$; since $W \cap U(x) \neq \varnothing, \quad W \cup(U(x)-\Lambda)$ is connected, and $U(x) \subset \bar{W}$, contradicting the choice of $x$.

4.8. Proposition. Sobolev case for $f$ satisfying (1.10)(i), (ii) with $k \geq n+$ $2, n \leq 4$ and $\partial \Omega C^{\infty}$.

(a) In $H \times \mathbb{R}, \operatorname{int}(S A)=\varnothing$, i.e., $S A$ is meager.

(b) Let $\Lambda \subset S A$ be the set of nonfold points. Then, for every nonempty connected open subset $V$ of $H \times \mathbb{R}, V-\Lambda$ is nonempty connected open, i.e., $\Lambda$ is supermeager in $H \times \mathbb{R}$ [CT-1, (1.5)].

Proof. (a) Since the set of fold points is a dense open subset of $S A$ (4.6), conclusion (a) is immediate.

(b) Let $x \in V$. Since $S A=\bigcup_{j=1}^{\infty}$ graph $\lambda_{j}$ [III, (1.5)] and $\lambda_{j}(u) \geq \lambda_{j}$ for every $u \in H$ from [III, (1.1)], there exist $k>0$ and a connected open neighborhood $U$ of $x$ in $H \times \mathbb{R}$ such that $U \cap\left(\right.$ graph $\left.\lambda_{j}\right)=\varnothing$ for $j>k$. Define $U_{m}=U-\left(\Lambda \cap \bigcup_{j=1}^{m}\right.$ graph $\left.\lambda_{j}\right) \quad(m=0,1, \ldots, k) ; U_{0}=U$. Suppose that $U_{m}$ is connected $(m=0,1, \ldots, k-1)$. By the argument of (4.6) $\Lambda \cap$ graph $\lambda_{j}$ is a closed subset nowhere dense in graph $\lambda_{j}$, and it follows from (4.7) that $U_{m+1}$ is connected. By induction $U-\Lambda=U_{k}$ is connected, and by (4.7) $V-\Lambda$ is connected.

\section{The ONLY Singular points of $A$ ARE fOLdS AND POSITIVE CUSPS}

We assume hypotheses (1.10), except that only parts of it are assumed in (5.1) and (5.4). In (3.1) $J(u)=\int_{\Omega} f^{\prime \prime}(u) e^{3}$ is defined and used to characterize fold point and cusp point of $A_{\lambda}$ and $A$. In this section we prove (5.2) that for $\lambda_{1}(u)$ sufficiently near $\lambda_{1}$, the only singular points of $A$ are folds and positive cusps, i.e. $D J(u) \cdot e>0$. Information relating derivatives of $\lambda_{1}: H \rightarrow \mathbb{R}$ to $J$ is given in (5.3), and (5.4) shows that $e(u) \rightarrow \phi_{1}$, the first eigenfunction of $-\Delta$, in $H$ as $\lambda_{1}(u) \rightarrow \lambda_{1}$. These results are used in the sequel to derive the main theorem (1.1).

5.1. Lemma. Assume hypotheses (1.10)(i) and $f^{\prime}(s) \geq 0$ for all real $s$ (e.g. $\left.f(u)=u^{3}\right)$. If $e \in \operatorname{ker} D A(u, \lambda)$ with $\|e\|_{2}=1$, then $e \in L^{\infty}(\Omega)$ with $\|e\|_{\infty} \leq$ $\lambda^{i} K(\Omega)$, where $K(\Omega)$ is a contant depending only of $\Omega, i=1$ for $n \leq 3$ and $i=3 / 2$ for $n=4$. In the Hölder case, $\|e\|_{\infty} \leq \lambda^{i(n)} K(\Omega)$ for all $n$ and for some $i(n)>0$.

More generally, the lemma holds if $e \in W_{0}^{1,2}(\Omega)$ is a solution of $-\Delta e+a e-$ $\lambda e=0$ on $\Omega$, where $a \in L^{2}(\Omega)$ with $a(x) \geq 0$ for all $x \in \Omega$.

Proof. The map (1) $(-\Delta)^{-1}: W^{-1,2}(\Omega) \rightarrow W_{0}^{1,2}(\Omega)=H$ is a well defined bounded linear isomorphism ([A, (3.8), p. 48 and (3.10) and (3.11), p. 50] and [GT, Theorem 8.3, p. 181 and Corollary 8.7, p. 183]). (2) If $f \in W^{-1,2}(\Omega)$ and $f \geq 0$ [Sc, Chapitre Premier, $\S 4$, p. 28], then $(-\Delta)^{-1} f \geq 0$ by [GT, Theorem 8.1, p. 179]. By [Ka-2, Lemma A, p. 138] $\Delta|e| \geq(\operatorname{sign} e) \Delta e$, where sign $e(x)=0$ if $e(x)=0$. Thus

$$
\Delta|e| \geq-\lambda|e|+f^{\prime}(u)|e| \geq-\lambda|e|,
$$


so that $-\Delta|e| \leq \lambda|e|$ and (by (2)) (3) $|e| \leq \lambda(-\Delta)^{-1}(|e|)$. For $n \leq 3$ it follows from (1), $\|e\|_{2}=1$ and [GT, Theorem 8.15, p. 189] that $\left\|(-\bar{\Delta})^{-1}(|e|)\right\|_{\infty} \leq$ $C(\Omega)$. Since $\|e\|_{H} \leq \lambda^{1 / 2}$ (use $\left\langle D A_{\lambda}(u) \cdot e, e\right\rangle_{H}=0$ ), for $n=4$ it follows from [A, pp. 97-98] and [GT, Theorem 8.15, p. 189] that

$$
\left\|(-\Delta)^{-1}(|e|)\right\|_{\infty} \leq C_{1}(\Omega)+\lambda^{1 / 2} C_{2}(\Omega) \leq \lambda^{1 / 2} C_{3}(\Omega)
$$

(since $\left.0<\lambda_{1} \leq \lambda\right)$. The conclusion results from (3).

For the Hölder case, start with $L^{2}(\Omega)$ and use [T, Theorem 4.1, p. 284] to iterate $L^{q}(\Omega) \rightarrow L^{p}(\Omega)$ until $p$ is sufficiently large to embed in $L^{\infty}(\Omega)$ [GT, (8.15), p. 189]. Then $\left\|(-\Delta)^{i(n)}(|e|)\right\|_{\infty} \leq K(\Omega)$, where $i(n)-1$ is the number of iterations and the conclusion results from (3) and (2).

5.2. Proposition. Assume $\left\|f^{(3)}\right\|_{\infty}<\infty$. There exists $\bar{\lambda}>\lambda_{1}$ such that for every $\lambda \leq \bar{\lambda}$, every singular point $u$ of $A_{\lambda}$ with $\lambda=\lambda_{1}(u)$ is either a fold point, or a cusp point of $A$ with $D J(u) \cdot e>0$, i.e.

$$
\int_{\Omega} f^{(3)}(u) e^{4}-3 \int_{\Omega} f^{(3)}(u) e^{2} y>0
$$

(see [II, proof of (3.6)]).

Proof. From the hypothesis

$$
\left|f^{\prime \prime}(u)\right| \leq\left\|f^{(3)}\right\|_{\infty}(2 / \eta)^{1 / 2}\left(f^{\prime}(u)\right)^{1 / 2} .
$$

Since $\|e\|_{2}=1$ and

$$
\begin{gathered}
{\left[\int_{\Omega}|\nabla e|^{2}-\lambda_{1} \int_{\Omega} e^{2}\right]+\int_{\Omega}\left[f^{\prime}(u)-\left(\lambda_{1}(u)-\lambda_{1}\right)\right] e^{2}=0,} \\
\int_{\Omega} f^{\prime}(u) e^{2} \leq\left(\lambda_{1}(u)-\lambda_{1}\right) .
\end{gathered}
$$

Now $1=\left(\int_{\Omega} e^{2} \cdot 1\right)^{2} \leq m(\Omega) \int_{\Omega} e^{4}$ so that

$$
\int_{\Omega} f^{(3)}(u) e^{4} \geq \eta / m(\Omega) .
$$

Let $\lambda_{i}(u)$ and $v_{i}(u)$ be the eigenvalues and eigenvectors of $-\Delta v-\lambda v+$ $f^{\prime}(u) v=0$ [III, (1.2)], so that $e=v_{1}(u)$ and $\lambda=\lambda_{1}(u)<\lambda_{2}(u) \leq \lambda_{i}(u)$ for every $i>2$ [III, (1.6)]. Let $\tilde{L}$ and $\tilde{H}$ be the $L^{2}(\Omega)$ orthogonal complement of $e$ in $L^{2}(\Omega)$ and $H=W_{0}^{1,2}(\Omega)$, respectively. Define $\Phi: \tilde{L} \rightarrow \tilde{H}$ by

$$
\left\langle D A_{\lambda}(u) \cdot(\Phi g), \psi \psi\right\rangle_{H}=\langle g, \psi\rangle_{2},
$$

so that

$$
\Phi g=\sum_{i=2}^{\infty}\left(\lambda_{i}(u)-\lambda\right)^{-1}\left\langle g, v_{i}(u)\right\rangle_{2} v_{i}(u)
$$

and

$$
\|\Phi g\|_{2}{ }^{2} \leq\left(\lambda_{2}(u)-\lambda\right)^{-2} \sum_{i=2}^{\infty}\left|\left\langle g, v_{i}(u)\right\rangle_{2}\right|^{2}=\left(\lambda_{2}(u)-\lambda\right)^{-2}\|g\|_{2}{ }^{2} .
$$

Let $\left(u, \lambda_{1}(u)\right)$ be a precusp point [II, (3.1)] of $A$; then 
(4) $y=y(u) \in\left(D A_{\lambda}(u)\right)^{-1}\left(D^{2} A_{\lambda}(u)(e, e)\right)$ [II, proof of (3.6)],

and any two such elements differ by a multiple of $e$. We may uniquely specify $y \in \tilde{H}$. By (5.1) $f^{\prime \prime}(u) e^{2} \in L^{2}(\Omega)$, and by (4) $\Phi\left(f^{\prime \prime}(u) e^{2}\right)=y$, so that

$$
\begin{aligned}
\|y\|_{2} & \leq\left(\lambda_{2}(u)-\lambda_{1}(u)\right)^{-1}\left\|f^{\prime \prime}(u) e^{2}\right\|_{2} \\
& \leq\left(\lambda_{2}(u)-\lambda_{1}(u)\right)^{-1}\left(\lambda_{1}(u)-\lambda_{1}\right)^{1 / 2}\left(\lambda_{1}(u)\right)^{i} C,
\end{aligned}
$$

where $C=\left\|f^{(3)}\right\|_{\infty}(2 / \eta)^{1 / 2} K(\Omega)$ by $(5.1),(1)$ and (2). For $\lambda=\lambda_{1}(u) \leq \bar{\lambda}<\lambda_{2}$,

$$
\|y\|_{2} \leq K(f, \Omega)\left(\lambda_{2}-\bar{\lambda}\right)^{-1}\left(\lambda_{1}(u)-\lambda_{1}\right)^{1 / 2}
$$

(since $\lambda_{2} \leq \lambda_{2}(u)$ for all $u \in H$ ), and thus

(5) $\left|\int_{\Omega} f^{\prime \prime}(u) e^{2} y\right| \leq\left\|f^{\prime \prime}(u) e\right\|_{2}\|e\|_{\infty}\|y\|_{2} \leq C(f, \Omega) \bar{\lambda}^{i}\left(\lambda_{2}-\bar{\lambda}\right)^{-1}\left(\lambda_{1}(u)-\lambda_{1}\right)$

by (5.1), (1) and (2). The conclusion results from (3) and (5) (and the fact (4) that $y+c e=-D e(u) \cdot e[\mathrm{III},(1.10)(\mathrm{b})]$ for some $c \in \mathbb{R}$, while $\left.\int_{\Omega} f^{\prime \prime}(u) e^{3}=0\right)$.

5.3. Lemma. (a) For any $u, \phi \in H$ and $e=e\left(u, \lambda_{1}(u)\right)$ with $\|e\|_{2}=1$,

$$
\int_{\Omega} f^{\prime \prime}(u) e^{2} \phi=D \lambda_{1}(u) \cdot \phi .
$$

(b) Thus $J(u)=D \lambda_{1}(u) \cdot e$, where $J(u)=\int_{\Omega} f^{\prime \prime}(u) e^{3}$, and

(c) $D J(u) \cdot e=D^{2} \lambda_{1}(u)(e, e)$. Moreover, if $\left\|f^{(3)}\right\|_{\infty}<\infty$, then

(d) $\left|D \lambda_{1}(u) \cdot \phi\right| \leq\left[C(f, \Omega)\left(\lambda_{1}(u)-\lambda_{1}\right)\right]^{1 / 2}\left(\lambda_{1}(u)\right)^{i}\|\phi\|_{2}$, and

(e) $0 \leq D \lambda_{1}(u) \cdot u \leq\left(2\left\|f^{(3)}\right\|_{\infty} / \eta\right)\left(\lambda_{1}(u)-\lambda_{1}\right)$, where $i$ is given in (5.1).

Proof. Conclusion (a) is (3.5) and conclusions (a), (b) and (c) are immediate from (3.4). For (d) use $\left|\int_{\Omega} f^{\prime \prime}(u) e^{2} \phi\right| \leq\left\|f^{\prime \prime}(u) e\right\|_{2}\|e\|_{\infty}\|\phi\|_{2},(5.1)$, and (1) and (2) in the proof of (5.2). For (e) set $\phi=u$ in (a) and note that the integrand is nonnegative by $(1.10)$ and [III, (1.6)]. Since $\left|f^{\prime \prime}(u) u\right| \leq\left(2\left\|f^{(3)}\right\|_{\infty} / \eta\right) f^{\prime}(u)$, conclusion (e) follows from $(5.2)(2)$.

5.4. Lemma. Assume hypotheses (1.10)(i) with $H=W_{0}^{1,2}(\Omega), f^{\prime}(s) \geq 0$ for all $s \in \mathbb{R}$ and $\lambda \leq \lambda_{2}$.

(i) For $\theta$ the angle between $e$ and $\phi_{1}$ in $H$,

$$
\left[\lambda_{1}\left(\lambda_{2}-\lambda\right) / \lambda\left(\lambda_{2}-\lambda_{1}\right)\right]^{1 / 2} \leq \cos \theta
$$

(ii) $\left\|e-\phi_{1}\right\|_{H} \rightarrow 0$ as $\lambda \rightarrow \lambda_{1}$.

More generally, the lemma holds if $e \in W_{0}^{1,2}(\Omega)$ is a solution of $-\Delta e+a e-$ $\lambda e=0$ on $\Omega$, where $a \in L^{2}(\Omega)$ with $a(x) \geq 0$ for all $x$.

Proof. Since $\int_{\Omega}\left[|\nabla e|^{2}+\left(f^{\prime}(u)-\lambda\right) e^{2}\right]=0$,

$$
\int_{\Omega}|\nabla e|^{2}-\lambda \int_{\Omega} e^{2} \leq 0 .
$$


Write $e=\alpha \phi_{1}+v$, where $\left\|\phi_{1}\right\|_{2}=\|e\|_{2}=1$ and $v \in H_{1}=\left\{\phi_{1}\right\}^{\perp}$, so that $\alpha=\int_{\Omega} e \phi_{1}>0$ by [GT, Theorem 8.38, p. 214] and [III, (1.6)]. By (1) and [GT, (8.94), p. 213]

$$
\left(\lambda_{2}-\lambda\right) \int_{\Omega} v^{2} \leq \int_{\Omega}|\nabla v|^{2}-\lambda \int_{\Omega} v^{2} \leq\left(\lambda-\lambda_{1}\right) \alpha^{2}
$$

and, since $1=\alpha^{2}+\int_{\Omega} v^{2}$,

$$
\left[\left(\lambda_{2}-\lambda\right) /\left(\lambda_{2}-\lambda_{1}\right)\right]^{1 / 2} \leq \int_{\Omega} e \phi_{1} \leq \lambda_{1}^{-1}\left\langle e, \phi_{1}\right\rangle_{H} .
$$

Now $\left\|\phi_{1}\right\|_{H}=\lambda_{1}^{1 / 2}$, (from (1)) $\|e\|_{H} \leq \lambda^{1 / 2}$, and the conclusions result. In fact, from (2) it follows that

$$
\left\|e-\phi_{1}\right\|_{H}^{2} \leq \lambda+\lambda_{1}-2 \lambda_{1}\left[\left(\lambda_{2}-\lambda\right) /\left(\lambda_{2}-\lambda_{1}\right)\right]^{1 / 2} .
$$

\section{THE RESTRICTION MAP $A \mid S A$ IS INJECTIVE}

In a series of lemmas $((6.1)-(6.5))$ we study $u, v \in H$ with $A_{\lambda}(u)=A_{\lambda}(v)$, where $\lambda$ is near $\lambda_{1}$. Later (6.4) we add the hypothesis $u \in S A_{\lambda}$, and finally (6.5) we obtain a contradiction if $v \in S A_{\lambda}$ also. Throughout we assume hypotheses (1.10).

6.1. Lemma. Suppose $a \in L^{2}(\Omega), a(x) \geq 0$ for all $x \in \Omega$, and $\bar{u} \in H$ is a weak solution of $-\Delta \bar{u}+a \bar{u}=\lambda \bar{u}$ on $\Omega$, where $\|\bar{u}\|_{2}=1$ and $\lambda_{1}<\lambda \leq \lambda_{1}+\delta<$ $\lambda_{2}$. Then

(i) (say) $\bar{u}>0$ a.e.,

(ii) $\|\bar{u}\|_{\infty} \leq K(\Omega) \lambda^{i(n)}$,

(iii) $\left\|\bar{u}-\phi_{1}\right\|_{2} \leq \varepsilon(\delta)$,

(iv) $\int_{\Omega} a \bar{u}^{2} \leq \lambda-\lambda_{1} \leq \delta$,

(v) if $\mu>0$, then $m\{x \in \Omega: a(x) \geq \mu\} \leq \varepsilon(\delta)$.

Here $\varepsilon(\delta) \rightarrow 0$ as $\delta \rightarrow 0$, independent of $a$ and $\bar{u}$. Moveover,

(vi) for every $\varepsilon>0$, there exist $\delta>0, s>0$ and $T \subset \Omega$ measurable such that $\bar{u}(x) \geq s$ for $x \in T$ and $m(\Omega-T)<\varepsilon ; \delta$ and $s$ are independent of $a$ and $\bar{u}$.

Proof. Let $\mu_{j}$ [resp., $\lambda_{j}$ ] be the eigenvalues of $-\Delta+a I$ [resp., $-\Delta$ ]. From the minimax characterization of eigenvalues [De, pp. 349-350] (see also [III, (1.2)] $\lambda_{j} \leq \mu_{j}(j=1,2, \ldots)$. Since $\lambda$ is an eigenvalue of $-\Delta+a I$ with eigenvector $\bar{u}$, and $\lambda_{1}<\lambda<\lambda_{2}, \lambda=\mu_{1}$. Thus (i) $\bar{u}>0$ a.e. or $<0$ a.e. (see [III, (1.6)]).

Conclusions (ii) and (iii) are (5.1) (see the sentence following it) and (5.4), respectively, and (iv) follows from the argument of (5.2) (2).

Let $\varepsilon>0$. Since $\phi_{1}(x)>0$ for $x \in \Omega$ [GT, Theorem (8.38), p. 214] and $\phi_{1}$ is continuous [GT, (8.11), p. 18], (1) there are a compact subset $X \subset \Omega$ and $r>0$ such that $m(\Omega-X) \leq \varepsilon / 3$ and $\phi_{1} \geq r$ on $X$. If $\delta$ is sufficiently small, then $\left\|\bar{u}-\phi_{1}\right\|_{2} \leq r \varepsilon^{1 / 2} / 4$ by (iii); on the other hand

$$
\left\|\bar{u}-\phi_{1}\right\|_{2}^{2}=\int_{\Omega}\left(\bar{u}-\phi_{1}\right)^{2} \geq m(Y) r^{2} / 4,
$$

where $Y=\left\{x \in \Omega:\left|\bar{u}-\phi_{1}\right| \geq r / 2\right\}$. This is (vi) with $T=X-Y$ and $s=r / 2$. 
Hence $m(Y) \leq \varepsilon / 4$. Thus, if $\widetilde{X}=\left\{x \in X:\left|\bar{u}-\phi_{1}\right| \leq r / 2\right\}$, then $m(\Omega-\widetilde{X}) \leq$ $2 \varepsilon / 3$ by (1) and $\bar{u} \geq r / 2$ on $\widetilde{X}$ by (1); hence $\int_{\Omega} a \bar{u}^{2} \geq\left(r^{2} / 4\right) \int_{\widetilde{X}} a$ and (2) $\int_{\widetilde{X}} a \leq 4 r^{-2} \delta$ by (iv). Now $\int_{\widetilde{X}} a \geq \mu m(Z)$, where $Z=\{x \in \widetilde{X}: a(x) \geq \mu\}$, and by (2), if $\delta$ is sufficiently small, $m(Z) \leq \varepsilon / 3$. Thus

$$
m\{x \in \Omega: a(x) \geq \mu\} \leq m(Z \cup(\Omega-\tilde{X})) \leq \varepsilon,
$$

which is $(\mathrm{v})$.

Here $K(\Omega)$ and $\varepsilon(\delta)$ are positive constants depending on $\Omega$ and $\delta$ respectively, and $i(n)=1,2, \ldots$ depends on $n$.

6.2. Lemma. Define $F(y, z)=(f(y)-f(z)) /(y-z)$ for $y \neq z$ and $=f^{\prime}(y)$ for $y=z$. Then

(i) given $\varepsilon_{1}>0$, there exists $\delta_{1}>0$ such that $F(y, z) \geq \delta_{1}$ on $\mathbb{R}^{2}-$ $\left\{(y, z):|y| \leq \varepsilon_{1}\right.$ and $\left.|z| \leq \varepsilon_{1}\right\}$ and

(ii) $F(y, z)>0$ for $(y, z) \neq(0,0)$ and $F(0,0)=0$.

Proof. Let $\delta_{1}=\eta \varepsilon_{1}{ }^{2} / 8$. From hypotheses (1.10), for $s \in \mathbb{R}$

$$
(\eta /(3-j) !)|s|^{3-j} \leq(\operatorname{sign} s)^{j+1} f^{(j)}(s) \leq\left(\left\|f^{(3)}\right\|_{\infty} /(3-j) !\right)|s|^{3-j}
$$

$(j=0,1,2,3)$, where $\operatorname{sign} s=1,-1$, or 0 according as $s>0,<0$, or $=0$, and thus for $y$ and $z$ of opposite sign and $\max \{|y|,|z|\} \geq \varepsilon_{1}$,

$$
F(y, z) \geq(\eta / 3 !)\left(|y|^{3}+|z|^{3}\right) /(|y|+|z|) \geq(\eta / 3 !)\left(y^{2}+y z+z^{2}\right) \geq \eta \varepsilon_{1}{ }^{2} / 8 .
$$

For $0 \leq z \leq y$ [resp., $y \leq z \leq 0$ ] and $|y| \geq \varepsilon_{1}$ and $\psi(z)=F(y, z), \psi^{\prime}(z)>0$ [resp., $<0$ ] from the Mean Value Theorem, and from (1)

$$
F(y, z) \geq F(y, 0)=f(y) / y \geq \eta y^{2} / 2 \geq \eta \varepsilon_{1}{ }^{2} / 2 .
$$

6.3. Lemma. Given $\varepsilon>0$, there exists $\delta>0$ such that, if $\lambda_{1}<\lambda<\lambda_{1}+\delta$ and if $u, v \in H$ with $u \neq v$ and $A_{\lambda}(u)=A_{\lambda}(v)$, then

(i) $u-v=\beta\left(\phi_{1}+w\right)$, where $w \in H_{1}$, and $u-v>0$ a.e. or $u-v<0$ a.e. on $\Omega$;

(ii) (a) $\|w\|_{2}<\varepsilon$ and (b) $|\beta|<\varepsilon$; and

(iii) $\left\|\phi_{1}+w\right\|_{\infty}<(1+\varepsilon) K(\Omega) \lambda^{i(n)}$.

Thus, as $\delta \rightarrow 0,\|u-v\|_{\infty} \rightarrow 0$, although for every $\delta>0, u \in S A$ need not be bounded in $H$, or even in $L^{1}(\Omega)$ [III, (2.6) and its proof].

Proof. By subtraction (1) $\bar{u}=\left(\|u-v\|_{2}{ }^{-1}(u-v)\right)$ solves $-\Delta \bar{u}+a \bar{u}=\lambda \bar{u}(6.1)$ with $a=F(u, v) \geq 0(6.2)$, and we may suppose that $\bar{u}>0$ a.e. (6.1)(i). Conclusion (i) with $\beta>0$ follows from (6.1)(i). Since

$$
\left\|\left(\phi_{1}+w\right)-\right\| \phi_{1}+w\left\|_{2} \phi_{1}\right\|_{2} \geq\|w\|_{2}
$$

and $\left\|\phi_{1}+w\right\|_{2}=\left[1+\|w\|_{2}^{2}\right]^{1 / 2}$, it follows from (i) that

$$
\left\|\bar{u}-\phi_{1}\right\|_{2} \geq\|w\|_{2}\left[1+\|w\|_{2}^{2}\right]^{-1 / 2}
$$

and conclusion (ii)(a) follows from (6.1)(iii). From (6.1)(ii)

$$
\|u-v\|_{\infty} \leq\|u-v\|_{2} K(\Omega) \lambda^{i(n)},
$$

and conclusion (iii) results from (i) and (ii)(a). 
We wish to prove (ii)(b). By $(6.1)(\mathrm{vi}),(2)$ there exist $\delta>0, s>0$ and $T$ measurable in $\Omega$ such that $m(\Omega-T)<m(\Omega) / 2$ and $\bar{u}(x) \geq s$ for $x \in T$. (Here $m(\Omega-T)$ and $s$ do not depend on $\bar{u}$.) Let $\varepsilon>0$, let $\delta_{1}$ be given by (6.2) for $\varepsilon_{1}<s \varepsilon / 2$, and let $W=\left\{x \in \Omega: a(x) \geq \delta_{1}\right\}$. By (6.2), (3) $|u(x)| \leq \varepsilon_{1}$ and $|v(x)| \leq \varepsilon_{1}$ for all $x \in \Omega-W$, and by (6.1) (v), (4) $m(W)<m(\Omega) / 2$ for $\delta$ sufficiently small. From (2) $|u(x)-v(x)| \geq s\|u-v\|_{2}$ for all $x \in T$, while by (3) $|u(x)-v(x)| \leq 2 \varepsilon_{1}$ for all $x \in \Omega-W$. Since $T \cap(\Omega-W) \neq \varnothing$ by (2) and (4), $s\|u-v\|_{2} \leq 2 \varepsilon_{1}<s \varepsilon$. By (i) $0<\beta<\|u-v\|_{2}$, and conclusion (ii)(b) results.

6.4. Lemma. Assume $\left\|f^{(3)}\right\|_{\infty}<\infty$. Given $\varepsilon>0$ there exists $\delta>0$ such that if $\lambda_{1}<\lambda<\lambda_{1}+\delta, A_{\lambda}(u)=A_{\lambda}(v)$ with $u \neq v$ and $u \in S A_{\lambda}$, and $e \in \operatorname{ker} D A_{\lambda}(u)$ with $\|e\|_{2}=1$ and $e>0$ a.e. [III, (1.6)], then

(i) $J(u) \neq 0$, i.e. $u$ is not a cusp point of $A_{\lambda}$; and

(ii) if $J(u)>0$ [resp., $J(u)<0]$, then $v<u$ a.e. and so $\int_{\Omega}(v-u) e<0$ [resp., $v>u$ a.e. and so $\int_{\Omega}(v-u) e>0$ ]. Moreover,

(iii) $v-u=\beta(e+\bar{w})$, where $\bar{w}$ is $L^{2}(\Omega)$-orthogonal to $e$;

(iv) (a) $|\beta|<\varepsilon$, (b) $\|\bar{w}\|_{2} \leq \varepsilon|\beta|$ and (c) $\|\bar{w}\|_{H} \leq C(\Omega) \lambda^{j(n)}|\beta|$; and

(v) $\|e+\bar{w}\|_{\infty}<(1+\varepsilon) K(\Omega) \lambda^{i(n)}$.

Proof. As $\delta \rightarrow 0,\|w\|_{2} \rightarrow 0$ by $(6.3)(\mathrm{ii})(\mathrm{a})$ and $\phi_{1}+w=\mu(e+\bar{w})$, where $\bar{w}$ is $L^{2}(\Omega)$-orthogonal to $e,(1)\|\bar{w}\|_{2} \rightarrow 0$ and $\mu \rightarrow 1$ by (6.1)(iii) (and (6.2) (ii)). Conclusions (iii), (iv)(a) and (v) follow from (6.3)(i), (ii)(b) and (ii).

Let $X=v-u$. From $A_{\lambda}(u)=A_{\lambda}(v)$ and Taylor's formula [Z, pp. 148-149],

$$
-\Delta X+\left(f^{\prime}(u)-\lambda\right) X=-\left(f^{\prime \prime}(u) / 2\right)(v-u)^{2}-h(u, v)(v-u)^{3},
$$

where

$$
\eta / 3 ! \leq h(u, v) \leq\left\|f^{(3)}\right\|_{\infty} / 3 !
$$

(6.2)(1), and since $D A_{\lambda}(u) \cdot e=0$,

$$
-\Delta \bar{w}+\left(f^{\prime}(u)-\lambda\right) \bar{w}=-\beta\left(f^{\prime \prime}(u) / 2\right)(e+\bar{w})^{2}-\beta^{2} h(u, v)(e+\bar{w})^{3} .
$$

Multiply by $\bar{w}$ and integrate to obtain

$$
\begin{aligned}
& \int_{\Omega}|\nabla \bar{w}|^{2}-\lambda_{1} \int_{\Omega} \bar{w}^{2}+\int_{\Omega} f^{\prime}(u) \bar{w}^{2} \\
& \quad=\left(\lambda-\lambda_{1}\right)\|\bar{w}\|_{2}{ }^{2}-\frac{\beta}{2} \int_{\Omega} f^{\prime \prime}(u)(e+\bar{w})^{2} \bar{w}-\beta^{2} \int_{\Omega} h(u, v)(e+\bar{w})^{3} \bar{w} .
\end{aligned}
$$

From $\int_{\Omega}|\nabla \bar{w}|^{2}-\lambda_{1} \int_{\Omega} \bar{w}^{2} \geq 0[\mathrm{GT},(8.94)$, p. 218], conclusion (v), (2), and $(6.2)(1)$,

$$
(\eta / 2) \int_{\Omega} u^{2} \bar{w}^{2} \leq\left(\lambda-\lambda_{1}\right)\|\bar{w}\|_{2}{ }^{2}+|\beta| K_{1}\|u \bar{w}\|_{2}+\beta^{2} K_{2}\|\bar{w}\|_{2},
$$

where $K_{j}=K_{j}(\Omega) \lambda^{i(n)}\left\|f^{(3)}\right\|_{\infty}(j=1,2)$. For $\delta=\lambda-\lambda_{1} \rightarrow 0,\|\bar{w}\|_{2} \leq 1$ by (1) and (by conclusion (iv)(a)) (6) $\|u \bar{w}\|_{2} \rightarrow 0$.

If $\Phi: \tilde{L} \rightarrow \tilde{H}$ is as defined in the proof of (5.2) and $g$ is the right side of (3), then $\bar{w}=\Phi g$ and $\|\Phi g\|_{2} \leq\left(\lambda_{2}(u)-\lambda\right)^{-1}\|g\|_{2}$. Since $\lambda_{2}(u) \geq \lambda_{2}$ [II, (2.3)] and $\lambda=\lambda_{1}+\delta \rightarrow \lambda_{1}$,

$$
\|\bar{w}\|_{2} \leq 2\left(\lambda_{2}-\lambda_{1}\right)^{-1}|\beta|^{-1}\left\|\left(f^{\prime \prime}(u) / 2\right)(v-u)^{2}+h(u, v)(v-u)^{3}\right\|_{2} .
$$


From conclusions (iii) and (v), (2), (5.1) and (6.2)(1),

$$
\begin{aligned}
\|\bar{w}\|_{2} \leq\left(\lambda_{2}-\lambda_{1}\right)^{-1}|\beta|\left\|f^{(3)}\right\|_{\infty}\left[\|u e\|_{2}\|e\|_{\infty}\right. & +2\|u \bar{w}\|_{2}\|e\|_{\infty}+\|u \bar{w}\|_{2}\|\bar{w}\|_{\infty} \\
& \left.+\left(|\beta|(m(\Omega))^{1 / 2} / 3\right)\|e+\bar{w}\|_{\infty}^{3}\right] .
\end{aligned}
$$

For $\delta$ sufficiently small, conclusion (iv)(b) follows from (6), (6.1)(iv) and conclusion (iv)(a), and (7) $\|u \bar{w}\|_{2} \leq C_{1}(\Omega) \lambda^{k(n)}\left\|f^{(3)}\right\|_{\infty}|\beta|$ follows from (5). Conclusion (iv)(c) follows from (4), (iv)(b), (v)(6.2)(1) and (7).

Suppose for $\delta$ sufficiently small, that $(8) J(u)=\int_{\Omega} f^{\prime \prime}(u) e^{3} \geq 0$ and $\beta>0$. Then

$$
\int f^{\prime \prime}(u) e(e+\bar{w})^{2} \geq 2 \int_{\Omega} f^{\prime \prime}(u) e^{2} \bar{w}+\int_{\Omega} f^{\prime \prime}(u) e \bar{w}^{2},
$$

while

$$
\left|\int_{\Omega} f^{\prime \prime}(u) e^{2} \bar{w}\right| \leq\left\|f^{(3)}\right\|_{\infty}\|e\|_{\infty}\|u e\|_{2}\|\bar{w}\|_{2} \leq \varepsilon \beta
$$

by (5.1), (6.1)(iv), conclusion (iv)(b) and (6.2)(1) and

$$
\left|\int_{\Omega} f^{\prime \prime}(u) e \bar{w}^{2}\right| \leq\left\|f^{(3)}\right\|_{\infty}\|\bar{w}\|_{\infty}\|u e\|_{2}\|\bar{w}\|_{2} \leq \varepsilon \beta
$$

by conclusions (v) and (iv)(b), (5.1), (6.1)(iv) and (6.2)(1). Thus

(9) $\int_{\Omega} f^{\prime \prime}(u) e(e+\bar{w})^{2} \geq-\varepsilon \beta$. By [A, Theorem 2.8, p. 25] and (2),

$$
\int_{\Omega} h(u, v) e^{4} \geq \eta(m(\Omega))^{-1} / 3 !,
$$

while from (5.1), conclusions (v) and (iv)(b), and (2),

$$
\int_{\Omega} h(u, v) e(e+\bar{w})^{3} \rightarrow \int_{\Omega} h(u, v) e^{4}
$$

as $\delta \rightarrow 0$, so that

$$
\int_{\Omega} h(u, v) e(e+\bar{w})^{3} \geq \eta(m(\Omega))^{-1} / 12 .
$$

In (3) multiply by $e$ and integrate to obtain

$$
\int_{\Omega} f^{\prime \prime}(u) e(e+\bar{w})^{2}+2 \beta \int_{\Omega} h(u, v) e(e+\bar{w})^{3}=0,
$$

and from (8), (9) and (10) a contradiction results. Thus (8) is false.

Similarly, suppose for $\delta$ sufficiently small that $\left(8^{\prime}\right) \int_{\Omega} f^{\prime \prime}(u) e^{3} \leq 0$ and $\beta<$ 0 . The integral in (9) is bounded above by $\varepsilon|\beta|$ and from (11) a contradiction of $\left(8^{\prime}\right)$ results.

By conclusion (iii), $0 \neq \beta=\int_{\Omega}(v-u) e$, and conclusions (i) and (ii) result from (6.3)(i).

It also follows from the falsity of (8) and (8') that, if $A_{\lambda}^{-1}(y) \cap S A_{\lambda}$ for some $y \in H$ has at least two points, then it has precisely two, $u$ and $v$, with $J(u)>0$ and $J(v)<0$. In (6.5) we prove that each $A_{\lambda}{ }^{-1}(y) \cap S A_{\lambda}$ has at most one point.

6.5. Proposition. Assume $\left\|f^{(3)}\right\|_{\infty}<\infty$. The restriction map $A_{\lambda} \mid S A_{\lambda}$ is injective for $\lambda_{1}<\lambda<\lambda_{1}+\delta$ with $\delta$ sufficiently small.

Proof. Suppose the contrary, i.e., there exist $u, v \in S A_{\lambda}$ with $A_{\lambda}(u)=A_{\lambda}(v)$, $u \neq v$, and $\delta$ small. Let $e \in \operatorname{ker} D A_{\lambda}(u)$ and $\bar{e} \in \operatorname{ker} D A_{\lambda}(v)$ with $\|e\|_{2}=$ $\|\bar{e}\|_{2}=1$ and $e(x)>0$ and $\bar{e}(x)>0$ a.e. on $\Omega$. By (6.1)(iii), (1) $\bar{e}=e+y$, 
where $\|y\|_{2} \rightarrow 0$ as $\delta \rightarrow 0$. From $-\Delta \bar{e}+\left(f^{\prime}(v)-\lambda\right) \bar{e}=0$ and Taylor's formula [Z, pp. 148-149],

$-\Delta \bar{e}+\left(f^{\prime}(u)-\lambda\right) \bar{e}=\left(f^{\prime}(u)-f^{\prime}(v)\right) \bar{e}=-\left(f^{\prime \prime}(u)(v-u)+g(u, v)(v-u)^{2}\right) \bar{e}$, where (2) $\eta / 2 \leq g(u, v) \leq\left\|f^{(3)}\right\|_{\infty} / 2$, and by (1) and (6.4)(iii)

$$
-\Delta y+\left(f^{\prime}(u)-\lambda\right) y=-\beta f^{\prime \prime}(u)(e+\bar{w}) \bar{e}-\beta^{2} g(u, v)(e+\bar{w})^{2} \bar{e} .
$$

Modulo factors bounded by $C(\Omega) \lambda^{j(n)}\left\|f^{(3)}\right\|_{\infty}$ on the right side and the use of (3), equation (3) is the same as (6.4)(3) with $\bar{w}$ replaced by $y$ on the left, and the argument of (6.4)(3)-(7) yields the analogs of (6.4)(iv)(b) and (7) with $\bar{w}$ replaced by $y$, viz. (4) $\|y\|_{2} \leq \varepsilon|\beta|$ and (5) $\|u y\|_{2} \leq C(\Omega) \lambda^{j(n)}\left\|f^{(3)}\right\|_{\infty}|\beta|$ for $\delta$ small.

Multiply (3) by $e$, integrate and use (1) to obtain

$$
\int_{\Omega} f^{\prime \prime}(u) e^{3}+\int_{\Omega} f^{\prime \prime}(u) \bar{w} e^{2}+\int_{\Omega} f^{\prime \prime}(u)(e+\bar{w}) e y+\beta \int_{\Omega} g(u, v)(e+\bar{w})^{2} \bar{e} e=0 .
$$

By (5), (6.1)(ii) and (iv), and (6.4)(iv)(b) and (v), (6.2)(1) the second and third terms in magnitude are less than $\varepsilon|\beta|$ for $\delta$ small, and by (1), (4), (5.1) and $(6.4)(\mathrm{iv})(\mathrm{b})$

$$
\left|\int_{\Omega} g(u, v)(e+\bar{w})^{2} e \bar{e}-\int_{\Omega} g(u, v) e^{4}\right|<\varepsilon .
$$

By $[A$, Theorem 2.8, p. 25] and (2),

$$
\int_{\Omega} g(u, v) e^{4} \geq \eta(m(\Omega))^{-1} / 2
$$

so that

$$
\int_{\Omega} f^{\prime \prime}(u) e^{3}=-\beta(1+\mu) \int_{\Omega} g(u, v) e^{4},
$$

where $\mu \rightarrow 0$ as $\delta \rightarrow 0$. From (11) in the proof of (6.4) and from (2) and an argument similar to that for $(5)$,

$$
\int_{\Omega} f^{\prime \prime}(u) e^{3}=-\beta(1+\nu) \int_{\Omega} 2 h(u, v) e^{4},
$$

where $\nu \rightarrow 0$ as $\delta \rightarrow 0$. By (6.4)(i) $\int_{\Omega} f^{\prime \prime}(u) e^{3} \neq 0$, and division of (5) by (6) yields

$$
\left(\int_{\Omega}(g(u, v)-2 h(u, v)) e^{4}\right) / \int_{\Omega} 2 h(u, v) e^{4} \rightarrow 0
$$

as $\delta \rightarrow 0$.

From Taylor's Formula [Z, pp. 148-149]

$$
\int_{0}^{1}(1-\tau)^{i} f^{(3)}(u(x)+\tau(v(x)-u(x))) d \tau
$$

is $g(u(x), v(x))$ for $i=1$ and $2 h(u(x), v(x))$ for $i=2$. Let $\psi(\tau)=$ $(1-\tau)-(1-\tau)^{2} \geq 0$, so that $\psi(t) \geq 2 / 9$ for $1 / 3 \leq \tau \leq 2 / 3$. From (6.4)(2) and (1.10)(iii), (7) is bounded below by

$$
\left(\alpha+\frac{\eta}{9} \int_{\Omega} e^{4}\right) /\left(\left\|f^{(3)}\right\|_{\infty} \int_{\Omega} e^{4}\right)
$$

where $\alpha \geq 0$, and a contradiction of (7) results. 


\section{THE CUSP SET IS HOMEOMORPHIC TO $H$}

An integral curve argument is used to obtain the first main result (7.2) of this section: there exists $\bar{\lambda}>\lambda_{1}$ such that for $\lambda \in(-\infty, \bar{\lambda}),(S A, C A)$ is $C^{k-1}$ (real analytic if $f(u)=u^{3}$ ) diffeomorphic to $(H, \Gamma)$, where $\Gamma$ is a codimension one subspace of $H$. A technical lemma (7.1) is required. The second main result is (7.9) and (7.11): for $\left\|f^{(3)}\right\|_{\infty}<\infty$ there exists $\bar{\lambda}>\lambda_{1}$ such that for $\lambda_{1}<\lambda<\bar{\lambda},\left(S A_{\lambda}, C A_{\lambda}\right)$ is homeomorphic $\left(C^{\infty}\right.$ diffeomorphic if $f$ is $C^{\infty}$ in the Sobolev case) to $(H, \Gamma)$ also. As usual we actually assume hypotheses (1.10).

In (4.2) $C A$ is defined to be the set of precusp points, while for some $\bar{\lambda}>\lambda_{1}$ and all $\lambda<\bar{\lambda}$, all precusp points are cusp points (5.2). Thus in this section (and in $\S 8) C A$ stands for the set of cusp points (1.5).

\subsection{Lemma. Let $e: H \rightarrow H$ satisfy [III, (1.9)]}

$$
\left.\int_{\Omega}|\nabla e(u)|^{2}-\lambda_{1}(u) \int_{\Omega} e(u)\right)^{2}+\int_{\Omega} f^{\prime}(u)(e(u))^{2}=0,
$$

normalized with $1=\|e\|_{H}^{2}=\int_{\Omega}|\nabla e(u)|^{2}$, and let $u: \mathbb{R} \rightarrow H$ be Gâteaux differentiable and satisfy (ii) $u^{\prime}(t)=e(u(t))$. Then

$$
\limsup _{t \rightarrow \infty} \lambda_{1}(u(t))=\limsup _{t \rightarrow \infty} \lambda_{1}(u(-t))=\infty .
$$

Proof. Suppose the contrary. Then (1) there exist $\alpha \in \mathbb{R}$ and $K>0$ such that $\lambda_{1}(u(t)) \leq K$ [resp., $\lambda_{1}(u(-t)) \leq K$ ] for $\alpha \leq t<\infty$. Hereafter, for the respective version, just replace $u(t)$ by $-u(-t)$ (and $u(j)$ by $-u(-j)$ ). (2) Let $E$ be the set of all $e=e(u)$ with $\lambda_{1}(u) \leq K$; from the hypothesis (i) $1 / K \leq\|e\|_{2}{ }^{2}$ and from (5.1) there exists $M>0$ with $\|e\|_{\infty} \leq M$ for all $e \in E$. If $A_{n}=\{x \in \Omega: e(x) \geq 1 / n\}$, then [III, (1.6)]

$$
\frac{1}{K} \leq \int_{\Omega} e^{2} \leq \frac{m\left(\Omega-A_{n}\right)}{n^{2}}+M^{2} m\left(A_{n}\right) ;
$$

thus (3) there exist $\tau, 0<\tau<m(\Omega) / 4$, and $\mu>0$ such that

$$
m\{x: e(x) \geq \mu\} \geq \tau \text { for all } e \in E .
$$

Let $\delta=\tau \mu / 2(m(\Omega))^{1 / 2}$; since $E$ has compact closure in $L^{2}(\Omega)$ [A, pp. 143-144] and hypothesis (i) there exist $e_{i} \in E(i=1,2, \ldots, r) \delta$-dense in $E$ (in $L^{2}(\Omega)$ ). (4) If $T_{i}$ is the set of $t, \alpha \leq t<\infty$, such that $\left\|u^{\prime}(t)-e_{i}\right\|_{2} \leq \delta$, then $[\alpha, \infty)=\bigcup\left\{T_{i}: i=1,2, \ldots, r\right\}$ by hypothesis (ii). Hence, (5) for some $i, T_{i}$ has infinite measure; hereafter we consider this $i$. By Cauchy-Schwarz (6) $\left\|u^{\prime}(t)-e_{i}\right\|_{1} \leq m(\Omega)^{1 / 2} \delta$ for $t \in T_{i}$.

Let $V_{i}=\left\{x \in \Omega: e_{i}(x) \geq \mu\right\}$; then (7) $m\left(V_{i}\right) \geq \tau$ by (3). Let $W_{n}=\left\{x \in V_{i}\right.$ : for some $t \in \mathbb{R}, u(t)(x) \geq n\}(n=1,2, \ldots)$, so that

$$
\int_{V_{i}-W_{n}} u(t) \leq n m\left(V_{i}-W_{n}\right)
$$

for all $t \in \mathbb{R}$. (9) For each fixed $x \in \Omega, u(t)(x)$ is an increasing function of $t$ by hypothesis (ii) and [III, (1.6)]. For $t \in T_{i}$,

$$
\frac{d}{d t} \int_{V_{i}-W_{n}} u(t)=\int_{V_{i}-W_{n}} u^{\prime}(t) \geq \mu m\left(V_{i}-W_{n}\right)-\delta m(\Omega)^{1 / 2},
$$


by (6), (9) and definition of $V_{i}$, and by (5), (9) and (8), $m\left(V_{i}-W_{n}\right) \leq$ $\delta m(\Omega)^{1 / 2} / \mu=\tau / 2$. Thus (by (7)) (10) $m\left(W_{n}\right) \geq \tau / 2$.

By (1), hypotheses (i) and (1.10), and [A, p. 97], (11) for some $C>0$

$$
(\eta / 2) \int_{W_{n}}(u(t))^{2}(e(u(t)))^{2}<\int_{W_{n}} f^{\prime}(u(t))(e(u(t)))^{2}<C
$$

for all $t \geq \alpha$. If $\tilde{W}_{j}=\left\{x \in W_{n}: u(j)(x) \geq n\right\}$, then $\tilde{W}_{j-1} \subset \tilde{W}_{j}$ by (9) and $W_{n}=\bigcup_{j} \tilde{W}_{j}$; thus, for $j$ sufficiently large, (12) $m\left(\tilde{W}_{j}\right) \geq(2 / 3) m\left(W_{n}\right) \geq \tau / 3$ by (10). If $t \geq j \geq \alpha$, then

$$
n^{2} \int_{\tilde{W}_{j}}(e(u(t)))^{2} \leq \int_{\tilde{W}_{j}}(u(t))^{2}(e(u(t)))^{2} \leq 2 C / \eta
$$

by (9) and (11). Since $n$ may be arbitrarily large, (13) there exists $X_{t} \subset \tilde{W}_{j}$ such that $m\left(X_{t}\right) \geq \tau / 4$ and (by (12)) $e(u(t))(x)<\mu / 2$ for $x \in X_{t}$.

On the other hand, if $t \in T_{i}$, then, by (3), (4) and hypothesis (ii), $\mid e(u(t))-$ $e_{i} \mid \geq \mu / 2$ on a subset $Y \subset V_{i}$ of measure at most $4 \delta^{2} / \mu^{2}=\tau^{2} / m(\Omega)<\tau / 4$. A contradiction results from (5), (13) and the definition of $V_{i}$.

7.2. Proposition. Let $A$ be a $C^{k}(k=3,4, \ldots$ or $\infty)$ map satisfying (1.10) with $\left\|f^{(3)}\right\|_{\infty}<\infty$. Let $\bar{\lambda}>\lambda_{1}$ be any real number (or $\left.\infty\right)$ given by the conclusion of (5.2), i.e. $A$ has only folds or positive cusps for $\lambda<\bar{\lambda}$, let $U(\bar{\lambda})=\left\{u \in H: \lambda_{1}(u)<\bar{\lambda}\right\}$, and let $C$ be the set of cusp points of $A$ for $\lambda<\bar{\lambda}$. Then there is a $C^{k-1}$ diffeomorphism (real analytic if $f(u)=u^{3}$ ) $\Phi$ of $U(\bar{\lambda})$ onto an open set in $C \times \mathbb{R}$ such that (i) $\Phi(c)=(c, 0)$ for each $c \in C$ and (ii) $D \phi(u) \cdot e=(0,1)$ for each $u \in U(\bar{\lambda})$ and $e$ normalized by $\|e\|_{H}=1$. Moreover (iii) for $c \in C$ the set $\Gamma_{c}=\{t \in \mathbb{R}:(c, t) \in \Phi(U(\bar{\lambda}))\}$ is an open interval containing 0 , (iv) the map $\mu_{1}: \Gamma_{c} \rightarrow \mathbb{R}$ defined by $\mu_{1}(t)=\lambda_{1} \Phi^{-1}(c, t)$ has 0 as its only critical point and (v) has its absolute minimum there. Thus $J\left(\Phi^{-1}(c, t)\right)$ has the same sign as $t$, where $J(u)=\int_{\Omega} f^{\prime \prime}(u) e^{3}$.

Proof. If $k=\infty$ [resp., $f(u)=u^{3}$ ] interpret $C^{k-1}$ as $C^{\infty}$ [resp., real analytic]. First assume that $\bar{\lambda}<\infty$. Consider the $C^{k-1}$ [III, (1.9)] vector field $e: U(\bar{\lambda}) \rightarrow H$. It follows from $\lambda_{1}: U(\bar{\lambda}) \rightarrow \mathbb{R}$ being $C^{k-1}$ [III, (1.8); L, Corollary 3, p. 70], and (7.1) that (1) each integral curve $\gamma$ in $U(\bar{\lambda})$ can be defined on a bounded interval $\left(a_{0}, b_{0}\right)$ such that the limits as $t \rightarrow a_{0}$ and $t \rightarrow b_{0}$ of $\lambda_{1}$ on this curve are $\bar{\lambda}$. Since $\bar{\lambda} \in \mathbb{R}$ is arbitrary, the analogous result for $\bar{\lambda}=\infty$ follows from the uniqueness of integral curves (e.g. [L, Corollary 2, p. 69]).

For each integral curve $\gamma$, the critical points of $\lambda_{1} \gamma$ occur at the cusp points by $(5.3)(\mathrm{b})$ and (3.1), and each is a local minimum by (5.3)(c) and (5.2); thus (from (1)) (2) $\lambda_{1} \gamma$ has exactly one critical point $c$, its absolute minimum, taken at a cusp point. Moreover, from (5.3)(c) and (5.2), (3) $J \gamma>0$ on one side of $C$ and negative on the other.

Let $\alpha: D \rightarrow U(\lambda)$ be the resulting $C^{k-1}$ flow with domain $D[\mathrm{~L}, \mathrm{pp} .77-$ 80]. (Consider $f(u)=u^{3}$. Since $e: U(\bar{\lambda}) \rightarrow H$ is real analytic [III, (1.9)], it has a local complexification $\tilde{e}$ and resulting complex analytic flow $\tilde{\alpha}$ [D, (7.4.2), p. 283]. Thus $\alpha$ is real analytic.) For each $t \in \mathbb{R}, \alpha \mid(D \cap(U(\bar{\lambda}) \times t))$ is a local diffeomorphism, so if $C$ is the cusp set $C(A \mid U(\bar{\lambda})), \alpha \mid(D \cap(C \times t))$ is an immersion; since $e=e(u)$ is transverse to $C$ at $u(5.2), \beta=\alpha \mid(D \cap(C \times \mathbb{R}))$ 
is a local diffeomorphism, and (4) $\beta(c, 0)=c$ for each $c \in C$. From (1), (2) and (3) the restriction of $\beta$ to each integral curve is injective, so that $\beta$ is injective, and since each point of $U(\bar{\lambda})$ lies on an integral curve meeting $C$ by (2), $\beta$ maps onto $U(\bar{\lambda})$. Thus $\beta:(D \cap(C \times \mathbb{R})) \rightarrow U(\bar{\lambda})$ is a $C^{k-1}$ diffeomorphism (onto); then its inverse $\Phi$ satisfies the desired properties. The last conclusion results from $(5.3)(\mathrm{b})$ and (c).

7.3. Remark. The analogous result for the closure of $U(\bar{\lambda})$ follows readily from substantially the same proof.

7.4. Corollary. Let $A$ be a $C^{k}(k=3,4, \ldots$ or $\infty)$ map satisfying (1.10) with $\left\|f^{(3)}\right\|_{\infty}<\infty$. Assume only folds and positive cusps for $\lambda \leq \bar{\lambda}<\lambda_{2}$. Let $F_{+}\left[\right.$resp., $\left.F_{-}\right]$be the set of $u \in S A_{\bar{\lambda}}$ with $J(u)>0$ [resp., $\left.<0\right]$, let $C$ be the cusp set of $A \mid U(\bar{\lambda})$, and let $C^{k-1}$ [resp., $C^{0}$ ] diffeomorphism be denoted by $\approx_{k-1}\left[\right.$ resp., $\left.\approx_{0}\right]$. Then

(i) $(U(\bar{\lambda}), C) \approx_{0}\left(H, H_{1}\right)$, where $H_{1}$ is a codimension one subspace of $H$;

(ii) $\left(\bar{F}_{+}, C A_{\bar{\lambda}}\right) \approx_{0}\left(\bar{F}_{-}, C A_{\bar{\lambda}}\right) \approx_{0}\left(\bar{C}, C A_{\bar{\lambda}}\right)$ with $F_{+} \approx_{k-1} F_{-} \approx_{k-1} C$; and

(iii) $C A_{\bar{\lambda}}$ is Z-acyclic.

In case $f(u)=u^{3}$, the diffeomorphisms $F_{+} \approx F_{-} \approx C^{\prime}$ in (ii) are real analytic, and that in (i) is $C^{\infty}$. More generally, if $S A$ and its subsets are replaced by the graph of $\lambda_{1}: H \rightarrow \mathbb{R}$ and its subsets, the restriction $\bar{\lambda}<\lambda_{2}$ can be omitted. The ring of integers is $Z$, and a set is $Z$-acyclic if it has the singular homology using $Z$-coefficients of a point.

Proof. Since $U(\bar{\lambda})$ is star-shaped (see (3.6)), it is contractible. Since $e$ is transverse to $S A_{\bar{\lambda}}$ at each point of $F_{+} \cup F_{-}$, i.e. of $S A_{\bar{\lambda}}-C A_{\bar{\lambda}}$, by $(5.3(\mathrm{~b}))$, it follows from $(7.2)$ that $(1)(U(\bar{\lambda}), C) \approx_{k-1}(C \times(-1,1), C \times 0)$. Thus $C$ is also contractible, and $C \approx_{k-1} H_{1}$, by [K-2, Theorem 7.3, p. 106]. Conclusion (i) follows from [K-1, Corollary 6, p. 89] or [BH, Theorem 0.1, p. 1261].

From (1) and (7.3) there are $C^{k-1}$ diffeomorphisms $\psi_{+}: F_{+} \approx_{k-1} C$ and $\psi_{-}: F_{-} \approx_{k-1} C$, and bdy $F_{+}=$bdy $F_{-}=$bdy $C=C A_{\lambda}$. At each point of $C A_{\lambda}$ it follows from the argument of (7.2) that there is locally the structure of (1). As a result $\psi_{+}$and $\psi_{-}$can be extended to $\bar{\psi}_{+}: \bar{F}_{+} \approx_{0} \bar{C}$ and $\psi_{-}: \bar{F}_{-} \approx_{0} \bar{C}$ with $\bar{\psi}_{+}(c)=\bar{\psi}_{-}(c)=c$ for each $c \in C A_{\bar{\lambda}}$. This is conclusion (ii).

Conclusion (iii) follows from (3.9), (3.13) conclusion (i), conclusion (ii) and the Mayer-Vietoris sequence [Sp, pp. 186-190] applied to $S A_{\bar{\lambda}}, \bar{F}_{+}, \bar{F}_{-}$, and $C A_{\bar{\lambda}}$.

The remainder of $\S 6$ is devoted to the proof that, for $\lambda_{1}<\lambda<\lambda_{1}+\delta$ and $\delta>0$ sufficiently small, the cusp set $C A_{\lambda}$ is contractible $((7.9)$ and (7.11)) for $f(u)=u^{3}$. Remember $\left\|\phi_{1}\right\|_{2}=\|e\|_{2}=1((1.8)$ and (1.9)).

7.5. Lemma. If $\delta>0$ is sufficiently small, $\lambda<\lambda_{1}+\delta$, and $k \in H$ with $\int_{\Omega} k e=0$, then

$$
\int_{\Omega}\left[|\nabla k|^{2}-\lambda k^{2}\right] \geq C\|k\|_{2}^{2},
$$

where $C>0$ is a constant independent of $k, e$, and $\lambda$. 
Proof. Write $k=\beta \phi_{1}+w$, where $\int_{\Omega} w \phi_{1}=0$, so that

$$
\int_{\Omega}|\nabla w|^{2} \geq \lambda_{2} \int_{\Omega} w^{2}
$$

By homogeneity we may suppose that $\beta=1$, and thus

$$
\int_{\Omega}\left[|\nabla k|^{2}-\lambda k^{2}\right] \geq\left(\lambda_{2}-\lambda\right)\|w\|_{2}^{2}-\left(\lambda-\lambda_{1}\right) .
$$

Since $S A=\phi$ for $\lambda<\lambda_{1}$ ((2.5)(iii) and [II, (2.3)]), we may suppose $\lambda_{1} \leq \lambda<$ $\lambda_{1}+\delta$.

Suppose the conclusion is false; then there exist $\lambda(n) \geq \lambda_{1}$ with $\lambda(n)-\lambda_{1}<$ $1 / n, e_{n}=e\left(u_{n}, \lambda(n)\right)$, and $k_{n}$ with $(2) \int_{\Omega} k_{n} e_{n}=0$ and

$$
\int_{\Omega}\left[\left|\nabla k_{n}\right|^{2}-\lambda(n) k_{n}^{2}\right] \leq\left\|k_{n}\right\|_{2}^{2} / n
$$

By (1),

$$
\left(\left(\lambda_{2}-\lambda_{1}\right) / 2\right)\left\|w_{n}\right\|_{2}^{2}-\left(\lambda(n)-\lambda_{1}\right) \leq\left(1+\left\|w_{n}\right\|_{2}^{2}\right) / n,
$$

and it follows that $\left\|w_{n}\right\|_{2} \rightarrow 0$, that is $k_{n} \rightarrow \phi_{1}$ in $L^{2}(\Omega)$. From (5.4) $e_{n} \rightarrow \phi_{1}$ in $W_{0}^{1,2}(\Omega)$ and so in $L^{2}(\Omega)$ [A, p. 97], and thus

$$
\int_{\Omega} k_{n} e_{n}-\int_{\Omega} k_{n} \phi_{1} \rightarrow 0
$$

Since $\int_{\Omega} k_{n} \phi_{1}=1$, a contradiction results from (2).

The following definitions are used in the remainder of $\S 7$.

7.6. Definitions and Remarks. Assume $\left\|f^{(3)}\right\|_{\infty}<\infty$. Let $L=\left\{c \phi_{1}: c \in \mathbb{R}\right\}$. Since $e>0$ [III, (1.6)] and $\phi_{1}>0$ [GT, Theorem 8.38, p. 214] on $\Omega, J(u)=$ $\int_{\Omega} f^{\prime \prime}(u)(e(u))^{3} \neq 0$ if $u \in L-\{0\}$. For $\lambda_{1}<\lambda<\lambda_{2}, 0 \notin S A_{\lambda}$ [II, (2.6)], and by $(3.1)$

$$
C A_{\lambda} \subset S A_{\lambda}-L \text {. }
$$

Let $H_{1}=\left\{v \in H: \int_{\Omega} v \phi_{1}=0\right\}$ and write $w \in H-L$ as $v+t \phi_{1}$, where $v \in H_{1}-\{0\}$ and $t \in \mathbb{R}$. Define $\Gamma$ [resp., $\Lambda$ ] as the set of $v+t \phi_{1} \in H-L$ such that $\alpha \cdot\left(v+t \phi_{1}\right) \in S A_{\lambda}-L$ [resp., $C A_{\lambda}$ ] for some $\alpha>0$.

From [III, (2.5)] (see (3.6)) for each $v+t \phi_{1} \in \Gamma, \alpha$ is unique and from [III, (2.9)] (2) $\Gamma$ is open in $H-L$. Let $\alpha=\alpha\left(v+t \phi_{1}\right)=\alpha(t)$ and let $u(t)=\alpha \cdot\left(v+t \phi_{1}\right)$. Then (3) $\left(S A_{\lambda}, C A_{\lambda}\right)$ is a deformation retract of $(\Gamma, \Lambda)$, with retraction $\Psi\left(v+t \phi_{1}\right)=u(t)$.

(4) Now $e(u(t))$, call it $e=e(t)$, is $C^{k-1}$ (real analytic if $f(u)=u^{3}$ ) in $v+t \phi_{1}$ [III, Theorem 1.9, p. 204]; let $k(t)=d e / d t$. Since $\|e(t)\|_{2}=1$,

$$
\int_{\Omega} e(t) k(t)=0
$$

(5) Define a continuous (real analytic if $f(u)=u^{3}$ ) function $F: \Gamma \rightarrow \mathbb{R}$ by

$$
F\left(v+t \phi_{1}\right)=\int_{\Omega} f^{\prime \prime}(u(t))(e(t))^{3}=J(u(t)) .
$$

Thus $\Lambda$ is the set of zeros of $F$, where $\Psi(\Lambda)=C A_{\lambda}$, and $\partial F / \partial t$ is continuous: use (5.1), (1.10), the Hölder inequality [A, p. 23] and [Kr, Theorem 2.1, p. 22]. 
(6) The set $T=\left\{u \in H \cap L^{\infty}(\Omega): u\right.$ is real analytic $\}$ is convex and contained in $\Gamma \cup L$ by (3.7); in the Sobolev case $T-L$ is dense in the open set (2) $\Gamma$ by (3.8)(a) and in the Hölder case it is $L^{\infty}(\Omega)$-dense by (3.11).

7.7. Lemma. Assume $\left\|f^{(3)}\right\|_{\infty}<\infty$. There exist $\delta>0$ and $a>0$ such that, if $\lambda_{1}<\lambda<\lambda_{1}+\delta$, then

$$
(\alpha(t))^{-1}(\partial F / \partial t)\left(v+t \phi_{1}\right) \geq a
$$

for every $v+t \phi_{1} \in \Lambda$ (7.6). Thus $\partial F / \partial t>0$ on $\Lambda$.

Proof. Write $(\alpha(t))^{-1} \partial F / \partial t\left(v+t \phi_{1}\right)=A+B+C$, where

$$
\begin{aligned}
& A=3(\alpha(t))^{-1} \int_{\Omega} f^{\prime \prime}(u(t))(e(t))^{2} k(t), \\
& B=\int_{\Omega} f^{(3)}(u(t)) \phi_{1}(e(t))^{3},
\end{aligned}
$$

and

$$
C=\alpha^{\prime}(t)(\alpha(t))^{-2} \int_{\Omega} f^{(3)}(u(t)) u(t)(e(t))^{3} .
$$

By (1.10) $B \geq \eta \int_{\Omega} \phi_{1}(e(t))^{3}$ and by (5.4), (5.1) and [A, pp. 97 and 25] the latter integral converges to $\int_{\Omega} \phi_{1}^{4}=2 a$ as $\delta \rightarrow 0$. We will prove that (1) $A$ converges to 0 on $\Gamma$ as $\delta \rightarrow 0$ and (2) $C$ converges to 0 on $\Lambda$ as $\delta \rightarrow 0$; the desired conclusion will result.

For some $M>0,\|e(t)\|_{\infty} \leq M(5.1),\left|f^{\prime \prime}(u(t))\right| \leq\left\|f^{(3)}\right\|_{\infty}|u(t)|(1.10)$ and

$$
\|u(t) e(t)\|_{2}<\left[2\left(\lambda-\lambda_{1}\right) / \eta\right]^{1 / 2}
$$

$\left((5.2)(2)\right.$ and (1.10)), so for (1) it suffices to prove that (3) $\left\|(\alpha(t))^{-1} k(t)\right\|_{2}$ is bounded for $\delta>0$ sufficiently small.

Differentiate (7.6) (4)

$$
-\Delta e(t)+\left(f^{\prime}(u(t))-\lambda\right) e(t)=0
$$

to obtain

$$
\begin{aligned}
& -\Delta k(t)+\left(f^{\prime}(u(t))-\lambda\right) k(t) \\
& \quad=-f^{\prime \prime}(u(t)) \alpha(t) \phi_{1} e(t)-f^{\prime \prime}(u(t)) \alpha^{\prime}(t)\left(v+t \phi_{1}\right) e(t) .
\end{aligned}
$$

Multiply by $e(t)$ and use (4) and selfadjointness to derive

$$
\alpha^{\prime}(t) \int_{\Omega} f^{\prime \prime}(u(t))\left(v+t \phi_{1}\right)(e(t))^{2}=-\alpha(t) \int_{\Omega} f^{\prime \prime}(u(t)) \phi_{1}(e(t))^{2}
$$

and by (1.10)

$$
\left|\alpha^{\prime}(t)\right| \eta \alpha(t) \int_{\Omega}\left(v+t \phi_{1}\right)^{2}(e(t))^{2} \leq(\alpha(t))^{2}\left\|f^{(3)}\right\|_{\infty} \int_{\Omega}\left(v+t \phi_{1}\right) \phi_{1}(e(t))^{2} .
$$

By the Cauchy-Schwarz inequality, (5.4) and [A, p. 97]

$$
\begin{aligned}
(\alpha(t))^{-1}\left|\alpha^{\prime}(t)\right| & \leq \eta^{-1}\left\|f^{(3)}\right\|_{\infty}\left\|\phi_{1} e(t)\right\|_{2} /\left\|\left(v+t \phi_{1}\right) e(t)\right\|_{2} \\
& \leq K_{1}(f, \Omega) /\left\|\left(v+t \phi_{1}\right) e(t)\right\|_{2},
\end{aligned}
$$

where $K_{1}(f, \Omega)>0$ is a positive constant depending only on $f$ and $\Omega$. 
As in the second paragraph of the proof of (5.2) let $\tilde{L}$ and $\tilde{H}$ be the $L^{2}(\Omega)$ orthogonal complements of $e$ in $L^{2}(\Omega)$ and $H=W_{0}^{1,2}(\Omega)$, respectively, define $\Phi: \tilde{L} \rightarrow \tilde{H}$ by $\left\langle D A_{\lambda}(u) \cdot \Phi g, \psi\right\rangle_{H}=\langle g, \psi\rangle_{2}$ for every $\psi \in H$, and $P: L^{2}(\Omega) \rightarrow L^{2}(\Omega)$ as projection onto $\tilde{L}$. Since $\phi_{1}$ and $e$ are in $L^{\infty}(\Omega)$ ([GT, Theorem 8.15, p. 189] and (5.1)) we may [A, p. 97] define

$$
k_{1}(t)=\Phi\left(-P\left(\alpha(t) f^{\prime \prime}(u(t)) \phi_{1} e(t)\right)\right)
$$

(i.e. $\left.-\Delta k_{1}(t)+\left(f^{\prime}(u(t))-\lambda\right) k_{1}(t)=-P\left(\alpha(t) f^{\prime \prime}(u(t)) \phi_{1} e(t)\right)\right)$ and

$$
k_{2}(t)=\Phi\left(-P\left(\alpha^{\prime}(t) f^{\prime \prime}(u(t))\left(v+t \phi_{1}\right) e(t)\right)\right) .
$$

Thus (7) $k(t)=k_{1}(t)+k_{2}(t)$ by (5) and (7.6) (4).

As in the proof of (5.2)

$$
\begin{aligned}
\left\|k_{1}(t)\right\|_{2} & \leq\left(\lambda_{2}-\lambda\right)^{-1}\left\|P\left(\alpha(t) f^{\prime \prime}(u(t)) \phi_{1} e(t)\right)\right\|_{2} \\
& \leq K_{2}\|P\|\left\|\phi_{1}\right\|_{\infty} \alpha(t)\left\|\alpha(t)\left(v+t \phi_{1}\right) e(t)\right\|_{2} \\
& \leq K_{3} \alpha(t)
\end{aligned}
$$

by (5.2) (2) and (1.10). Thus (8) $\left\|(\alpha(t))^{-1} k_{1}(t)\right\|_{2}$ is uniformly bounded for $\lambda<\lambda_{2}$ (indeed, it is small for $\delta>0$ small).

Now

$$
\begin{aligned}
& \int_{\Omega}\left[\left|\nabla k_{2}(t)\right|^{2}+\left(f^{\prime}(u(t))-\lambda\right) k_{2}^{2}\right] \\
& =-\alpha^{\prime}(t) \int_{\Omega} f^{\prime \prime}(u(t))\left(v+t \phi_{1}\right) e(t) k_{2}(t),
\end{aligned}
$$

and by (7.5)

$$
\int_{\Omega}\left[\left|\nabla k_{2}\right|^{2}-\lambda k_{2}{ }^{2}\right] \geq C\left\|k_{2}\right\|_{2}^{2} \geq 0,
$$

where $C>0$. From (9), (10) and (1.10)

$$
\eta\left\|u(t) k_{2}(t)\right\|_{2}^{2} / 2 \leq(\alpha(t))^{-1}\left|\alpha^{\prime}(t)\right|\left\|f^{(3)}\right\|_{\infty}\left\|u(t) k_{2}(t)\right\|_{2}\|u(t) e(t)\|_{2}
$$

and (12) $C\left\|k_{2}(t)\right\|_{2}^{2}$ has the same upper bound. Restate (6) as

$$
\|u(t) e(t)\|_{2} \leq K_{1}(f, \Omega)(\alpha(t))^{2}\left|\alpha^{\prime}(t)\right|^{-1},
$$

use (13) in (11) to conclude that

$$
\left\|u(t) k_{2}(t)\right\|_{2} \leq K_{4} \alpha(t),
$$

and use (13) and (14) in (12) to conclude that (15) $\left\|(\alpha(t))^{-1} k_{2}\right\|_{2}$ is bounded above. The desired conclusion (3) (and hence (1)) results from (8) and (15).

To prove (2), let $\varepsilon>0$. We will show that (16) $|C|<\varepsilon$ on $\Lambda$. Choose $\xi>0$ such that, if $|s|<\xi$, then

$$
\left|f^{(3)}(s)-f^{(3)}(0)\right|<\varepsilon / 4 K_{2}(f, \Omega) ;
$$

here $K_{2}(f, \Omega)=\lambda_{2}^{2 i}(K(\Omega))^{2}(m(\Omega))^{1 / 2} K_{1}(f, \Omega)$, where the constants are given by (5.1) and (13) (i.e. (6)) and $m(\Omega)$ is the measure of $\Omega$. Let $\Omega_{1}=\{x \in \Omega$ : $|u(t)(x)|<\xi\}$, let $\Omega_{2}=\Omega-\Omega_{1}$, and let

$$
K_{3}(f, \Omega)=2\left\|f^{(3)}\right\|_{\infty} \lambda_{2}{ }^{2 i}(K(\Omega))^{2} K_{1}(f, \Omega) .
$$


Let $\delta>0, s>0$ and $T \subset \Omega$ be as given by (6.1)(vi) for $\bar{u}=e(t)$ and $\varepsilon_{1}=\left(\varepsilon / 3 K_{3}(f, \Omega)\right)^{2}$; thus $e(t) \geq s$ on $T$ and $m(\Omega-T)<\varepsilon_{1}$. Let $S=$ $\{x \in T:|u(t)(x)| \geq \xi\}$. From (1.10) $\eta(u(t))^{2} / 2 \leq f^{\prime}(u(t))$ and from (5.2)(2) $\|u(t) e(t)\|_{2} \rightarrow 0$ as $\delta \rightarrow 0$; thus we may further choose $\delta$ so that

$$
\|u(t) e(t)\|_{2}<\xi_{S \varepsilon_{1}}{ }^{1 / 2}
$$

Since $\|u(t) e(t)\|_{2} \geq \xi s(m(S))^{1 / 2}, m(S)<\varepsilon_{1}$, and since $\Omega_{2} \subset(\Omega-T) \cup S$,

$$
m\left(\Omega_{2}\right)<2 \varepsilon_{1}<\left(\varepsilon / 2 K_{3}(f, \Omega)\right)^{2} .
$$

Let $\Omega_{0}=\Omega$ and let

$$
C_{i}=\alpha^{\prime}(t)(\alpha(t))^{-2} \int_{\Omega_{i}}\left[u(t) f^{(3)}(u(t))-f^{\prime \prime}(u(t))\right](e(t))^{3} \quad(i=0,1,2) ;
$$

since $F=0$ on $\Lambda$,

$$
C=C_{0}=C_{1}+C_{2} \text { on } \Lambda \text {. }
$$

By (1.10) and Taylor's formula, for each real $s \neq 0$ there exists $\zeta$ between 0 and $s$ with

$$
s f^{(3)}(s)-f^{\prime \prime}(s)=s\left[\left(f^{(3)}(s)-f^{(3)}(0)\right)+\left(f^{(3)}(0)-f^{(3)}(\zeta)\right)\right],
$$

so that by 17 ),

$$
\begin{aligned}
\left|C_{1}\right| & <\left(\varepsilon / 2 K_{2}(f, \Omega)\right)\left|\alpha^{\prime}(t)\right|(\alpha(t))^{-2} \int_{\Omega_{1}}|u(t) e(t)|(e(t))^{2} \\
& \leq\left(\varepsilon / 2 K_{1}(f, \Omega)\right)\left|\alpha^{\prime}(t)\right|(\alpha(t))^{-2}\|u(t) e(t)\|_{2} \leq \varepsilon / 2,
\end{aligned}
$$

by (5.1), (6), (13) and the Cauchy-Schwarz inequality.

By (1.10) and (20)

$$
C_{2}=\alpha^{\prime}(t)(\alpha(t))^{-2} \int_{\Omega_{2}}\left(f^{(3)}(u(t))-f^{(3)}(\zeta(t))\right)(u(t) e(t))(e(t))^{2},
$$

where $\zeta(t)(x)$ is between 0 and $u(t)(x)$ for $x \in \Omega_{2}$. As above, since $u(t)=$ $\alpha(t)\left(v+t \phi_{1}\right)$,

$$
\begin{aligned}
\left|C_{2}\right| & \leq 2\left\|f^{(3)}\right\|_{\infty} \lambda_{2}^{2 i}(K(\Omega))^{2}(m(\Omega))^{1 / 2}\left|\alpha^{\prime}(t)\right|(\alpha(t))^{-2}\|u(t) e(t)\|_{2} \\
& \leq K_{3}(f, \Omega)(m(\Omega))^{1 / 2}<\varepsilon / 2,
\end{aligned}
$$

by (13), (6) and (18). From (19), (21) and (22), the desired conclusion (16) (and thus 2) results.

In case $f(u)=u^{3}$, a simplified proof with $F\left(v+t \phi_{1}\right)=\int_{\Omega}\left(v+t \phi_{1}\right)(e(t))^{3}$ shows that, for some $a>0, \partial F / \partial t \geq a$ on all of $\Gamma$.

7.8. Lemma. Assume $\left\|f^{3}\right\|_{\infty}<\infty$. If $T=\left\{u \in H \cap L^{\infty}(\Omega): u\right.$ is real analytic $\}$ (7.6)(6), $\delta>0$ is sufficiently small and $\lambda_{1}<\lambda<\lambda_{1}+\delta$, then $T \cap \Lambda$ (7.6)(2) is contractible.

Proof. Fix $v \in\left(T \cap H_{1}\right)-\{0\}$ (7.6). For each $t \in \mathbb{R}, v+t \phi_{1} \in T-L \subset \Gamma$ (7.6) (2), (6), so that $F\left(v+t \phi_{1}\right)(7.6)(5)$ may be defined. By $(1.10) f^{\prime \prime}(s)=s g(s)$, where $g: \mathbb{R} \rightarrow \mathbb{R}$ is continuous with $0<\eta \leq g(s) \leq\left\|f^{(3)}\right\|_{\infty}$.

From (5.4), (5.1) and [A, p. 97]

$$
\int_{\Omega} g(u(t)) \phi_{1}(e(t))^{3}>\left(\eta \int_{\Omega} \phi_{1}{ }^{4}\right) / 2>0
$$


for $\delta$ sufficiently small, and from $(5.1)\left|\int_{\Omega} g(u(t)) v(e(t))^{3}\right|$ is bounded. By the positivity of $\phi_{1}$ [GT, Theorem 8.38, p. 214] and $e(t)$ [III, (1.6), p. 202], for $|t|$ large

$$
t^{-1}(\alpha(t))^{-1} F\left(v+t \phi_{1}\right)=t^{-1} \int_{\Omega} g(u(t)) v(e(t))^{3}+\int_{\Omega} g(u(t)) \phi_{1}(e(t))^{3}>0
$$

and so $F\left(v+t \phi_{1}\right)$ has the same sign as $t$. Since $\partial F / \partial t>0$ on $\Lambda$ (where $F=0)$ (7.7) for $\delta$ sufficiently small, there is a unique $t=t(v) \in \mathbb{R}$ such that $F\left(v+t(v) \phi_{1}\right)=0$ and it is a continuous (real analytic if $f(u)=u^{3}$ ) function of $(\mathrm{v})([\mathrm{Z}, \mathrm{pp} .150-151]$ and $(7.6)(5))$.

Thus $\Upsilon:\left(T \cap H_{1}\right)-\{0\} \rightarrow T \cap \Lambda$ defined by $\Upsilon(v)=v+t(v) \phi_{1}$ is a homeomorphism (real analytic diffeomorphism if $\left.f(u)=u^{3}\right)$, while $\left(T \cap H_{1}\right)-$ $\{0\}$ is homeomorphic to $T \cap H_{1}$ [Kl], so $T \cap \Lambda$ is contractible.

7.9. Proposition. Sobolev case. For $\delta>0$ sufficiently small and $\lambda_{1}<\lambda<$ $\lambda_{1}+\delta,\left(S A_{\lambda}, C A_{\lambda}\right)$ is homeomorphic $\left(C^{\infty}\right.$ diffeomorphic if $f$ is $\left.C^{\infty}\right)$ to $\left(H, H_{1}\right)$, where $H_{1}$ is a codimension one subspace of $H$.

Proof. We will prove that 1) $\pi_{1}(\Lambda, w)$ is trivial for some $w \in \Lambda$. It follows from (7.6) (3) that $\pi_{1}\left(C A_{\lambda}, u\right)$ is trivial for some $u \in C A_{\lambda}$ and from (7.4) (iii) that $C A_{\lambda}$ is $Z$-acyclic, and thus [CT-2, (2.1)] is contractible. The argument of the first paragraph of (3.9) yields the conclusion.

Let $\delta>0$ be given by (7.8), and let $\lambda_{1}<\lambda<\lambda_{1}+\delta$. Choose $w \in T \cap \Lambda \neq \varnothing$ (7.8), and let $[\alpha] \in \pi_{1}(\Lambda, w)$. There are a $C^{\infty}$ embedding $\beta:\left(S^{1}, 1\right) \rightarrow$ $(\Lambda, w)$ representing $[\alpha]$, and $\varepsilon>0$ such that $(2)$ the $\varepsilon$-neighborhood $N_{\varepsilon}$ of $\beta\left(S^{1}\right)$ in $H$ is contained in $\Gamma(7.6)$ (2). Since $\beta\left(S^{1}\right)$ has a tubular neighborhood in $\Lambda$ [L, Theorem 9, p. 96 and pp. 32-34], we may suppose that (3) for all $\gamma:\left(S^{1}, 1\right) \rightarrow(\Lambda, w)$, with $\|\gamma(s)-\beta(s)\|_{H}<\varepsilon$ for all $s \in S^{1}, \gamma$ also represents $[\alpha]$. By (7.8) it suffices (4) to find $\gamma$ satisfying (2) with $\gamma\left(S^{1}\right) \subset T \cap \Lambda$.

For each loop $\eta:\left(S^{1}, 1\right) \rightarrow(H, \eta(1))$ and $q \in \mathbb{R}$, define $\eta_{q}:\left(S^{1}, 1\right) \rightarrow$ $\left(H, \eta_{q}(1)\right)$ by $\eta_{q}(s)=\eta(s)+q \phi_{1}$ for each $s \in S^{1}$; for $|q| \lambda_{1}<\varepsilon, \beta_{q}\left(S^{1}\right) \subset \Gamma$. Pick $r>0$ and $\lambda>0$ such that $r \lambda_{1}+\lambda<\varepsilon$. Thus (5) if $\eta:\left(S^{1}, 1\right) \rightarrow$ $(H, \eta(1))$ with $\|\eta(s)-\beta(s)\|_{H}<\lambda$, then $\eta_{q}\left(S^{1}\right) \subset N_{\varepsilon} \subset \Gamma$ for $|q| \leq r$. Since $\beta\left(S^{1}\right) \subset \Lambda, F$ is 0 on $\beta\left(S^{1}\right)(7.6)(5)$, and by (7.7) for each $v+t \phi_{1} \in \beta\left(S^{1}\right)$, there exists $r=r\left(v+t \phi_{1}\right)>0$ with $r \lambda_{1}+\lambda<\varepsilon$ and $F\left(v+(t+s) \phi_{1}\right)>0$ [resp., $<0$ ] for $0<s \leq r$ [resp., $-r \leq s<0$ ]. By the continuity of $F$ and the positivity of $\partial F / \partial t$ on $\Lambda$ (7.7), this $r$ will work for nearby points on $\beta\left(S^{1}\right)$, and by the compactness of $\beta\left(S^{1}\right)$ one $r>0$ will suffice for all of $\beta\left(S^{1}\right)$. Thus there exists $b>0$ such that $F \geq b$ [resp., $\leq-b$ ] on $\beta_{r}\left(S^{1}\right)$ [resp., $\beta_{-r}\left(S^{1}\right)$ ]. (6) We may choose $\lambda$ sufficiently small that $F>b / 2$ [resp., $<-b / 2$ ] on $\eta_{r}\left(S^{1}\right)$ [resp., $n_{-r}\left(S^{1}\right)$ ] for $\eta$ satisfying (5).

Using the convexity of $T$ and the denseness of $T-L$ in $\Gamma$ (Sobolev case) (7.6)(6), we may find a polygonal embedded loop $\eta:\left(S^{1}, 1\right) \rightarrow(T-L, w)$ satisfying (5) and (6) with each line segment $X$ of $\eta\left(S^{1}\right)$ not parallel to $\phi_{1}$. For each $u=v+t \phi_{1} \in X$ there exists a unique $q(u),-r<q(u)<r$, such that $F\left(u+q(u) \phi_{1}\right)=0($ since $\partial F / \partial t>0(7.7)), q$ is $C^{k-1}$ (real analytic if $f(u)=$ $\left.u^{3}\right)$ on $X\left[\mathrm{Z}\right.$, pp. 150-151] and $q(w)=0$. Define $\gamma(s)=\eta(s)+q(\eta(s)) \phi_{1}$ for $s \in S^{1}$. Then $\gamma\left(S^{1}\right) \subset T \cap \Lambda(7.6)(5)$ and (6), $\gamma(1)=w$ and $\gamma$ satisfies (3); by (4) the conclusion results. 
7.10. Lemma. Hölder case with $\left\|f^{(3)}\right\|_{\infty}<\infty$. Let $H^{\prime}\left[\right.$ resp., $\left.H^{\prime \prime}\right]$ be $C_{0}^{2, \alpha}(\Omega)$ with the $L^{\infty}(\Omega)\left[\right.$ resp., $\left.L^{2}(\Omega)\right]$ norm.

(i) The functions $\lambda_{1}: H^{\prime} \rightarrow \mathbb{R}$ and $e: H^{\prime} \rightarrow H^{\prime \prime}$ [III, (1.9)] are continuous, and

(ii) $\Gamma$ (7.6) is open in $H^{\prime}$.

(iii) In (7.6) $\alpha: \Gamma \cap H^{\prime} \rightarrow \mathbb{R}$, $\tilde{e}: \Gamma \cap H^{\prime} \rightarrow H^{\prime \prime}$ defined by $\tilde{e}(u)=e(\alpha(u) u)$, and $F: \Gamma \cap H^{\prime} \rightarrow \mathbb{R}$ are continuous.

Proof. The (1) continuity of $\lambda_{1}(u)$ is immediate from its infimum definition [III, (1.1)]. Let $L$ and $N$ be the operators of $W_{0}^{1,2}(\Omega)=W$ into itself defined in [II, (1.4), p. 228], let $\lambda>0$, let $u \in H=C_{0}^{2, \alpha}(\Omega)$ and define $T=T_{\lambda, u}=$ $L-(1 / \lambda) D N(u)$. Then

$$
\left\langle T_{\lambda, u} \phi, \psi\right\rangle_{W}=\int_{\Omega} \phi \psi-\left(\int_{\Omega} f^{\prime}(u) \phi \psi\right) / \lambda
$$

for all $\phi$ and $\psi$ in $W_{0}^{1,2}(\Omega)$ and $T$ is a compact selfadjoint linear operator. Since (2) $\|\phi\|_{W}$ is the sup of $\langle\phi, \psi\rangle_{W}$ over $\psi \in W$ with $\|\psi\|_{W}=1$, it follows from (1) that (3) the map $u \rightarrow T_{\lambda_{1}(u), u}$ is continuous, where the domain is $H^{\prime}$ and the range has the operator norm induced from $W$. From [Ka1, Theorem 2.13 , p. 203 and pp. 212-214] (4) the map of $T$ into its first eigenvector is continuous, and conclusion (i) results from (3), (4) and [A, p. 97].

Let $\bar{u} \in \Gamma \cap H^{\prime}$, so that $\lambda_{1}(\alpha(\bar{u}) \bar{u})=\lambda$ and $\alpha(\bar{u}) \bar{u} \in S A_{\lambda}$, and let $\varepsilon>0$. We may suppose that $\varepsilon<\alpha(\bar{u})$, and let $c_{i}=\alpha(\bar{u})+(-1)^{i} \varepsilon(i=1,2)$. Since $\lambda_{1}(c \bar{u})$ is increasing in $c$ (3.6), by (1) there exists $\delta>0$ such that, if $\|u-\bar{u}\|_{\infty}<\delta$ then $\lambda_{1}\left(c_{1} u\right)<\lambda=\lambda_{1}(\alpha(\bar{u}) \bar{u})<\lambda_{1}\left(c_{2} u\right)$; thus there is a unique $\alpha(u) \in \mathbb{R}, c_{1}<$ $\alpha(u)<c_{2}$, such that $\lambda_{1}(\alpha(u) u)=\lambda$, so that $u \in \Gamma$ and $|\alpha(u)-\alpha(\bar{u})|<\varepsilon$. Conclusion (ii) results, and (5) $\alpha: \Gamma \cap H^{\prime} \rightarrow \mathbb{R}$ is continuous.

From (5) and (i), $\tilde{e}$ is continuous; and by standard inequalities using (2), $F: \Gamma \cap H^{\prime} \rightarrow \mathbb{R}$ is continuous.

7.11. Proposition. In the Hölder case with $\left\|f^{(3)}\right\|_{\infty}<\infty$, (7.9) holds (with homeomorphism even for $f C^{\infty}$ ).

Proof. We modify the argument of (7.9). In the first paragraph, since $C_{0}^{2, \alpha}(\bar{\Omega})$ is not a separable Hilbert space, the use of [BH, Theorem 0.1, p. 1261] in (3.9) yields only a homeomorphism.

The set $\Lambda$ (7.6) is defined by $F\left(v+t \phi_{1}\right)=0$; let $\bar{v}+\bar{t} \phi_{1} \in \Lambda$ and let $\zeta>0$. We may suppose that $\zeta$ is sufficiently small that $\bar{v}+t \phi_{1} \in \Gamma$ for $|t-\bar{t}| \leq \zeta$ (7.10)(ii). Since $\partial F / \partial t>0$ on $\Lambda$ (7.7), $F\left(\bar{v}+\zeta \phi_{1}\right)>0$ and $F\left(\bar{v}-\zeta \phi_{1}\right)<0$; thus for some $\xi>0$, if $\|v-\bar{v}\|_{\infty}<\xi$, then $F\left(v+\zeta \phi_{1}\right)>0$ and $F\left(v-\zeta \phi_{1}\right)<0$ (7.10)(iii). From (7.7) there is a unique $t(v), \bar{t}-\zeta<t(v)<\bar{t}+\zeta$, such that $F\left(v+t(v) \cdot \phi_{1}\right)=0$; hence the germ $t(v)$ given by the implicit function theorem [Z, pp. $150-151]$ on $H=C_{0}^{2, \alpha}(\bar{\Omega})$ is continuous in $v$ in $\Lambda \cap H^{\prime}$ (7.10) (as well as $C^{k-1}$ (real analytic if $\left.f(u)=u^{3}\right)$ in $\left.\Lambda \cap H\right)$.

By a $C^{0}$ partition of unity argument, there are continuous functions $g_{i}$ : $\Lambda \cap H^{\prime} \rightarrow \mathbb{R}(i=1,2)$ such that, for each $\bar{v}+\bar{t} \phi_{1} \in \Lambda$ and $t$ satisfying $g_{1}\left(\bar{v}+\bar{t} \phi_{1}\right)<t<g_{2}\left(\bar{v}+\bar{t} \phi_{1}\right), \bar{v}+t \phi_{1} \in \Gamma$. The set of such $\bar{v}+t \phi_{1}$ constitute 
a subset $U$ open in $\Gamma \cap H^{\prime}$, and (1) $\Lambda \cap H^{\prime}$ is a deformation retract of $U$ with retraction homotopy $\psi: U \times[0,1] \rightarrow U, \psi_{h}(u)=\psi(u, h)$ and $h \in[0,1]$.

Let $\delta>0$ be given by (7.8), and let $\lambda_{1}<\lambda<\lambda_{1}+\delta$. Choose $w \in T \cap \Lambda \neq \varnothing$ (7.8), and let $\beta:\left(S^{1}, 1\right) \rightarrow(\Lambda, w)$ be a map. (2) There exists $\mu>0$ such that, if $s \in S^{1}, u \in H^{\prime}$ and $\|u-\beta(s)\|_{\infty}<\mu$, then $u \in \Gamma$ (7.10)(ii). By (1) there exists $\varepsilon>0, \varepsilon<\mu$, such that if $\gamma:\left(S^{1}, 1\right) \rightarrow(\Gamma, w)$ with $(*)$ $\|\beta(s)-\gamma(s)\|_{\infty}<\varepsilon$ for all $s \in S^{1}$, then $\left\|\beta(s)-\psi_{h}(\gamma(s))\right\|_{\infty}<\mu$ for $h \in[0,1]$; and the homotopy $(1-h) \beta(s)+h \gamma(s)$ satisfies condition $(*)$. Thus (by (2)), (3) if $\gamma:\left(S^{1}, 1\right) \rightarrow(\Lambda, w)$ with $\|\beta(s)-\gamma(s)\|_{\infty}<\varepsilon$ for all $s \in S^{1}$, then the homotopy classes (in $\Lambda$ ) $[\beta]=[\gamma]$. By (7.8) it suffices (4) to find $\gamma$ satisfying (3) with $\gamma\left(S^{1}\right) \subset T \cap \Lambda$.

We now follow the last two paragraphs of the proof of (7.9), replacing $H$ by $H^{\prime}$ (7.10), $|q| \lambda_{1}$ by $|q|\left\|\phi_{1}\right\|_{\infty}, r \lambda_{1}+\lambda$ by $r\left\|\phi_{1}\right\|_{\infty}+\lambda$, and $\|\eta(s)-\beta(s)\|_{H}$ by $\|\eta(s)-\beta(s)\|_{\infty}$, and using (7.10).

\section{The MAP $A$ IS THE GLOBAL CUSP MAP}

Here we prove the main theorem (1.1) as (8.3) and (8.5). The notions of topological $=C^{0}$ singular set $S \Phi$, fold point, and cusp point, used in (8.1) and (8.2), are defined in (1.2) and (1.5). The set of cusp points of $A$ is denoted by $C A$.

The symbol $\approx$ below refers to homeomorphism, and $Z$ is the ring of integers.

\subsection{Proposition [CT-2, (2.1)]. Suppose}

(i) $H$ and $H^{\prime}$ are Fréchet spaces with dimension at least two;

(ii) $\Phi: H \rightarrow H^{\prime}$ is a proper continuous function;

(iii) at any point of $S \Phi, \Phi$ is a (topological) fold or cusp;

(iv) the restriction map $\Phi \mid S \Phi$ is injective;

(v) $(H, S \Phi) \approx(H, \Gamma)$, where $\Gamma$ is a codimension one subspace of $H$; and

(vi) $(S \Phi, C \Phi) \approx(\Gamma, \Lambda)$, where $\Lambda$ is a codimension one subspace of $\Gamma$.

Then $\Phi$ is $C^{0}$ equivalent (1.4) to the global cusp map.

\subsection{Corollary [CT-2, (2.2)]. Suppose}

(i) $H$ and $H^{\prime}$ are Fréchet spaces, each with dimension infinite or two;

(ii) (iii) and (iv) as in (8.1);

(v) $S \Phi$ is simply connected and Z-acyclic;

(vi) each component of $C \Phi$ is simply connected; and

(vii) there exists $y \in H^{\prime}-\Phi(S \Phi)$ such that $\Phi^{-1}(y)$ is a single point.

Then $\Phi$ is $C^{0}$ equivalent to the global cusp map.

We use either (8.1) or (8.2) in (8.3) and (8.5).

8.3. Theorem. Let $A$ be a $C^{k}(k=3,4, \ldots$, or $\infty)$ map satisfying (1.10) with $\left\|f^{(3)}\right\|_{\infty}<\infty$. If $\varepsilon>0$ is sufficiently small, then

$$
A: H \times\left(-\infty, \lambda_{1}+\varepsilon\right) \rightarrow H \times\left(-\infty, \lambda_{1}+\varepsilon\right)
$$

is $C^{0}$ (1.4) equivalent to the global cusp map.

Proof. Let $\varepsilon>0$ be sufficiently small that $S A$ has only folds and cusps (5.2) and the restriction map $A \mid S A$ is injective (6.5) for $\lambda<\lambda_{1}+\varepsilon$. It suffices to 
prove that $A$ satisfies conditions (i)-(vi) of the characterization of global cusp map (8.1). It is immediate that $H$ is a Fréchet space with dimension at least two; (ii) $A$ is proper [II, (2.8)]; (iii) $S A$ has only folds and cusps (5.2); (iv) $A \mid S A$ is injective (6.5);

$$
\left(H \times\left(-\infty, \lambda_{1}+\varepsilon\right),\left(H \times\left(-\infty, \lambda_{1}+\varepsilon\right) \cap S A\right)\right) \approx(H \times \mathbb{R}, H \times 0)
$$

since $S A$ is the graph of a $C^{k-1}$ function (real analytic if $f(u)=u^{3}$ ) [III, (1.6), (1.8) and (2.4)]; and (vi) $(S A, C A) \approx(H, \Gamma)$, where $\Gamma$ is a codimension one subspace of $H((7.4)$ and [III, (2.4)]).

8.4. Remark. Suppose that $u \in S A_{\lambda}-C A_{\lambda}$. By (8.3) there is precisely one $v \in H$ with $v \neq u$ and $A_{\lambda}(v)=A_{\lambda}(u)$, and $v \notin S A_{\lambda}$. If $u \in S_{+} A_{\lambda}$ (i.e. $J(u)>0(3.1))$ [resp., $u \in S_{-} A_{\lambda}$ ], then $u(x)-v(x)>0$ a.e. on $\Omega$ and $\beta<0$ [resp., $u(x)-v(x)<0$ a.e. on $\Omega$ and $\beta>0$ ] by (7.4)(ii) and (iii). Now

$$
\left\langle u-v, \phi_{1}\right\rangle_{H}=\int_{\Omega} \nabla(u-v) \nabla \phi_{1}=\lambda_{1} \int_{\Omega}(u-v) \phi_{1}>0
$$

[resp., < 0], [GT, p. 214, Theorem 8.38].

Now consider $\delta \rightarrow 0$, i.e. $\lambda_{1}(u)=\lambda \rightarrow \lambda_{1}$. Then $u-v=(-\beta)(e+\bar{w})$ (6.4)(iii), where $-\beta>0$ [resp., $<0$ ], $|\beta| \rightarrow 0$ (6.4)(iv)(a), $\|w\|_{H} \leq K|\beta|$ $(6.4)(\mathrm{iv})(\mathrm{c})$, and $\left\|e-\phi_{1}\right\|_{H} \rightarrow 0$ (5.5). Thus $\|u-v\|_{H} \rightarrow 0$ and $u-v$ points almost in the direction $-\phi_{1}$ (and $-e$ ) [resp., $\phi_{1}$ (and $e$ )]. Note that $D J(u)$. $e>0$ (5.2) near $C A_{\lambda}$, so that $u-v$ points from $S_{-} A_{\lambda}$ to $S_{+} A_{\lambda}$ [resp., $S_{+} A_{\lambda}$ to $S_{-} A_{\lambda}$ ] for $u-v$ near $C A_{\lambda}$ and $\lambda$ near $\lambda_{1}$.

In [CT-2, (2.6)-(2.9)] a notion of pseudo cusp map $\Phi$ is defined and its structure discussed. It is characterized by satisfying $(8.2)(\mathrm{i})-(\mathrm{v})$ and (vii), and it is a consequence of these properties that the cusp set $C \Phi$ is $Z$-acyclic [CT-2, proof of (2.1)]. Thus, $\Phi$ is $C^{0}$ equivalent to the global cusp map if and only if $C \Phi$ is simply connected.

8.5. Theorem. Assume $f(u)=u^{3}$ (and $n \leq 3$ ). Suppose $\lambda_{1}<\lambda<\lambda_{1}+\varepsilon$ for $\varepsilon>0$ sufficiently small. Then $A_{\lambda}$ is $C^{0}$ equivalent to the global cusp map.

Proof. Let $\varepsilon>0$ be sufficiently small that $A$ has only folds and cusps (5.2) for $\lambda_{1}<\lambda<\lambda_{1}+\varepsilon$ and $A \mid S A$ is injective (6.5). It suffices to prove that $A_{\lambda}$ satisfies conditions (i)-(vi) of (8.1). Now (i) $H=W_{0}^{1,2}(\Omega)$ is a separable Hilbert space [A, p. 47, (3.5)]; (ii) $A_{\lambda}: H \rightarrow H$ is a proper map (2.1); (iii) the only singular points of $A_{\lambda}$ are folds and cusps ((5.2) and (3.1)); (iv) $A_{\lambda} \mid S A_{\lambda}$ is injective, since $A \mid S A$ is injective (6.5); (v) $\left(H, S A_{\lambda}\right)$ is homeomorphic $\left(C^{\infty}\right.$ diffeomorphic in the Sobolev case) to $\left(H, H_{1}\right)\left((3.9)\right.$ and (3.13)), where $H_{1}$ is a codimension one subspace of $H$; and (vi) $\left(S A_{\lambda}, C A_{\lambda}\right)$ is homeomorphic ( $C^{\infty}$ diffeomorphic in the Sobolev case) to $\left(H_{1}, \Lambda\right)$, where $\Lambda$ is a codimension one subspace of $H_{1}((7.9)$ and (7.11)).

Alternatively, we could use (8.2) instead. It then suffices to prove (vii) for some $y \in H-A_{\lambda}\left(S A_{\lambda}\right), A_{\lambda}^{-1}(y)$ is a single point. From (v), (iii) and (1.5) $A_{\lambda}$ has a fold point $u$; by $(3.1)(u, \lambda)$ is a fold point of $A$. Since $A$ is $C^{0}$ equivalent to the standard cusp map $(8.3), A^{-1}(A(u, \lambda))$ consists of $(u, \lambda)$ and a regular point $(v, \lambda)$ of $A$, and so $A_{\lambda}^{-1}\left(A_{\lambda}(u)\right)$ consists of the fold point $u$ and the regular point $v$. From (ii) there is a connected open neighborhood $W$ of $A_{\lambda}(u)$ sufficiently small that $A_{\lambda}^{-1}(W)$ consists of two components, $W_{1}$ 
and $W_{2}$ with $W_{1} \stackrel{A}{\longrightarrow} W C^{\infty}$ equivalent to the fold map and $W_{2} \stackrel{A}{\longrightarrow} W$ a $C^{\infty}$ diffeomorphism (onto). Condition (vii) results, and the desired conclusion follows.

\section{APPENDIX. Application of [II] and [III] to $-\Delta u-\lambda u+f(u)=g$}

The results of [II] and [III], which are used extensively in this paper, are given for $-\Delta u-\lambda u+u^{3}=g$ on $W_{0}^{1,2}(\Omega)$ and for a more general mapping called Abstract A on a Hilbert space. In this paper we apply these results to $-\Delta u-\lambda u+f(u)=g$ on $W_{0}^{1,2}(\Omega)$ and on the Hölder spaces $C_{0}^{2, \alpha}(\bar{\Omega})$ and $C^{0, \alpha}(\bar{\Omega})$, and this appendix gives the hypotheses required. A citation elsewhere in this paper to [II] or [III] implicitly involves reference to (A.2) or (A.3).

A.1. Remark. (a) If $f: \mathbb{R} \rightarrow \mathbb{R}$ is $C^{2}, f^{\prime}(s) \geq 0$ and $f^{\prime \prime}(s) \neq 0$ for all $s \neq 0$, and $f^{\prime}(0)=0$, then $f^{\prime}(s)>0$ and $s f^{\prime \prime}(s)>0$ for $s \neq 0$, and $f^{\prime \prime}(0)=0$.

(b) If, in addition, $f$ is $C^{3}$ and $f^{(3)}(s) \neq 0$ for all real $s$, then $f^{(3)}(s)>0$ for all real $s$. (Use the Mean Value Theorem.)

A.2. Remark. Application of [II] and [III] to $-\Delta u-\lambda u+f(u)=g$ on $\Omega, u \mid \partial \Omega=0$, where $\Omega$ is bounded in $\mathbb{R}^{4}, n \leq 4, f: \mathbb{R} \rightarrow \mathbb{R}, f(0)=$ $0, A_{\lambda}: H \rightarrow H$, and $H$ is the Sobolev space $W_{0}^{1,2}(\Omega)$.

(i) First, we discuss the application of theorems about Abstract $A$ [II, (1.2)] (see [II, (1.3) and (1.4)]). Whenever an hypothesis in [II] or [III] includes "Abstract A", assume that $n \leq 4, f$ is $C^{3}, f^{(3)} \in L^{\infty}(\mathbb{R})$, and $f^{\prime}(s) \geq 0$ for all $s \in \mathbb{R}$. Condition $(1.2)(2)$ is always satisfied; $(1.2)(3)(b)$ is satisfied if $f^{\prime}(s)>0$ for all real $s \neq 0$ and $f^{\prime}(0)=0 ;(1.2)(3)\left(\mathrm{c}_{j}\right)$ results if $f^{(j)}(0)=0$ and $(1.2)(3)(\mathrm{c})$ is $c_{j}$ for $j=0,1$, and 2 ; and $(1.2)(3)(\mathrm{d})$ results if $f^{(3)}(s)>0$ for all real $s$. (Condition $(1.2)(3)(\mathrm{e})$ is used only in conjunction with $(1.2)(3)(\mathrm{c})$, and this requires $f(s)=a s^{3}$ for $a \in \mathbb{R}$.) For $A$ to be $C^{k}$ with $k \geq 4$, it is necessary that $f$ be $C^{k}$, but not sufficient.

(ii) Second, we consider the generalization of some of the theorems about standard $A$ [II, (1.3)], i.e. replacing $-\Delta u-\lambda u+u^{3}=g$ by $-\Delta u-\lambda u+f(u)=g$. Theorem [II, (2.9)], which gives sufficient conditions for $A$ to be proper, is already a generalization of [II, (2.8)] to $f(u)$; its hypotheses and Abstract $A$ above should be added to those of [II, (2.10), (3.8), (3.9)] to achieve properness. On the other hand, hypotheses (1.10) suffices by (2.1). In addition, for [II, (3.9)] add $(1.2)(3)(b)-(d)$ above. Propositions [III, (1.6), (1.9)(a) and $(1.10)(\mathrm{c})(\mathrm{d})$ ] generalize if $n \leq 3$, we assume the hypotheses above for Abstract $\mathrm{A}$, and, in the case of $(1.9)(a)$ assume $(1.2)(3)(c)$ and for $(1.10)(\mathrm{c})(\mathrm{d})$, assume also that $f^{\prime \prime}(s) \neq 0$ for $s \neq 0$ and $f^{\prime \prime}(0)=0$ (see (A.1)). Proposition [III, (2.9)] generalizes under the hypotheses given above for Abstract A and for (1.2)(3)(b)(d) (thus $s f^{\prime \prime}(s)>0$ for $s \neq 0$ ); in [III, (2.9)(b)] also assume $n \leq 3$.

Propositions [III, (1.11) and (1.12)] generalize if $f$ is $C^{k}$ where $k>n+$ $2, f$ satisfies the hypotheses given above for Abstract A and for $(1.2)(\mathrm{b})(\mathrm{c})$ and $f^{\prime \prime}(s) \neq 0$ for all real $s \neq 0$. (For the proof replace (1) by $\varphi(v, \lambda, u)=\Delta v-$ $f^{\prime}(u) v+\lambda v=0$ and $D_{2} \varphi(v, \lambda, u) \cdot(0,0, z)=-f^{\prime \prime}(u) v z,(2)$ by $\int_{\Omega} f^{\prime \prime}(u) v z w$ $=0$ and (4) by $f^{\prime \prime}(u) v \equiv 0$ on $\Omega$.) 
(iii) In definition [II, (3.1)] we have

$$
\begin{aligned}
& \text { (fold 2) } \int_{\Omega} f^{\prime \prime}(u) e^{3} \neq 0, \\
& (\operatorname{cusp} 2) \int_{\Omega} f^{\prime \prime}(u) e^{3}=0 \\
& (\operatorname{cusp} \tilde{4}) \int_{\Omega} f^{(3)}(u) e^{4}-3 \int_{\Omega} f^{\prime \prime}(u) e^{2} y \neq 0,
\end{aligned}
$$

where $y=\left(D A_{\lambda}(u)\right)^{-1}\left(D^{2} A_{\lambda}(u)(e, e)\right)$. Cusp 3 is always satisfied for $A$ at any $(u, \lambda) \in H \times R$ with $\omega=(0,1)$ [II, (3.5), p. 237], and for $A_{\lambda}$ at any $u \neq 0$ with $\omega=u$ (by the first two paragraphs of the proof of [CT-1, (1.8), p. 63]).

A.3. Remark. Application of [II] and [III] to $A_{\lambda}(u)=-\Delta u-\lambda u+f(u)=g$ on $\Omega, u \mid \partial \Omega=0$, where $\Omega$ is $C^{2, \alpha}$ bounded in $\mathbb{R}^{n}$ ( $n$ is arbitrary), $f: \mathbb{R} \rightarrow \mathbb{R}$ is $C^{2}, f(0)=0$, and $A_{\lambda}: C_{0}^{2, \alpha}(\bar{\Omega}) \rightarrow C^{\alpha}(\bar{\Omega})$ (Hölder spaces).

(i) General conditions. For Abstract $\mathrm{A}$ in this case $L$ and $N$ are maps of $C_{0}^{2, \alpha}(\bar{\Omega})$ into $C^{\alpha}(\bar{\Omega})$ defined by $L u=u$ and $N(u)=f(u)$. The conditions given in (A.2) for the Sobolev space case apply to almost all local theorems with the following changes. (Those results, mainly global, which are exceptions are listed in the next paragraph.) If Abstract $\mathrm{A}$ is required to be $C^{k}$ in a theorem of [II] or [III], or if the derivative $f^{(k)}$ is mentioned in a condition in (A.2), assume that $f$ is $C^{k+1}$. Delete all restrictions on $n$, and for Abstract A (and theorems about standard $A$ which we are generalizing here) delete the restrictions that $f$ is $C^{3}$ and $f^{(3)} \in L^{\infty}(\mathbb{R})$. In definitions (and in most proofs) interpret inner products in $H=W_{0}^{1,2}(\bar{\Omega})$ as $L^{2}(\Omega)$ inner products in $C_{0}^{2, \alpha}(\bar{\Omega})$ (for the domain of $A_{\lambda}$ ) and $C^{\alpha}(\bar{\Omega})$ (for the range of $A_{\lambda}$ ). (In [II, (1.4)] delete the hypothesis that $f^{(3)} \in L^{\infty}(\mathbb{R})$.)

(ii) Global exceptions. The main exceptions to the previous paragraph, because they require additional hypotheses, are the global results (1)-(8) listed below. All involve the hypothesis or conclusion " $A$ is a proper map" or the conclusion "homeomorphism" or "compact".

(1) [II, (1.5) and (1.6)] are replaced by $(2.2)(\mathrm{a})$.

(2) [II, (2.2)] is proved only in case $j=0$ or 1 , is $(2.4)(\mathrm{iv})$, and thus requires stronger hypotheses.

(3) [II, (2.3)] is (replaced by) (2.5)(iii).

(4) [II, (2.4] is a consequence of [II, (2.2)] and thus uses the stronger hypotheses in (2.4)(iv). Except for [II, (2.7)(ii)] all uses of [II, (2.2) and (2.4)] in proofs in [II] and [III] can be replaced by use of $(2.5)(\mathrm{i})$, which requires only the standard hypotheses that $f$ is $C^{2}, f(0)=0$, and $\Omega$ is $C^{2, \alpha}$.

(5) [II, (2.7)(ii)] requires the hypotheses of $(2.4)(\mathrm{iv})$, which replaces [II, (2.2)].

(6) [II, (2.8) and (2.9)] are (replaced by) (2.5)(ii).

(7) $[$ II, (2.10)] requires the hypotheses of $(2.5)$ (iii).

(8) [II, (3.8), (3.9), (3.10)] require the hypotheses of $(2.4)$ (iv).

(iii) Other exceptions. In addition, omit (in the Hölder case) the following results about standard $A\left(f(u)=u^{3}\right)$ : [III, (1.11)] (in its proof $C_{0}^{\infty}(\Omega)$ is not dense in $C_{0}^{2, \alpha}(\bar{\Omega})$ ) and [III, (1.12)]. In [III, (1.6)] "a.e." can be omitted. Theorem [III, (1.5)] is discussed in $(\mathrm{v})$ below. 
(iv) Eigenvalues and eigenvectors. The eigenvalue equation [III, (1.2)] $v-\lambda L v$ $+D N(u) \cdot v=0$ becomes $-\Delta v-\lambda v+f^{\prime}(u) \cdot v=0$, and the definition [III, (1.1)a] of the eigenvalues $\lambda_{j}(u)$ becomes

$$
\lambda_{j}(u)=\sup _{F \in \mathscr{S}_{j-1}} \inf _{\phi \in F,\|\phi\|_{2}=1} D_{u}[\phi],
$$

where

$$
D_{u}[\phi]=\int_{\Omega}\left[|\nabla \phi|^{2}+f^{\prime}(u) \phi^{2}\right]
$$

and $\mathscr{F}_{j-1}$ is the collection of subspaces of $C_{0}^{2, \alpha}(\bar{\Omega})$ of codimension $j-1$. For [III, (1.1)(b)] the hypotheses in (A.2) imply that $f(u)=a u^{3}$, for some real $a$, so that

$$
D_{c, u}[\phi]=\int_{\Omega}|\nabla \phi|^{2}+6 a c \int_{\Omega} u^{2} \phi^{2} .
$$

Under hypotheses (1.10) $a>0$. Since $f^{\prime}(u)$ is in $C^{1}(\bar{\Omega})$ [GT, p. 10], for $u \in C_{0}^{2, \alpha}(\bar{\Omega})$ the Sobolev eigenvectors and eigenvalues are the same as the Hölder eigenvectors and eigenvalues (see (A.4)). Thus the following results in the Sobolev case yield the corresponding results in the Hölder case: [III, (1.2), $(1.3),(1.5)(\mathrm{a}),(1.6),(2.1)-(2.8)$, and (3.1)]. (In the proof of [III, (2.6)] pick any $u \not \equiv 0$ in $C_{0}^{\infty}(\Omega)$ with support $u \subset \bar{U}$. Then $c u \in C_{0}^{2, \alpha}(\bar{\Omega})$ for all $c \in \mathbb{R}$, and by the eigenvalue comment above, the intersection of this line with the singular set $S A_{\lambda}$ is the same in both the Sobolev and Hölder cases.)

(v) [III, (1.5)]. In the statement of [III, (1.5)] replace \|\|$_{H}$ by \|\|$_{2, \alpha}$, interpret $\left\|D^{3} N(0)\right\|$ as its norm as a map of $C^{2, \alpha}(\bar{\Omega})$ into $C^{\alpha}(\bar{\Omega})$ (subscripts 0 omitted!), in (c) delete $\lambda_{j}(u)$ on the right side and make the resulting change in (d), and replace the last sentence by: For standard $A,\left\|D^{3} N(0)\right\|=6$. (In the proof of (b) use $\phi \equiv 1$ and [GT, (4.7), p. 53] to note that

$$
\left.\sup _{x \in \Omega}\left|f^{\prime}(u)-f^{\prime}(\bar{u})\right| \leq\|D N(u)-D N(\bar{u})\| .\right)
$$

Make the following changes in the proof of $(b)$ :

$$
\begin{aligned}
& \left\langle[D N(u)-D N(\bar{u})] \phi_{F, \bar{u}}, \phi_{F, \bar{u}}\right\rangle_{2}=\int_{\Omega}\left[f^{\prime}(u)-f^{\prime}(\bar{u})\right]\left(\phi_{F, \bar{u}}\right)^{2} \\
& \quad \leq\left(\sup _{x \in \Omega}\left|f^{\prime}(u(x))-f^{\prime}(\bar{u}(x))\right|\left\|\phi_{F, \bar{u}}\right\|_{2}^{2} \leq\|D N(u)-D N(\bar{u})\|\left\|\phi_{F, \bar{u}}\right\|_{2}^{2}\right) .
\end{aligned}
$$

A.4. Remark. If $\Omega \subset \mathbb{R}^{n}$ is connected open bounded with $\partial \Omega C^{2, \alpha}, u \in$ $C_{0}^{2, \alpha}(\bar{\Omega}), f: \mathbb{R} \rightarrow \mathbb{R}$ is $C^{2}$ with $f(0)=0$, and $0 \neq e \in W_{0}^{1,2}(\Omega)$ with $e \in \operatorname{ker} D A_{\lambda}(u)$, then $e \in C_{0}^{2, \alpha}(\bar{\Omega})$.

Thus, under these hypotheses, the Sobolev eigenvectors and eigenvalues are the same as those of Hölder.

Proof. By (5.1) $e \in L^{\infty}(\Omega)$. Since $-\Delta e-\lambda e+f^{\prime}(u) e=0,-\Delta e \in L^{\infty}(\Omega)$. By [LU, Theorem 15.1, p. 203] $e \in W^{2, p}(\Omega)$ for every $p$ and by [A, pp. 9798] $e \in C^{1}(\bar{\Omega})$, so that $-\Delta e \in C^{1}(\bar{\Omega}) \subset C^{0, \alpha}(\bar{\Omega})$. Thus $e \in C_{0}^{2, \alpha}(\bar{\Omega})$ [GT, Theorem 6.14, p. 107]. 


\section{REFERENCES}

[A] R. A. Adams, Sobolev spaces, Academic Press, New York, 1975.

[AP] A. Ambrosetti and G. Prodi, On the inversion of some differentiable maps with singularities, Ann. Mat. Pura Appl. (4) 93 (1972), 231-246.

[B-1] M. S. Berger, Mathematical structures of nonlinear science, Kluwer Academic Publishers, Hingham, Mass., 1990.

[B] - Nonlinearity and functional analysis, Academic Press, New York, 1977.

[BC-1] M. S. Berger and P. T. Church, Complete integrability and perturbation of a nonlinear Dirichlet problem. I, Indiana Univ. Math. J. 28 (1979), 935-952. Erratum, ibid., 30 (1981), 799.

[BC-2] _ Complete integrability and perturbation of a nonlinear Dirichlet problem. II, Indiana Univ. Math. J. 29 (1980), 715-735.

[I] M. S. Berger, P. T. Church and J. G. Timourian, Folds and cusps in Banach spaces, with applications to nonlinear partial differential equations. I, Indiana Univ. Math. J. 34 (1985), $1-19$.

[II] _ Folds and cusps in Banach spaces, with applications to nonlinear partial differential equations. II, Trans. Amer. Math. Soc. 307 (1988), 225-244.

[BP] M. S. Berger and E. Podalak, On the solutions of a nonlinear Dirichlet problem, Indiana Univ. Math. J. 24 (1975), 837-846.

[BJS] L. Bers, F. John and M. Schechter, Partial differential equations, Wiley, New York, 1964.

[BH] D. Burgehlea and D. Henderson, Smoothings and homeomorphisms for Hilbert manifolds, Bull. Amer. Math. Soc. 76 (1970), 1261-1265.

[Ca] V. Cafagna, Global invertibility and finite solvability, Nonlinear Functional Analysis (Newark, N. J., 1987), Lecture Notes in Pure and Appl. Math., vol. 121, Dekker, New York, 1990, pp. 1-30.

[CD-1] V. Cafagna and F. Donati, Singularity theory and the number of solutions to some nonlinear problems, preprint.

[CD-2] __, Un résult global de multiplicité pour un problème differentiel non linéaire du premier ordre, C.R. Acad. Sci. Paris Sér. I. Math. 300 (1985), 523-526.

[CaT] V. Cafagna and G. Tarantello, Multiple solutions for some semilinear elliptic equations, Math. Ann. 276 (1987), 643-656.

[CJM] Y. S. Choi, K. C. Jen and P. J. McKenna, The structure of the solution set for the periodic oscillations in a suspension bridge model, preprint.

[CT-1] P. T. Church and J. G. Timourian, A nonlinear elliptic operator and its singular values, Pacific J. Math. 136 (1989), 57-70.

[CT-2] __, Global cusp maps in differential and integral equations, Nonlinear Anal. (to appear).

[CT-3] _ Global fold maps in differential and integral equations, Nonlinear Anal. 181 (1992), 743-758.

[III] _ The singular set of a nonlinear elliptic operator, Michigan Math. J. 35 (1988), 197213.

[Cr] J. Cronin, Fixed points and topological degree in nonlinear analysis, Amer. Math. Soc., Providence, R. I., 1964.

[D] J. Damon, Time dependent nonlinear oscillations with many periodic solutions, SIAM J. Math. Anal. 18 (1987), 1294-1316.

[De] K. Deimling, Nonlinear functional analysis, Springer-Verlag, New York, 1985.

[Di] J. Dieudonné, Foundations of modern analysis, Academic Press, New York, 1969.

[E] J. Eells, Jr., A setting for global analysis, Bull. Amer. Math. Soc. 72 (1966) 751-807.

[GT] D. Gilbarg and N. S. Trudinger, Elliptic partial differential equations of second order, 2nd ed., Springer-Verlag, Berlin, 1983. 
[GG] M. Golubitsky and V. Guillemin, Stable mappings and their singularities, Springer-Verlag, New York, 1973.

[GS] M. Golubitsky and D. Schaeffer, Singularities and groups in bifurcation theory, Vol. 1, Appl. Math. Sci., vol. 51, Springer-Verlag, New York, 1985.

[GSS] M. Golubitsky, I. Stewart and D. Schaeffer, Singularities and groups in bifurcation theory, Vol. 2, Appl. Math. Sci., vol. 69, Springer-Verlag, New York, 1988.

[H] P. R. Halmos, Introduction to hilbert space and the theory of spectral multiplicity, 2nd ed., Chelsea, New York, 1957.

[Ka-1] T. Kato, Perturbation theory for linear operators, Corrected printing of the 2nd ed., SpringerVerlag, New York, 1976.

[Ka-2] _ _ Schrödinger operators with singular potentials, Israel J. Math. 13 (1972), 135-148.

[Kl] V. Klee, A note on topological properties of normed-linear spaces, Proc. Amer. Math. Soc. 7 (1956), 673-674.

[Kr] M. A. Krasnosel'skii, Topological methods in the theory of nonlinear integral equations, translated by A. H. Armstrong, Macmillan, New York, 1964.

[KZ] M. A. Krasnosel'skii and P. P. Zabreiko, Geometrical methods of nonlinear analysis, translated by C. C. Fenske, Springer-Verlag, New York, 1984.

[K-1] N. H. Kuiper, The differential topology of separable Banach manifolds', Actes du Congrès International des Mathématiciens 1970, Tome 2, Gauthier-Villars, Paris, 1971, pp. 85-90.

[K-2] _ Variétés hilbertiennes; aspects géométriques, Les Presses Univ. de Montréal, Montréal, 1971.

[L] S. Lang, Differential manifolds, Addison-Wesley, Reading, Mass., 1972.

[LU] O. A. Ladyzhenskaya and N. N. Ural'tseva, Linear and quasilinear elliptic equations, translated by Scripta Technica, Academic Press, New York, 1968.

[LM] F. Lazari and A. M. Micheletti, An application of singularity theory to nonlinear differentiable mappings between Banach spaces, J. Nonlinear Anal. 11 (1987), 795-808.

[LMc] A. C. Lazer and P. J. McKenna, Multiplicity of solutions of nonlinear boundary value problems with nonlinearities crossing several higher eigenvalues, J. Reine Angew. Math. 368 (1986), 184-200.

[Ma] I. Mandhyan, Examples of global normal forms for some simple nonlinear operators, Nonlinear Anal. 13 (1989), 1057-1066.

[M] W. S. Massey, Algebraic toplogy: An introduction, Springer-Verlag, New York, 1967 (4th corrected printing, 1977).

[Mc] H. P. McKean, Singularities of a simple elliptic operator, J. Differential Geom. 25 (1987), 157-165.

[MS] H. P. McKean and J.C. Scovel, Geometry of some simple nonlinear differential operators, Ann. Scuola Norm. Sup. Pisa Cl. Sci. (4) 13 (1986), 299-346.

[P] R. Palais, Natural operations on differential forms, Trans. Amer. Math. Soc. 92 (1959), 125-141.

[R-1] B. Ruf, Singularity theory and forced secondary bifurcations, preprint, Università di Milano, 1990.

[R-2] _ Singularity theory and the geometry of a nonlinear elliptic equation, Ann. Sci. Norm. Sup. Pisa Cl. Sci. (4) 17 (1990), 1-33.

[Sc] L. Schwartz, Théorie des distributions, Tome 1, Hermann, Paris, 1950.

[So] S. L. Sobolev, Applications of functional analysis in mathematical physics, Leningrad, 1950, translated by F. Browder, Amer. Math. Soc., Providence, RI, 1963.

[Sp] E. H. Spanier, Algebraic topology, McGraw-Hill, New York, 1966.

[Tr] F. Treves, Basic linear partial differential equations, Academic Press, New York, 1975.

[T] N.S. Trudinger, Linear elliptic operators with measurable coefficients, Ann. Scuola Norm. Sup. Pisa (3) 27 (1973), 265-308.

[W] S. Willard, General topology, Addison-Wesley, Reading, Mass., 1970. 
[WP] A. E. R. Woodcock and T. Poston, A geometrical study of the elementary catastrophes, Lecture Notes in Math., vol. 377, Springer-Verlag, Berlin, 1974.

[Z] E. Zeidler, Nonlinear functional analysis. I. Fixed-point theorems, Springer-Verlag, New York, 1986.

Department of Mathematics, Syracuse University, Syracuse New York 13244-1150

E-mail address: ptchurch@suvm.syr.edu

Department of Mathematics, University of New England, Armidale, N.S.W. 2351, AUSTRALIA

Department of Mathematics, University of Alberta, Edmonton, Alberta, Canada T6G 2G1

E-mail address: usertimo@mts.ucs.ualberta.ca 